\title{
Órbitas de Sussmann e aplicações
}



SERVIÇO DE PÓS-GRADUAÇÃO DO ICMC-USP

Data de Depósito:

Assinatura:

\title{
Órbitas de Sussmann e aplicações
}

\author{
Renato Andrielli Laguna
}

Orientador: Prof. Dr. Sérgio Luís Zani

Dissertação apresentada ao Instituto de Ciências Matemáticas e de Computação - ICMC-USP, como parte dos requisitos para obtenção do título de Mestre em Ciências - Matemática. VERSÃO REVISADA.

USP - São Carlos

Junho/2011 
Ficha catalográfica elaborada pela Biblioteca Prof. Achille Bassi e Seção Técnica de Informática, ICMC/USP, com os dados fornecidos pelo(a) autor(a)

Laguna, Renato Andrielli
Órbitas de Sussmann e aplicações / Renato Andrielli
Laguna; orientador Sérgio Luís Zani -- São Carlos,
2011. $97 \mathrm{p.}$
Dissertação (Mestrado - Programa de Pós-Graduação en
-- Instituto de Ciências Matemáticas e de
Computação, Universidade de São Paulo, 2011.
1. EQUAÇõES DIFERENCIAIS PARCIAIS LINEARES. 2.
CAMPOS VETORIAIS. 3. ÓRBITAS DE SUSSMANN. 4.
RESOLUBILIDADE LOCAL. I. Zani, Sérgio Luís, orient.
II. Título.




\section{Resumo}

Nesta dissertação, estudamos as órbitas de uma família $D$ de campos vetoriais suaves em uma variedade suave $M$. O objetivo é demonstrar dois teoremas de Sussmann: o primeiro teorema diz que as órbitas são subvariedades integrais de uma certa distribuição $P_{D}$ de vetores tangentes em $M$. O segundo teorema dá condições necessárias e suficientes para que $P_{D}$ seja igual à distribuição gerada pelos campos de $D$. Como aplicação, estudamos uma caracterização da condição $(\mathcal{P})$ de Nirenberg-Treves para campos vetoriais complexos em $\mathbb{R}^{2}$. 



\begin{abstract}
In this dissertation, we study the orbits of a family $D$ of smooth vector fields on a smooth manifold $M$. The goal is to demonstrate two theorems of Sussmann: the first theorem says that the orbits are integral submanifolds of a certain distribution $P_{D}$ of tangent vectors of $M$. The second theorem gives necessary and sufficient conditions for $P_{D}$ to be the same as the distribution generated by the vector fields of $D$. As an application, we study a characterization of the condition $(\mathcal{P})$ of Nirenberg and Treves for complex vector fields on $\mathbb{R}^{2}$.
\end{abstract}





\section{Sumário}

1 Conceitos Fundamentais $\quad 7$

1.1 Campos vetoriais complexos . . . . . . . . . 7

1.1.1 Definição . . . . . . . . . . . . 7

1.1.2 Vetores tangentes complexos ........... 8

1.1.3 Restrição de campo vetorial a um aberto . . . . . . . . 9

1.1.4 Forma local dos campos vetoriais . . . . . . . . 10

1.2 Derivada de uma curva . . . . . . . . . . . . . . 12

1.3 Diferencial . . . . . . . . . . . . . . . 13

1.3.1 Derivadas parciais de uma aplicação . . . . . . . . 15

1.3.2 Matriz jacobiana . . . . . . . . . . . . 15

1.4 Teoremas da imersão, da submersão e do posto . . . . . . . . . 16

1.5 Subvariedades imersas . . . . . . . . . . . . 17

1.5.1 Unicidade .................. 20

1.5.2 União de subvariedades imersas . . . . . . . . . . . 24

1.5.3 Restrição de campo vetorial a subvariedade . . . . . . 25

1.5.4 Imagem de subvariedade por difeomorfismo . . . . . . 28

2 Órbitas de Sussmann $\quad 29$

2.1 Curvas integrais e fluxo . . . . . . . . . . . . . . . 29

2.1.1 Curvas integrais . . . . . . . . . . . . 29

2.1.2 Fluxo ........................ 31

2.2 Difeomorfismos locais . . . . . . . . . . . . . . . . . . . . 32

2.2.1 Definição e operações . . . . . . . . . . . . . . . . . . 32

2.2 .2 As aplicações $X_{t} \ldots \ldots \ldots \ldots . \ldots . \ldots 34$

2.2.3 Grupos de difeomorfismos locais . . . . . . . . . 34

2.3 Famílias de campos vetoriais e órbitas . . . . . . . . . . . . 35

2.3.1 Famílias de campos vetoriais . . . . . . . . . . 35

2.3.2 Definição das $D$-órbitas . . . . . . . . . 36

2.3.3 Definição por curvas integrais por partes . . . . . 36

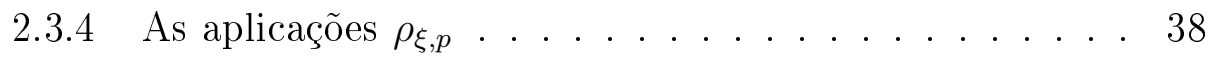

2.3.5 Topologia da $D$-órbita . . . . . . . . . . . 39 
2.4 Distribuições . . . . . . . . . . . . . . . . . . 40

2.4.1 Subvariedades integrais . . . . . . . . . . . . . 41

$2.4 .2 \quad D$-invariância . . . . . . . . . . . . 43

2.4.3 Posto das $D$-órbitas . . . . . . . . . . . . . . 45

2.4 .4 Involutividade . . . . . . . . . . . . . 46

2.5 Teoremas principais . . . . . . . . . . . . . . . . 48

2.5.1 Que as $D$-órbitas são subvariedades . . . . . . . 48

2.5.2 Condições para que $\Delta_{D}=P_{D} \ldots \ldots \ldots . \ldots 5$

3 Um Parêntese: Transformada de Fourier 63

3.1 Aproximação da identidade . . . . . . . . . . . . . . 63

3.2 Transformada de Fourier em $L^{1}(\mathbb{R}) \ldots \ldots$. . . . . . . 66

3.2.1 Funções de Schwartz . . . . . . . . . . . . . . . . 72

3.2.2 Transformada de Fourier inversa . . . . . . . . . 75

3.2.3 Aplicação: o núcleo de Poisson no semiplano . . . . . . 77

4 Sobre a Condição $(\mathcal{P})$ em $\mathbb{R}^{2} \quad 79$

4.1 Resolubilidade Local . . . . . . . . . . . . . . . . 79

4.2 Uma primeira definição da condição $(\mathcal{P}) \ldots \ldots . . . . . .83$

4.3 Uma segunda definição da condição $(\mathcal{P}) \ldots . . . . .84$

4.3.1 Parêntese: produto exterior de campos vetoriais . . . . 84

4.3.2 A definição em questão . . . . . . . . . . . . 85

4.4 Resolubilidade local em $L^{2} \ldots \ldots \ldots$. . . . . . . 87 


\section{Introdução}

Sejam $M$ uma variedade suave e $X$ um campo vetorial suave em $M$. Uma curva integral maximal de $X$ pode ser apenas de três tipos:

(i) uma constante $\alpha: t \in \mathbb{R} \mapsto p_{0} \in M$;

(ii) uma curva regular sem auto-intersecção;

(iii) uma curva regular $\alpha: \mathbb{R} \rightarrow M$ que é periódica e só se intercepta no fim de seu período, isto é, existe $T>0$ tal que, para todos $t, s \in \mathbb{R}$,

$$
\alpha(t)=\alpha(s) \Leftrightarrow t-s \in T \mathbb{Z} .
$$

Nos três casos, a imagem de $\alpha$ é uma subvariedade imersa de $M$. Note que esta subvariedade nem sempre é mergulhada - um exemplo é o chamado fluxo irracional no toro (ver Sotomayor [7], capítulo VI, seção 7).

Para cada $p \in M$, seja $S_{p}$ a imagem de uma curva integral maximal de $X$ que passa por $p$. $S_{p}$ é bem-definida, pois a curva integral maximal de $X$ passando por $p$ é única a menos de reparametrização.

Isto particiona $M$ em subvariedades disjuntas (quando não são iguais) $S_{p}$, cujos espaços tangentes em cada ponto coincidem com o espaço gerado por $X$ e que são conexas e maximais com relação a esta propriedade. As $S_{p}$ são as órbitas do campo vetorial $X$.

Agora, vamos introduzir um segundo campo $Y \in \mathfrak{X}(M)$. Será possível particionar $M$ em subvariedades $S_{p}$ conexas de forma que o espaço tangente de cada uma (em cada ponto) coincida com o espaço gerado por $X$ e $Y$ e que elas sejam maximais ?

A resposta vai depender da escolha de $X$ e $Y$. Por exemplo, se o colchete de Lie $[X, Y]$ for linearmente independente (em algum ponto) de $X$ e $Y$, a resposta é "não".

Caso a resposta seja "sim", cada $S_{p}$ deverá conter as curvas integrais de $X$ ou de $Y$ que a interceptam.

Isto nos leva a considerar como candidato a $S_{p}$ o menor conjunto (contendo $p$ ) que contém todas as curvas integrais de $X$ ou de $Y$ que o interceptam. Tal conjunto é uma órbita do par de campos $\{X, Y\}$. 
Mais geralmente, seja $M$ uma variedade suave e seja $D$ um conjunto de campos vetoriais suaves definidos em abertos de $M$, de forma que todo ponto $p \in M$ está no domínio de pelo menos um campo de $D$. As $D$-órbitas são as classes de equivalência da seguinte relação de equivalência: dois pontos $p$ e $q$ são equivalentes se podem ser ligados por uma sequência finita de segmentos de curvas integrais de campos de $D$.

Uma distribuição é uma aplicação $\Delta$ que associa a cada ponto $p$ de $M$ um subespaço vetorial $\Delta(p)$ de $\mathrm{T}_{p} M$. A distribuição que associa a cada ponto o espaço gerado pelos campos de $D$ naquele ponto é denotada por $\Delta_{D}$.

Sussmann [10] demonstra dois teoremas principais: o primeiro teorema diz que as $D$-órbitas, dotadas de uma topologia apropriada, são subvariedades imersas de $M$ e que seus espaços tangentes coincidem, em cada ponto, com uma certa distribuição $P_{D}$ (definida no artigo) que é involutiva e contém $\Delta_{D}$; o segundo teorema dá condições necessárias e suficientes para que $P_{D}$ seja igual a $\Delta_{D}$.

Nesta dissertação, demonstramos em detalhes os dois teoremas supracitados. Em seguida, faremos uma breve discussão da condição $(\mathcal{P})$ de Nirenberg-Treves em $\mathbb{R}^{2}$.

O primeiro capítulo contém as definições de campo vetorial e do diferencial de uma aplicação entre variedades, conforme são usadas neste trabalho, junto com algumas propriedades importantes das subvariedades imersas. Por variedade, entende-se um espaço topológico de Hausdorff dotado de uma estrutura diferenciável $\mathcal{C}^{\infty}$. As definições de campo vetorial complexo e vetores tangentes complexos usadas neste capítulo seguem o capítulo I de Berhanu, Cordaro e Hounie [1].

O segundo capítulo é uma exposição detalhada das definições e teoremas principais de [10]. Duas modificações relevantes foram feitas:

(i) No lema 2.72, que é o lema 5.3 de [10], a demonstração de que a subvariedade em questão é um aberto na topologia da órbita foi feita de outro jeito, devido a um "from this it follows easily that" do qual, aparentemente, não seguia o resultado desejado. A nova demonstração é mais longa e desagradável.

(ii) No teorema 2.80, que é o teorema 4.2 de [10], foi adicionado à condição (e) o requisito de que a distribuição $\Delta$ tenha posto constante nas $D$-órbitas. Tal modificação é necessária para evitar que a condição (e) se aplique ao exemplo 2.68, o que tornaria o teorema falso. A origem do erro está no uso de um lema de Lobry [4] que é falso (ver [5]). A condição (e') da errata de Sussmann aparentemente tem o mesmo problema. 
O terceiro capítulo é uma introdução à transformada de Fourier na reta real. Isto é necessário para os cálculos do quarto capítulo.

O quarto capítulo, seguindo as ideias do início do capítulo IV de [1], é sobre a condição $(\mathcal{P})$ para a resolubilidade local de um campo vetorial complexo não-singular $L$ em $\mathbb{R}^{2}$. Localmente, a equação $L u=f$ sempre pode ser transformada (via mudança de coordenadas) em

$$
\frac{\partial u}{\partial t}+i b \frac{\partial u}{\partial x}=f
$$

onde $b$ é uma função que só assume valores reais. Para campos que já são da forma $L=\partial_{t}+i b \partial_{x}$, com $b$ real, definimos a condição $(\mathcal{P})$ em um ponto $p$ como sendo: que $b$ não muda de sinal sobre as retas verticais (que são as de coordenada $x$ constante) dentro de algum retângulo que contém $p$. Uma segunda definição da condição $(\mathcal{P})$ é dada, independente de coordenadas, que faz uso das órbitas de Sussmann e que é equivalente à definição anterior quando aquela se aplica. Concluímos mostrando que a condição $(\mathcal{P})$ implica a resolubilidade local em $L^{2}$.

\section{Agradecimentos}

(i) Ao meu orientador, Sérgio Luís Zani.

(ii) Ao professor Paulo Leandro Dattori da Silva, que me emprestou o seu livro de variedades e me ajudou com algumas dúvidas.

(iii) Mais geralmente, aos professores e funcionários do Instituto de Ciências Matemáticas e de Computação.

(iv) Aos meus colegas da pós-graduação, pessoas tolerantes e amigáveis.

(v) A Deus e à minha família, por motivos óbvios.

(vi) Ao Brasil.

(vii) Em particular, ao CNPq, que financiou este trabalho. 


\section{Capítulo 1}

\section{Conceitos Fundamentais}

\subsection{Campos vetoriais complexos}

\subsubsection{Definição}

Definição 1.1. (i) Um campo vetorial complexo na variedade $M$ é uma aplicação $X: \mathcal{C}^{\infty}(M) \rightarrow \mathcal{C}^{\infty}(M)$ satisfazendo as seguintes propriedades:

$\mathbb{C}$-Linearidade $\forall \lambda \in \mathbb{C}, f, g \in \mathcal{C}^{\infty}(M)$ :

$$
X(\lambda f+g)=\lambda X f+X g .
$$

Regra de Leibniz $\forall f, g \in \mathcal{C}^{\infty}(M)$ :

$$
X(f g)=(X f) g+(X g) f .
$$

(ii) Um campo vetorial complexo $X$ é real (neste caso, chamado somente de campo vetorial) quando, sempre que $f \in \mathcal{C}^{\infty}(M)$ for uma função que assume somente valores reais, $X f$ somente assumir valores reais.

Observação 1.2. $\mathcal{C}^{\infty}(M)$ denota o conjunto de todas as funções $f: M \rightarrow$ $\mathbb{C}$ que são suaves. Denotaremos o conjunto de todos os campos vetoriais complexos em $M$ por $\mathbb{C} \mathfrak{X}(M)$ e o conjunto de todos os campos vetoriais (reais) em $M$ por $\mathfrak{X}(M)$.

Proposição 1.3. Se $f$ é constante, $X f=0$

Demonstração. Seja $f$ a função de valor constante $\alpha . X\left(f^{2}\right)=2(\alpha X f)=$ $\alpha X f$, pela regra de Leibniz e pela $\mathbb{C}$-linearidade (respectivamente). Então $\alpha=0$ ou $X f=0$. Se $\alpha=0, X f=X(0 f)=0(X f)=0$, pela $\mathbb{C}$-linearidade. 
Proposição 1.4. Sejam $f, g \in \mathcal{C}^{\infty}(M)$ tais que $f=g$ em um aberto $U \in M$. Então $X f=X g$ em $U$.

Demonstração. Dado $p \in U$, mostremos que $X f(p)=X g(p)$. Defina $\psi_{p} \in$ $\mathcal{C}^{\infty}(M)$ tal que $\psi_{p}(p)=0$ e $\psi_{p}=1$ fora do aberto U. Então $f-g=\left(\psi_{p}\right)(f-g)$. Pela regra de Leibniz:

$$
\begin{array}{r}
X(f-g)=X\left(\left(\psi_{p}\right)(f-g)\right)=\left(X \psi_{p}\right)(f-g)+(X(f-g)) \psi_{p} \\
\therefore X(f-g)(p)=0 \quad \therefore X f(p)=X g(p) .
\end{array}
$$

\subsubsection{Vetores tangentes complexos}

Definição 1.5. (i) Um vetor tangente complexo no ponto $p \in M$ é uma aplicação $\mathbb{C}$-linear $\vec{v}_{p}: \mathcal{C}^{\infty}(p) \rightarrow \mathbb{C}$ satisfazendo a seguinte regra de Leibniz:

$$
\left.\forall f\right|_{p},\left.g\right|_{p} \in \mathcal{C}^{\infty}(p): \vec{v}_{p}\left[\left.\left.f\right|_{p} g\right|_{p}\right]=\vec{v}_{p}\left[\left.f\right|_{p}\right] g(p)+\vec{v}_{p}\left[\left.g\right|_{p}\right] f(p) .
$$

(ii) Um vetor tangente complexo $\vec{v}_{p}$ é real (neste caso, chamado simplesmente de vetor tangente) quando, sempre que $\left.f\right|_{p} \in \mathcal{C}^{\infty}(p)$ for um germe de função real, $\vec{v}_{p}\left[\left.f\right|_{p}\right]$ é real.

(iii) O conjunto de todos os vetores tangentes reais de $M$ em $p$ é denotado por $\mathrm{T}_{p} M$ e chamado de espaço tangente de $M$ em $p$.

(iv) O conjunto de todos os vetores tangentes complexos de $M$ em $p$ é denotado por $\mathbb{C T}_{p} M$ e chamado de espaço tangente complexo de $M$ em $p$.

(v) A união $\bigcup_{p \in M} \mathrm{~T}_{p} M$ é chamada de fibrado tangente de $M$ e denotada por TM.

(vi) A união $\bigcup_{p \in M} \mathbb{C T}_{p} M$ é chamada de fibrado tangente complexificado de $M$ e denotada por $\mathbb{C T} M$.

(vii) Se $X \in \mathbb{C} \mathfrak{X}(M)$ é um campo vetorial complexo em $M$ e $p \in M$, o vetor tangente complexo $\left.X\right|_{p}$ é definido por

$$
\left.X\right|_{p}\left[\left.f\right|_{p}\right]=X f(p) .
$$

Observação 1.6. Denotamos por $\mathcal{C}^{\infty}(p)$ a $\mathbb{C}$-álgebra dos germes de função suave em $p \in M .\left.f\right|_{p}$ denota o germe de uma função $f$ no ponto $p$. 
Observação 1.7. Usaremos colchetes $\left(\vec{v}_{p}\left[\left.f\right|_{p}\right]\right)$ para denotar a aplicação de um vetor tangente complexo a um germe e usaremos parênteses, ou mera concatenação $(X(f)$ ou $X f)$ para denotar a aplicação do campo vetorial complexo a uma função.

Proposição 1.8. Seja $\phi: M \rightarrow \mathbb{C T} M$ uma função que associa a cada ponto $p$ da variedade $M$ um vetor tangente complexo $\phi(p) \in \mathbb{C T}_{p} M$. Se, para cada $f \in \mathcal{C}^{\infty}(M)$, a função $\phi_{f}: p \mapsto \phi(p)\left[\left.f\right|_{p}\right]$ é suave, então existe um único campo vetorial complexo $X$ tal que $\left.X\right|_{p}=\phi(p), \quad \forall p \in M$.

Demonstração. Defina $X$ pela fórmula $X f=\phi_{f}$. A $\mathbb{C}$-linearidade de $X$ e a regra de Leibniz serão consequência das propriedades dos vetores tangentes complexos.

Dado $p \in M,\left.X\right|_{p}\left[\left.f\right|_{p}\right]=X f(p)=\phi_{f}(p)=\phi(p)\left[\left.f\right|_{p}\right], \forall f \in \mathcal{C}^{\infty}(M)$. Portanto, $\left.X\right|_{p}=\phi(p)$.

Mostremos a unicidade de $X$.

Sejam $X^{1}$ e $X^{2}$ campos vetoriais complexos com $\left.X^{1}\right|_{p}=\left.X^{2}\right|_{p}=\phi(p)$ para todo $p \in M$. Então, dado $f \in \mathcal{C}^{\infty}(M)$,

$$
X^{1} f(p)=\left.X^{1}\right|_{p}\left[\left.f\right|_{p}\right]=\phi(p)\left[\left.f\right|_{p}\right]=\left.X^{2}\right|_{p}\left[\left.f\right|_{p}\right]=X^{2} f(p), \quad \forall p \in M .
$$

$\therefore X^{1} f=X^{2} f$, para toda $f \in \mathcal{C}^{\infty}(M)$. Portanto $X^{1}=X^{2}$.

\subsubsection{Restrição de campo vetorial a um aberto}

Proposição 1.9. Seja $X$ um campo vetorial complexo na variedade $M e$ $U \subset M$ um aberto. Então existe um único campo vetorial complexo $\left.X\right|_{U} \in$ $\mathbb{C} \mathfrak{X}(U)$ tal que $\left.X\right|_{U}\left(\left.f\right|_{U}\right)=\left.(X f)\right|_{U}$, para toda $f \in \mathcal{C}^{\infty}(M)$.

Demonstração. Dado $p \in U$, o vetor tangente complexo $\left.X\right|_{p}$ está em $\mathbb{C T}_{p} M$. Queremos identificar $\mathbb{C T}_{p} M$ com $\mathbb{C T}_{p} U$. Se $f \in \mathcal{C}^{\infty}(M)$, identificamos o germe $\left.f\right|_{p}$ (que é germe na variedade $M$ ) com o germe $\left.\left(\left.f\right|_{U}\right)\right|_{p}$ (germe em $U$ ). É fácil ver que tal identificação é um isomorfismo de álgebra entre $\mathcal{C}^{\infty}(p)_{M}$ e $\mathcal{C}^{\infty}(p)_{U}$. Assim, vamos tratar estes espaços de germes como se fossem o mesmo.

Identificando-se os germes, ficam identificados os vetores tangentes complexos de $U$ e $M$.

Definimos $\left.X\right|_{U}$, usando a proposição 1.8, por $\left.\left(\left.X\right|_{U}\right)\right|_{p}=\left.X\right|_{p}, \forall p \in U$. Para isso, devemos mostrar que as aplicações $\phi_{f}:\left.p \in U \mapsto X\right|_{p}(f)$, definidas para toda $f \in \mathcal{C}^{\infty}(U)$, são suaves.

Mostremos que $\phi_{f}$ é localmente suave em um ponto $p_{0} \in U$ qualquer. De fato, pois $\phi_{f}$ é igual, em alguma vizinhança de $p_{0}$, a $X g$, onde $g \in \mathcal{C}^{\infty}(M)$ é uma função com $\left.f\right|_{p_{0}}=\left.g\right|_{p_{0}}$. 
Assim definido $\left.X\right|_{U}$, usando a identificação entre germes de $M$ e $U$ que fizemos, temos que

$$
\left.X\right|_{U}\left(\left.f\right|_{U}\right)(p)=\left.\left(\left.X\right|_{U}\right)\right|_{p}\left[\left.\left(\left.f\right|_{U}\right)\right|_{p}\right]=\left.X\right|_{p}\left[\left.f\right|_{p}\right]=X f(p)
$$

para toda $f \in \mathcal{C}^{\infty}(M)$ e todo $p \in U$.

Observação 1.10. O aberto $U \subset M$ é considerado uma variedade, cuja estrutura diferenciável é obtida pela restrição da estrutura diferenciável de $M$.

\subsubsection{Forma local dos campos vetoriais}

Definição 1.11. Seja $M$ uma variedade e $\vec{x}: U \subset M \rightarrow V \subset \mathbb{R}^{m}$ uma carta local de $M$. Seja $f \in \mathcal{C}^{\infty}(U)$. Podemos escrever $f=f_{\vec{x}} \circ \vec{x}$, onde $f_{\vec{x}}: V \rightarrow \mathbb{C}$ é a função suave $f \circ \vec{x}^{-1}$. Então definimos as derivadas parciais

$$
\frac{\partial f}{\partial x_{j}} \in \mathcal{C}^{\infty}(U), j=1, \ldots, m
$$

pela fórmula:

$$
\frac{\partial f}{\partial x_{j}}=\frac{\partial}{\partial x_{j}} f=\partial_{j}\left(f_{\vec{x}}\right) \circ \vec{x} .
$$

As aplicações

$$
\frac{\partial}{\partial x_{j}}: \mathcal{C}^{\infty}(U) \longrightarrow \mathcal{C}^{\infty}(U)
$$

(que também podem ser denotadas $\partial_{x_{j}}$, quando quisermos economizar espaço) são campos vetoriais reais em $U$. Dada $g \in \mathcal{C}^{\infty}(M)$, fica denotado

$$
\frac{\partial g}{\partial x_{j}}=\frac{\partial}{\partial x_{j}}\left(\left.g\right|_{U}\right)
$$

Observação 1.12. O símbolo $\partial_{j}$ denota a derivada parcial usual na $j$-ésima coordenada.

Proposição 1.13. Seja $X \in \mathbb{C} \mathfrak{X}(U)$, onde $U$ é o dominio de uma carta local $\vec{x}: U \subset M \rightarrow V \subset \mathbb{R}^{m}$ da variedade $M$. Então

$$
X=\sum_{j=1}^{m} X\left(x_{j}\right) \frac{\partial}{\partial x_{j}} .
$$


Demonstração. Sejam $f \in \mathcal{C}^{\infty}(U)$ e $p \in U$. Seja $r>0$ tal que a bola aberta $B(\vec{x}(p), r)$ está contida em $V$. Então $\vec{x}-\vec{x}(p)$ é uma carta local definida em $U$ que se anula em $p$. Restringindo esta carta, obtemos uma carta local $\vec{y}: U^{\prime} \rightarrow B(0, r), \operatorname{com} \vec{y}(p)=0$.

Seja $f_{\vec{y}}=\left.f\right|_{U^{\prime}} \circ \vec{y}^{-1}: B(0, r) \rightarrow \mathbb{C}$ a expressão de $f$ nas coordenadas locais de $\vec{y}$. Dado $h \in B(0, r)$, seja $\alpha_{h}:(0,1) \rightarrow B(0, r)$ definida por $\alpha_{h}(t)=t h$. Pelo teorema fundamental do cálculo e pela regra da cadeia,

$$
\begin{aligned}
f_{\vec{y}}(h) & =f_{\vec{y}}(0)+\int_{0}^{1}\left(\frac{\mathrm{d}}{\mathrm{d} t} f_{\vec{y}}\left(\alpha_{h}(t)\right)\right) \mathrm{d} t=\int_{0}^{1}\left\langle\nabla f_{\vec{y}}\left(\alpha_{h}(t)\right), \alpha_{h}^{\prime}(t)\right\rangle \mathrm{d} t \\
& =\int_{0}^{1} \sum_{j=1}^{m} h_{j} \partial_{j}\left(f_{\vec{y}}\right)(h t) \mathrm{d} t=\sum_{j=1}^{m} h_{j} \int_{0}^{1} \partial_{j}\left(f_{\vec{y}}\right)(h t) \mathrm{d} t .
\end{aligned}
$$

Defina as funções

$$
g_{j}(q)=\int_{0}^{1} \partial_{j}\left(f_{\vec{y}}\right)(t \vec{y}(q)) \mathrm{d} t, \quad j=1, \ldots, m,
$$

$\operatorname{com} q \in U^{\prime}$. Note que $g_{j}(p)=\partial f / \partial y_{j}(p)$. Temos:

$$
\left.f\right|_{U^{\prime}}=f(p)+\sum_{j=1}^{m} y_{j} g_{j}
$$

Aplicando o campo $\left.X\right|_{U^{\prime}}$ e usando a regra de Leibniz, obtemos:

$$
\begin{aligned}
X f(p) & =\sum_{j=1}^{m}(\left.X\right|_{U^{\prime}}\left(y_{j}\right)(p) g_{j}(p)+\left.\underbrace{y_{j}(p)}_{=0} X\right|_{U^{\prime}}\left(g_{j}\right)(p))=\left.\sum_{j=1}^{m} X\right|_{U^{\prime}}\left(y_{j}\right)(p) g_{j}(p) \\
& =\left.\sum_{j=1}^{m} X\right|_{p}\left[\left.y_{j}\right|_{p}\right] \frac{\partial f}{\partial y_{j}}(p)=\sum_{j=1}^{m} X\left(x_{j}\right)(p) \frac{\partial f}{\partial x_{j}}(p) .
\end{aligned}
$$

Observação 1.14. O mesmo procedimento que foi usado na proposição acima serve para demonstrar que todo vetor tangente complexo $\vec{v}_{p} \in \mathbb{C T}_{p} M$ se decompõe em coordenadas locais na forma

$$
\vec{v}_{p}=\left.\sum_{j=1}^{m} \vec{v}_{p}\left[x_{j}\right] \frac{\partial}{\partial x_{j}}\right|_{p}
$$




\subsection{Derivada de uma curva}

Definição 1.15. Seja $\alpha:(a, b) \rightarrow M$ uma curva suave em $M$. Para cada $t \in(a, b)$, definimos $\alpha^{\prime}(t) \in \mathrm{T}_{p} M$ (que também pode ser denotado por $\frac{\mathrm{d}}{\mathrm{d} t} \alpha(t)$ ) pela equação:

$$
\alpha^{\prime}(t)\left[\left.f\right|_{\alpha(t)}\right]:=(f \circ \alpha)^{\prime}(t)
$$

Verifiquemos que $\alpha^{\prime}(t): \mathcal{C}^{\infty}(\alpha(t)) \rightarrow \mathbb{C}$ satisfaz a definição de um vetor tangente complexo:

$$
\begin{aligned}
\text { C-linearidade } & \alpha^{\prime}(t)\left[\left.f\right|_{\alpha(t)}+\lambda\left(\left.g\right|_{\alpha(t)}\right)\right]= \\
= & \alpha^{\prime}(t)\left[\left.(f+\lambda g)\right|_{\alpha(t)}\right]=((f+\lambda g) \circ \alpha)^{\prime}(t) \\
= & ((f \circ \alpha)+\lambda(g \circ \alpha))^{\prime}(t)=\alpha^{\prime}(t)\left[\left.f\right|_{\alpha(t)}\right]+\lambda \alpha^{\prime}(t)\left[\left.g\right|_{\alpha(t)}\right] .
\end{aligned}
$$

Regra de Leibniz $\alpha^{\prime}(t)\left[\left.\left.f\right|_{\alpha(t)} g\right|_{\alpha(t)}\right]=\alpha^{\prime}(t)\left[\left.(f g)\right|_{\alpha(t)}\right]$

$$
\begin{aligned}
& =(f g \circ \alpha)^{\prime}(t)=\frac{\mathrm{d}}{\mathrm{d} t} f(\alpha(t)) g(\alpha(t)) \\
& =(f \circ \alpha)^{\prime}(t)(g \circ \alpha)(t)+(f \circ \alpha)(t)(g \circ \alpha)^{\prime}(t) \\
& =\alpha^{\prime}(t)\left[\left.f\right|_{\alpha(t)}\right] g(\alpha(t))+f(\alpha(t)) \alpha^{\prime}(t)\left[\left.g\right|_{\alpha(t)}\right] .
\end{aligned}
$$

Como $(f \circ \alpha)^{\prime}(t)$ é real sempre que $f$ é uma função real, $\alpha^{\prime}(t)$ é um vetor tangente real.

Exemplo 1.16 (Fórmula local da derivada). Seja $\vec{x}=\left(x_{1}, \ldots, x_{m}\right): U \subset$ $M \rightarrow V \subset \mathbb{R}^{m}$ uma carta local de $M$ tal que $\alpha\left(t_{0}\right) \in U$ para algum $t_{0}$. Seja $\delta>0$ tal que $\alpha\left(\left(t_{0}-\delta, t_{0}+\delta\right)\right) \subset U$. Seja $\tilde{\alpha}=\left.\alpha\right|_{\left(t_{0}-\delta, t_{0}+\delta\right) \rightarrow U}$ (restrição de domínio e contra-domínio de $\alpha$ ). Defina $\alpha^{j}=x_{j} \circ \tilde{\alpha}, \forall j=1 \ldots, m$. Assim, a curva $\alpha$ é dada, para $\left|t-t_{0}\right|<\delta$, pelas suas coordenadas

$$
\vec{x}(\alpha(t))=\left(\alpha^{1}(t), \ldots, \alpha^{m}(t)\right) .
$$

Dado um germe de função suave $\left.f\right|_{\alpha\left(t_{0}\right)}$, calculemos $\alpha^{\prime}\left(t_{0}\right)\left[\left.f\right|_{\alpha\left(t_{0}\right)}\right]$ :

$$
\begin{aligned}
\alpha^{\prime}\left(t_{0}\right)\left[\left.f\right|_{\alpha\left(t_{0}\right)}\right] & =(f \circ \alpha)^{\prime}\left(t_{0}\right)=(f \circ \tilde{\alpha})^{\prime}\left(t_{0}\right)=\left(f \circ(\vec{x})^{-1} \circ \vec{x} \circ \tilde{\alpha}\right)^{\prime}\left(t_{0}\right) \\
& =\sum_{j=1}^{n}\left(x_{j} \circ \tilde{\alpha}\right)^{\prime}\left(t_{0}\right) \partial_{j}\left(f \circ(\vec{x})^{-1}\right)\left(\vec{x} \circ \tilde{\alpha}\left(t_{0}\right)\right) \quad \text { (regra da cadeia) } \\
& =\left.\sum_{j=1}^{n}\left(\alpha^{j}\right)^{\prime}\left(t_{0}\right) \frac{\partial}{\partial x_{j}}\right|_{\tilde{\alpha}\left(t_{0}\right)}\left[\left.f\right|_{\tilde{\alpha}\left(t_{0}\right)}\right] .
\end{aligned}
$$


Assim, a derivada de uma curva em coordenadas locais é dada pela fórmula:

$$
\alpha^{\prime}(t)=\left.\sum_{j=1}^{n}\left(\alpha^{j}\right)^{\prime}\left(t_{0}\right) \frac{\partial}{\partial x_{j}}\right|_{\alpha\left(t_{0}\right)}
$$

\subsection{Diferencial}

Definição 1.17. Seja $F: M \rightarrow N$ uma aplicação suave entre variedades. Se $g \in \mathcal{C}^{\infty}(N), g \circ F \in \mathcal{C}^{\infty}(M)$. Dado um vetor tangente complexo $\vec{v}_{p}$ em $\mathbb{C T}_{p} M$, definimos um vetor tangente complexo em $\mathbb{C T}_{F(p)} N$, que será chamado de $\mathrm{d} F\left(\vec{v}_{p}\right)$, pela seguinte fórmula:

$$
\mathrm{d} F\left(\vec{v}_{p}\right)\left[\left.g\right|_{F(p)}\right]=\vec{v}_{p}\left[\left.(g \circ F)\right|_{p}\right] .
$$

Se $\vec{v}_{p}$ for real, $\mathrm{d} F\left(\vec{v}_{p}\right)$ será real e com isto definimos o diferencial de $F$ como a aplicação

$$
\mathrm{d} F: \mathrm{T} M \longrightarrow \mathrm{T} N .
$$

Se considerarmos $p \in M$ fixado, a restrição

$$
\left.\mathrm{d} F\right|_{p}: \mathrm{T}_{p} M \longrightarrow \mathrm{T}_{F(p)} N
$$

é uma aplicação linear cujo posto é chamado posto de $F$ em p.

Observação 1.18. Verifiquemos que $\mathrm{d} F\left(\vec{v}_{p}\right)$ satisfaz os requisitos de um vetor tangente complexo:

C-Linearidade $\mathrm{d} F\left(\vec{v}_{p}\right)\left[\left.g_{1}\right|_{F(p)}+\lambda\left(\left.g_{2}\right|_{F(p)}\right)\right]$

$$
\begin{aligned}
& =\mathrm{d} F\left(\vec{v}_{p}\right)\left[\left.\left(g_{1}+\lambda g_{2}\right)\right|_{F(p)}\right]=\vec{v}_{p}\left[\left.\left(\left(g_{1}+\lambda g_{2}\right) \circ F\right)\right|_{p}\right] \\
& =\vec{v}_{p}\left[\left.\left(g_{1} \circ F+\lambda\left(g_{2} \circ F\right)\right)\right|_{p}\right]=\vec{v}_{p}\left[\left.\left(g_{1} \circ F\right)\right|_{p}+\lambda\left(\left.\left(g_{2} \circ F\right)\right|_{p}\right)\right] \\
& =\vec{v}_{p}\left[\left.\left(g_{1} \circ F\right)\right|_{p}\right]+\lambda \vec{v}_{p}\left[\left.\left(g_{2} \circ F\right)\right|_{p}\right] \\
& =\mathrm{d} F\left(\vec{v}_{p}\right)\left[\left.g_{1}\right|_{F(p)}\right]+\lambda \mathrm{d} F\left(\vec{v}_{p}\right)\left[\left.g_{2}\right|_{F(p)}\right] .
\end{aligned}
$$

Regra de Leibniz $\mathrm{d} F\left(\vec{v}_{p}\right)\left[\left.\left.g_{1}\right|_{F(p)} g_{2}\right|_{F(p)}\right]=$

$$
\begin{aligned}
& =\mathrm{d} F\left(\vec{v}_{p}\right)\left[\left.\left(g_{1} g_{2}\right)\right|_{F(p)}\right]=\vec{v}_{p}\left[\left.\left(\left(g_{1} g_{2}\right) \circ F\right)\right|_{p}\right]=\vec{v}_{p}\left[\left.\left.\left(g_{1} \circ F\right)\right|_{p}\left(g_{2} \circ F\right)\right|_{p}\right] \\
& =\vec{v}_{p}\left[\left.\left(g_{1} \circ F\right)\right|_{p}\right] g_{2}(F(p))+g_{1}(F(p)) \vec{v}_{p}\left[\left.\left(g_{2} \circ F\right)\right|_{p}\right] \\
& =\mathrm{d} F\left(\vec{v}_{p}\right)\left[\left.g_{1}\right|_{F(p)}\right] g_{2}(F(p))+g_{1}(F(p)) \mathrm{d} F\left(\vec{v}_{p}\right)\left[\left.g_{2}\right|_{F(p)}\right] .
\end{aligned}
$$


Observação 1.19. Verifiquemos que $\mathrm{d} F$ leva vetores tangentes reais em vetores tangentes reais. Se $\left.g\right|_{F(p)}$ é um germe de função real em $N,\left.(g \circ F)\right|_{p}$ é um germe de função real em $M$. Assim, se $\vec{v}_{p}$ é um vetor tangente real em $p \in M, \mathrm{~d} F\left(\vec{v}_{p}\right)\left[\left.g\right|_{F(p)}\right]=\vec{v}_{p}\left[\left.(g \circ F)\right|_{p}\right]$ é um número real sempre que $\left.g\right|_{F(p)}$ é um germe de função real.

Observação 1.20. Verifiquemos a linearidade de $\left.\mathrm{d} F\right|_{p}:$ dados $\vec{v}_{1}, \vec{v}_{2} \in \mathrm{T}_{p} M$, $\lambda \in \mathbb{C}$ e $\left.f\right|_{F(p)} \in \mathcal{C}^{\infty}(F(p)), \operatorname{com} f \in \mathcal{C}^{\infty}(M)$,

$$
\begin{aligned}
& \mathrm{d} F\left(\vec{v}_{1}+\lambda \vec{v}_{2}\right)\left[\left.f\right|_{F(p)}\right]=\left(\vec{v}_{1}+\lambda \vec{v}_{2}\right)\left[\left.(f \circ F)\right|_{p}\right] \\
& =\vec{v}_{1}\left[\left.(f \circ F)\right|_{p}\right]+\lambda \vec{v}_{2}\left[\left.(f \circ F)\right|_{p}\right]=\mathrm{d} F\left(\vec{v}_{1}\right)\left[\left.f\right|_{F(p)}\right]+\lambda \mathrm{d} F\left(\vec{v}_{2}\right)\left[\left.f\right|_{F(p)}\right] .
\end{aligned}
$$

Proposição 1.21 (Cálculo do diferencial usando caminhos). Sejam $\alpha:(a, b)$ $\rightarrow M$ uma curva suave na variedade $M$ e $F: M \rightarrow N$ uma aplicação entre variedades. Entãa $\mathrm{d} F\left(\alpha^{\prime}(t)\right)=(F \circ \alpha)^{\prime}(t)$.

Demonstração. Basta calcular $\mathrm{d} F\left(\alpha^{\prime}(t)\right)$ em um germe de função suave arbitrário $\left.g\right|_{F(\alpha(t))}$ :

$$
\begin{aligned}
\mathrm{d} F\left(\alpha^{\prime}(t)\right)\left[\left.g\right|_{F(\alpha(t))}\right] & =\alpha^{\prime}(t)\left[\left.(g \circ F)\right|_{\alpha(t)}\right]=(g \circ F \circ \alpha)^{\prime}(t) \\
& =(F \circ \alpha)^{\prime}(t)\left[\left.g\right|_{F \circ \alpha(t)}\right] . \\
\therefore \mathrm{d} F(\alpha(t)) & =(F \circ \alpha)^{\prime}(t) .
\end{aligned}
$$

Proposição 1.22 (Regra da Cadeia em Variedades). Sejam $F: M \rightarrow N e$ $G: N \rightarrow P$ aplicações suaves entre variedades. Então

$$
\mathrm{d}(G \circ F)=\mathrm{d} G \circ \mathrm{d} F .
$$

Demonstração. Sejam $\vec{v}_{p}$ um vetor tangente qualquer em $\mathrm{T}_{p} M$ e $f \in \mathcal{C}^{\infty}(P)$.

$$
\begin{aligned}
\mathrm{d}(G \circ F)\left(\vec{v}_{p}\right)\left[\left.f\right|_{G \circ F(p)}\right] & =\vec{v}_{p}\left[\left.(f \circ(G \circ F))\right|_{p}\right]=\vec{v}_{p}\left[\left.((f \circ G) \circ F)\right|_{p}\right] \\
& =\mathrm{d} F\left(\vec{v}_{p}\right)\left[\left.(f \circ G)\right|_{F(p)}\right]=\mathrm{d} G\left(\mathrm{~d} F\left(\vec{v}_{p}\right)\right)\left[\left.f\right|_{F \circ G(p)}\right] .
\end{aligned}
$$




\subsubsection{Derivadas parciais de uma aplicação}

Definição 1.23. Seja $F: M \rightarrow N$ uma aplicação suave entre variedades e seja $\vec{x}: U \subset M \rightarrow V \subset \mathbb{R}^{m}, \quad \vec{x}=\left(x_{1}, \ldots, x_{m}\right)$ carta local de $M$. Definimos

$$
\frac{\partial F}{\partial x_{j}}(p)=\mathrm{d} F\left(\left.\frac{\partial}{\partial x_{j}}\right|_{p}\right), \quad \forall p \in U, \quad \forall j \in\{1, \ldots, m\}
$$

Observação 1.24. Se $f \in \mathcal{C}^{\infty}(N)$,

$$
\frac{\partial F}{\partial x_{j}}(p)\left[\left.f\right|_{F(p)}\right]=\mathrm{d} F\left(\left.\frac{\partial}{\partial x_{j}}\right|_{p}\right)\left[\left.f\right|_{F(p)}\right]=\left.\frac{\partial}{\partial x_{j}}\right|_{p}\left[\left.(f \circ F)\right|_{p}\right] .
$$

Exemplo 1.25. Considere $p \in M$ fixo, $F$ como acima. Seja $\vec{y}: U^{\prime} \subset N \rightarrow$ $V^{\prime} \subset \mathbb{R}^{n}$ uma carta local de $N \operatorname{com} F(p) \in U^{\prime}$. Vamos calcular $\partial F / \partial x_{j}(p)$ usando as coordenadas locais $\left(y_{1}, \ldots, y_{n}\right)$ de $N$.

Seja $\Omega=U \cap F^{-1}\left(U^{\prime}\right)$, aberto em $M$. Sejam $\alpha_{j}:(-\delta, \delta) \rightarrow \Omega$, dadas por $\vec{x}\left(\alpha_{j}(t)\right)=\vec{x}(p)+\overrightarrow{e_{j}} t$, onde $\overrightarrow{e_{j}}$ é o $j$-ésimo elemento da base canônica de $\mathbb{R}^{m}$ e $\delta>0$ é pequeno o bastante para que cada $\alpha_{j} \in \Omega$. Então $\alpha_{j}^{\prime}(0)=\left.\partial_{x_{j}}\right|_{p} \mathrm{e}$ podemos calcular (pela proposição 1.21):

$$
\frac{\partial F}{\partial x_{j}}(p)=\mathrm{d} F\left(\left.\frac{\partial}{\partial x_{j}}\right|_{p}\right)=\mathrm{d} F\left(\alpha_{j}^{\prime}(0)\right)=\left(F \circ \alpha_{j}\right)^{\prime}(0) .
$$

Seja $\beta_{j}$ a curva $F \circ \alpha_{j}$. Defina $\beta_{j}^{i}(t)=y_{i}\left(\beta_{j}(t)\right),|t|<\delta$. Defina também $F_{j}: \vec{x}(\Omega) \rightarrow \mathbb{R}$, pela fórmula $F_{j}(T)=y_{j}\left(F\left(\vec{x}^{-1}(T)\right), \quad \forall T \in \vec{x}(\Omega)\right.$. Pela fórmula local da derivada de curva (exemplo 1.16),

$$
\left(F \circ \alpha_{j}\right)^{\prime}(0)=\beta_{j}^{\prime}(0)=\left.\sum_{i=1}^{n}\left(\beta_{j}^{i}\right)^{\prime}(0) \frac{\partial}{\partial y_{i}}\right|_{F(p)}=\left.\sum_{i=1}^{n} \partial_{j}\left(F_{i}\right)(\vec{x}(p)) \frac{\partial}{\partial y_{i}}\right|_{F(p)} .
$$

\subsubsection{Matriz jacobiana}

Definição 1.26. A matriz $A(p)=\left(a_{i j}\right)_{n \times m}$, com $a_{i j}=\partial_{j}\left(F_{i}\right)\left(\vec{x}_{i}(p)\right)$, onde $F_{i}$ é definida como no exemplo anterior (exemplo 1.25), é chamada de matriz jacobiana de $F$ no ponto $p$, em relação às cartas $\vec{x}$ e $\vec{y}$.

Proposição 1.27. Seja $F: M \rightarrow N$ uma aplicação suave entre variedades e sejam $\vec{x}: U \subset M \rightarrow V \subset \mathbb{R}^{m}$ e $\vec{y}: U^{\prime} \subset N \rightarrow V^{\prime} \subset \mathbb{R}^{n}$ cartas locais de $M$ e $N$, respectivamente, com $p \in M$ um ponto tal que $p \in U$ e $F(p) \in U^{\prime}$. 
Considere $A(p)=\left(a_{i j}\right)_{n \times m}$ a matriz jacobiana de $F$ em $p$, em relação às cartas $\vec{x}$ e $\vec{y}$. Então o diferencial de $F$ no ponto $p$,

$$
\left.\mathrm{d} F\right|_{p}: \mathrm{T}_{p} M \longrightarrow \mathrm{T}_{F(p)} N,
$$

é determinado por $A(p)$, pela fórmula

$$
\left.\mathrm{d} F\right|_{p}\left(\vec{v}_{p}\right)=\left.\sum_{j=1}^{m} \sum_{i=1}^{n} \vec{v}_{p}\left[\left.x_{j}\right|_{p}\right] a_{i j} \frac{\partial}{\partial y_{j}}\right|_{p} .
$$

Demonstração. De fato, usando a proposição 1.13 e o exemplo 1.25:

$$
\begin{aligned}
& \mathrm{d} F\left(\vec{v}_{p}\right)=\mathrm{d} F\left(\left.\sum_{j=1}^{m} \vec{v}_{p}\left[\left.x_{j}\right|_{p}\right] \frac{\partial}{\partial x_{j}}\right|_{p}\right)=\sum_{j=1}^{m} \vec{v}_{p}\left[\left.x_{j}\right|_{p}\right] \frac{\partial F}{\partial x_{j}}(p) \\
&=\left.\sum_{j=1}^{m} \vec{v}_{p}\left[\left.x_{j}\right|_{p}\right] \sum_{i=1}^{n} a_{i j} \frac{\partial}{\partial y_{i}}\right|_{p}
\end{aligned}
$$

Corolário 1.28. O posto de $F$ em $p$ é o posto da matriz jacobiana $A(p)$ de $F$, em relação a cartas locais quaisquer.

Observação 1.29. A matriz jacobiana de $F$ em $p$, em relação a $\vec{x}$ e $\vec{y}$, é precisamente a matriz jacobiana em $\vec{x}(p)$ de $F_{\vec{x} \vec{y}}$, que é a aplicação entre espaços euclidianos definida por $F_{\vec{x} \vec{y}}(T)=\vec{y}\left(F\left(\vec{x}^{-1}(T)\right)\right)$.

\subsection{Teoremas da imersão, da submersão e do posto}

Sejam $M$ e $N$ variedades $\mathcal{C}^{\infty}$ de dimensão $m$ e $n$, respectivamente, e seja $F: M \rightarrow N$ uma aplicação $\mathcal{C}^{\infty}$. Seja $p \in M$.

Seguem três resultados básicos do cálculo em variedades:

Teorema 1.30 (Imersão). Se F tem posto $m$ em $p$, então existem cartas

$$
\begin{aligned}
& \vec{x}: \quad U \subset M \longrightarrow V \subset \mathbb{R}^{m}, \quad p \in U e \\
& \vec{y}: \quad U^{\prime} \subset N \longrightarrow V^{\prime} \subset \mathbb{R}^{n}, \quad f(p) \in U^{\prime}
\end{aligned}
$$

de $M$ e $N$ tais que $F(U) \subset U^{\prime}, V^{\prime}=V \times(-\delta, \delta)^{n-m}$ com $\delta>0$ e, sendo $\tilde{F}=\left.F\right|_{U \rightarrow U^{\prime}}$,

$$
\vec{y} \circ \tilde{F}=\left.(x_{1}, \ldots, x_{m}, \underbrace{0, \ldots, 0}_{n-m})\right|_{U \rightarrow V^{\prime}} .
$$


Teorema 1.31 (Submersão). Se F tem posto $n$ em $p$, então existem cartas

$$
\begin{aligned}
& \vec{x}: \quad U \subset M \longrightarrow V \subset \mathbb{R}^{m}, \quad p \in U e \\
& \vec{y}: \quad U^{\prime} \subset N \longrightarrow V^{\prime} \subset \mathbb{R}^{n}, \quad f(p) \in U^{\prime}
\end{aligned}
$$

de $M$ e $N$ tais que $F(U) \subset U^{\prime}, V=V^{\prime} \times(-\delta, \delta)^{m-n}$ com $\delta>0$ e, sendo $\tilde{F}=\left.F\right|_{U \rightarrow U^{\prime}}$,

$$
\vec{y} \circ \tilde{F}=\left.\left(x_{1}, \ldots, x_{n}\right)\right|_{U \rightarrow V^{\prime}} .
$$

Teorema 1.32 (Posto). Se F tem posto $k$ em uma vizinhança de p, então existem cartas

$$
\begin{aligned}
& \vec{x}: \quad U \subset M \longrightarrow V \subset \mathbb{R}^{m}, \quad p \in U e \\
& \vec{y}: \quad U^{\prime} \subset N \longrightarrow V^{\prime} \subset \mathbb{R}^{n}, \quad f(p) \in U^{\prime}
\end{aligned}
$$

de $M$ e $N$ e um aberto $W \subset \mathbb{R}^{k}$ tais que $F(U) \subset U^{\prime}, V=W \times(-\delta, \delta)^{m-k}$, $V^{\prime}=W \times(-\delta, \delta)^{n-k} \operatorname{com} \delta>0$ e, sendo $\tilde{F}=\left.F\right|_{U \rightarrow U^{\prime}}$,

$$
\vec{y} \circ \tilde{F}=\left.(x_{1}, \ldots, x_{k}, \underbrace{0, \ldots, 0}_{n-k})\right|_{U \rightarrow V^{\prime}} .
$$

Para demonstrar estes teoremas, basta usar nas coordenadas locais os teoremas respectivos (forma local das imersões, forma local das submersões e teorema do posto) do cálculo em $\mathbb{R}^{n}$. Uma demonstração destes se encontra em [3].

\subsection{Subvariedades imersas}

Definição 1.33. Uma subvariedade imersa (que às vezes chamaremos simplesmente de subvariedade) da variedade $M$ é uma variedade $N$ cujo conjunto dos pontos é subconjunto do conjunto dos pontos de $M$ e que satisfaz a seguinte propriedade: a inclusão em $M$,

$$
\begin{aligned}
i: N & \longrightarrow M \\
p & \longmapsto p
\end{aligned},
$$

é uma imersão, isto é, $i$ é uma aplicação suave (e, portanto, contínua) cujo diferencial em cada $p \in N$, di $\left.\right|_{p}: \mathrm{T}_{p} N \rightarrow \mathrm{T}_{p} M$, é injetivo.

Observação 1.34. Como $i$ é injetiva e imersão, di será injetivo. O fato de $\mathrm{d} i$ ser injetivo implica que di é uma bijeção sobre sua imagem, isto é, di faz uma relação biunívoca entre o fibrado tangente $\mathrm{T} N$ e $\mathrm{d} i(\mathrm{~T} N) \subset \mathrm{T} M$. Para cada 
$p \in N$, a imagem di $\left(\mathrm{T}_{p} N\right)$ é um subespaço vetorial de $\mathrm{T}_{p} M$ de dimensão igual à dimensão de $N$.

Assim, vamos identificar cada vetor tangente $\vec{v}_{p} \in \mathrm{T}_{p} N$ com $\mathrm{d} i\left(\vec{v}_{p}\right) \in$ $\mathrm{T}_{p} M$. Com esta identificação, podemos tratar os vetores tangentes da subvariedade imersa como se fossem vetores tangentes da variedade.

Observação 1.35. Para verificar que uma aplicação $F$ é uma imersão, basta verificar que a matriz jacobiana tem sempre posto igual a $\operatorname{dim}(\operatorname{Dom}(F))$.

Observação 1.36. O fato de a inclusão $i: N \rightarrow M$ ser contínua implica que a topologia de $N$ (como variedade) é mais fina do que a topologia de $N$ como subespaço topológico de $M$.

Observação 1.37. Aplicando o teorema da imersão na inclusão, obtemos uma caracterização da estrutura diferenciável das subvariedades imersas: dados $p \in N$, sendo $N$ subvariedade imersa de $M$, existem cartas locais:

$$
\begin{aligned}
& \vec{x}: \quad U \subset N \longrightarrow V \subset \mathbb{R}^{n} \mathrm{e} \\
& \vec{y}: \quad U^{\prime} \subset M \longrightarrow V^{\prime} \subset \mathbb{R}^{m}
\end{aligned}
$$

de $N$ e $M$ respectivamente tais que $p \in U \subset U^{\prime}, V^{\prime}=V \times(-\delta, \delta)^{m-n}$ para $\operatorname{algum} \delta>0 \mathrm{e}$

$$
\left.\vec{y}\right|_{U \rightarrow \vec{y}(U)}=(x_{1}, \ldots, x_{n}, \underbrace{0, \ldots, 0}_{m-n}) .
$$

Note que nem sempre podemos exigir que $U^{\prime} \cap N=U$.

Proposição 1.38 (Imagem de Imersão Quociente). Seja $F: M \rightarrow N$ uma imersão entre variedades. Se F é uma aplicação quociente, isto é, se $F^{-1}(F(U))$ é aberto em $M$ para todo aberto $U$ em $M$, então:

(i) $F(M)$ é uma subvariedade imersa de $N$ com a topologia co-induzida por $F$ (isto é, com a topologia dos conjuntos cuja imagem inversa por $F$ é aberta).

(ii) O espaço tangente de $F(M)$ em um ponto $F(q)$ é dado por $\mathrm{T}_{F(q)} F(M)=$ $\mathrm{d} F\left(\mathrm{~T}_{q} M\right)$.

Demonstração. Vamos construir uma estrutura diferenciável para o espaço topológico $(F(M), \tau)$, onde

$$
\tau=\left\{U \subset N \mid F^{-1}(U) \text { é aberto em } M\right\} .
$$

Usaremos o teorema da imersão em $F$ : dado $p \in M$, existem cartas locais:

$$
\begin{array}{ll}
\vec{x}_{p}: U_{p} \stackrel{a b .}{\subset} M \longrightarrow V_{p} \stackrel{a b .}{\subset} \mathbb{R}^{m}, \quad p \in U_{p}, \quad \vec{x}_{p}=\left(x_{1}^{p}, \ldots, x_{m}^{p}\right) \quad \mathrm{e} \\
\vec{y}_{p}: U_{p}^{\prime} \stackrel{a b .}{\subset} N \longrightarrow V_{p}^{\prime} \stackrel{a b .}{\subset} \mathbb{R}^{n}, \quad F(p) \in U_{p}^{\prime}, \quad \vec{y}_{p}=\left(y_{1}^{p}, \ldots, y_{n}^{p}\right),
\end{array}
$$


com $V_{p}^{\prime}=V_{p} \times\left(-\delta_{p}, \delta_{p}\right)^{n-m}$, para algum $\delta_{p}>0$, onde $n=\operatorname{dim} N$ e $m=$ $\operatorname{dim} M, F\left(U_{p}\right) \subset U_{p}^{\prime} \mathrm{e}$

$$
\vec{y}_{p}\left(F\left(p^{\prime}\right)\right)=(x_{1}^{p}, \ldots, x_{m}^{p}, \underbrace{0, \ldots, 0}_{n-m})\left(p^{\prime}\right), \quad \forall p^{\prime} \in U_{p} .
$$

Para servir de carta local de $F(M)$ em torno do ponto $F(p)$, definiremos a aplicação

$$
\begin{aligned}
\phi_{p}: F\left(U_{p}\right) & \longrightarrow V_{p} \\
q & \longmapsto\left(y_{1}^{p}(q), \ldots, y_{m}^{p}(q)\right),
\end{aligned}
$$

cujo domínio $F\left(U_{p}\right)$ é aberto em $(F(M), \tau)$ devido a $F$ ser quociente. Note que $\phi_{p}\left(F\left(p^{\prime}\right)\right)=\left(x_{1}^{p}\left(p^{\prime}\right), \ldots, x_{m}^{p}\left(p^{\prime}\right)\right), \quad \forall p^{\prime} \in U_{p}$. Mostremos que $\phi_{p}$ é um homeomorfismo entre $F\left(U_{p}\right)$ e $V_{p}$.

$\phi_{p}$ é contínua: dado $O \stackrel{a b .}{\subset} V_{p} \stackrel{a b .}{\subset} \mathbb{R}^{m}$,

$$
\begin{aligned}
\phi_{p}^{-1}(O) & =\left\{q \in F\left(U_{p}\right) \mid\left(y_{1}^{p}, \ldots, y_{m}^{p}\right)(q) \in O\right\} \\
& =\left\{F\left(p^{\prime}\right) \in V_{p} \mid\left(x_{1}^{p}, \ldots, x_{m}^{p}\right)\left(p^{\prime}\right) \in O\right\}=F\left(\vec{x}_{p}^{-1}(O)\right) .
\end{aligned}
$$

Como $\vec{x}_{p}: U_{p} \rightarrow V_{p}$ é contínua, a imagem inversa $\vec{x}_{p}^{-1}(O)$ é aberta em $U_{p}$. Como $F$ é uma aplicação quociente, $F\left(\vec{x}_{p}^{-1}(O)\right)$ é aberto em $F(M)$ e está contido em $F\left(U_{p}\right)$, que, por sua vez, é aberto em $F(M)$. Concluímos que $\phi_{p}^{-1}(O)=F\left(\vec{x}_{p}^{-1}(O)\right)$ é aberto em $F\left(U_{p}\right)$.

$\phi_{p}$ é aberta: Seja $O^{\prime} \stackrel{a b}{\complement} F\left(U_{p}\right)$. Podemos escrever $O=F(\Omega)$, com $\Omega=$ $F^{-1}\left(O^{\prime}\right) \cap U_{p} \stackrel{a b .}{\complement} U_{p}$. Então, $\phi_{p}\left(O^{\prime}\right)=\phi_{p}(F(\Omega))=\vec{x}_{p}(\Omega)$, que é aberto em $V_{p}$.

Como $\phi_{p}$ é contínua, aberta e bijetiva, $\phi_{p}$ é um homeomorfismo.

Agora, verifiquemos que as cartas locais construídas desta forma são compatíveis. Sejam $\phi_{p_{1}}: F\left(U_{p_{1}}\right) \rightarrow V_{p_{1}}$ e $\phi_{p_{2}}: F\left(U_{p_{2}}\right) \rightarrow V_{p_{2}}$, construídas como acima com cartas locais $x^{p_{1}}, x^{p_{2}}, y^{p_{1}}, y^{p_{2}}$ de $M$ e $N$ associadas. Mostremos que a mudança de carta

$$
\begin{array}{cl}
\Psi: \phi_{p_{1}}\left(F\left(U_{p_{1}}\right) \cap F\left(U_{p_{2}}\right)\right) & \longrightarrow \phi_{p_{2}}\left(F\left(U_{p_{1}}\right) \cap F\left(U_{p_{2}}\right)\right) \\
p & \longmapsto \phi_{p_{2}}\left(\phi_{p_{1}}(p)\right)
\end{array}
$$

é uma aplicação suave. De fato, pois:

$$
\Psi\left(t_{1}, \ldots, t_{m}\right)=\left(\eta_{1}, \ldots, \eta_{m}\right)(t_{1}, \ldots, t_{m}, \underbrace{0, \ldots, 0}_{n-m}),
$$


onde $\eta: \vec{y}_{p_{1}}\left(U_{p_{1}}^{\prime} \cap U_{p_{2}}^{\prime}\right) \rightarrow \vec{y}_{p_{2}}\left(U_{p_{1}}^{\prime} \cap U_{p_{2}}^{\prime}\right)$ é a mudança de coordenada de $\vec{y}_{p_{1}}$ para $\vec{y}_{p_{2}}$, uma aplicação suave.

Assim, as aplicações da forma $\phi_{p}$ induzem uma estrutura diferenciável sobre o espaço topológico $(F(M), \tau)$ formando uma variedade.

Agora, devemos mostrar que a variedade $F(M)$ construída acima é uma subvariedade imersa de $N$.

Seja $i: F(M) \rightarrow N$ a inclusão. Dado $q=F(p) \in F(M)$, consideremos as cartas locais $\phi_{p}: F\left(U_{p}\right) \rightarrow V_{p}$ e $\vec{y}^{p}: U_{p}^{\prime} \rightarrow V_{p}^{\prime}$, definidas como na construção acima. A expressão de $i$ em coordenadas locais é

$$
\vec{y}^{p}\left(i\left(\phi_{p}^{-1}\left(t_{1}, \ldots, t_{m}\right)\right)\right)=\left(t_{1}, \ldots, t_{m}, 0, \ldots, 0\right),
$$

o que nos dá uma matriz jacobiana da forma $\left(\begin{array}{c|c}I_{m \times m} & 0 \\ \hline 0 & 0\end{array}\right)$, onde $I_{m \times m}$ é a matriz identidade de tamanho $m$ e os zeros representam blocos nulos. Isto mostra que $i$ é uma imersão.

$\mathrm{d} F\left(\left.\partial_{x_{j}}\right|_{p}\right)=\left.\partial_{y_{j}}\right|_{p}$, pela equação (1.39). Assim, $\mathrm{T}_{F(p)} F(M)=\mathrm{d} F\left(\mathrm{~T}_{p} M\right)$.

Corolário 1.40. A imagem de uma imersão injetiva é uma subvariedade imersa na topologia co-induzida.

\subsubsection{Unicidade}

\section{Subvariedades diferentes com o mesmo conjunto de pontos}

Definição 1.41 (Subvariedade aberta). Seja $M$ uma variedade suave e $U \subset$ $M$ um aberto em $M$. Considere $U$ dotado da topologia que é herdada de $M$.

A restrição da estrutura diferenciável de $M$ para $U$ é uma estrutura diferenciável em $U$. A variedade $U$ assim obtida é uma subvariedade imersa de $U$. Uma subvariedade deste tipo será chamada de subvariedade aberta de $M$.

Observação 1.42. Para verificar que a subvariedade aberta é mesmo uma subvariedade imersa, basta considerar que a matriz jacobiana da inclusão, escolhendo-se em $U$ uma carta que é a restrição da carta escolhida em $M$, é a matriz identidade.

Proposição 1.43 (União disjunta de subvariedades imersas). Sejam $\left\{N_{j}\right\}_{j \in J}$ subvariedades imersas disjuntas de $M$, todas com a mesma dimensão $n \leq$ $\operatorname{dim} M$. Seja $N=\bigcup_{j \in J} N_{j}$ a união, dotada da topologia gerada pela união das topologias das $N_{j}$. Então a união das estruturas diferenciáveis das $N_{j}$ gera uma estrutura diferenciável para $N$, de dimensão $n$.

A variedade $N$ assim construída é subvariedade imersa de $M$. 
Demonstração. As estruturas diferenciáveis das $N_{j}$ são compatíveis entre si, pois neste caso as cartas de subvariedades distintas não se interceptam. Como as cartas das $N_{j}$, tomadas em conjunto, cobrem $N$, é possível gerar uma estrutura diferenciável em $N$ a partir delas.

Afirmação: a inclusão $i: N \rightarrow M$ é uma imersão. De fato, pois a matriz jacobiana de $i$ em cada ponto coincide com a matriz jacobiana de alguma inclusão $i_{j}: N_{j} \rightarrow M$ (escolhendo-se as cartas convenientemente) e esta terá posto $n$.

Exemplo 1.44. (i) Considere o aberto $Q=(0,1) \times(0,1) \subset \mathbb{R}^{2}$, visto apenas como conjunto de pontos e não como variedade. Seja $N_{1}$ a subvariedade aberta de $\mathbb{R}^{2}$ que tem $Q$ como conjunto de pontos.

Para cada $y \in(0,1)$, seja $R_{y}$ a imagem da imersão injetiva $\alpha_{y}:(0,1) \rightarrow$ $\mathbb{R}^{2}$ definida por $\alpha_{y}(t)=(t, y)$. Isto é simplesmente o segmento de reta $(0,1) \times\{y\}$, uma subvariedade imersa unidimensional de $\mathbb{R}^{2}$.

Seja $N_{2}$ a união disjunta $\bigcup_{y \in(0,1)} R_{y}$ de subvariedades. Assim como $N_{1}$, $N_{2}$ é uma subvariedade imersa de $\mathbb{R}^{2}$ com o conjunto de pontos $Q$, mas a dimensão de $N_{2}$ é 1. A topologia de $N_{2}$ é estritamente mais fina que a topologia de $N_{1}$.

(ii) Ainda no plano cartesiano, seja $N_{3}$ a união disjunta dos seguintes segmentos de reta: $(-1,0) \times\{0\},(0,1) \times\{0\}$ e $\{0\} \times(-1,1)$, e seja $N_{4}$ a união disjunta dos segmentos: $(-1,1) \times\{0\},\{0\} \times(-1,0)$ e $\{0\} \times(0,1)$.

$N_{3}$ e $N_{4}$ são subvariedades com o mesmo conjunto de pontos e a mesma dimensão, mas têm topologias diferentes e, portanto, estruturas diferenciáveis diferentes. As topologias de $N_{3}$ e $N_{4}$ não são comparáveis.

\section{Subvariedade de dimensão maximal}

Proposição 1.45. Uma subvariedade imersa de dimensão maximal é sempre uma subvariedade aberta.

Demonstração. Seja $N \subset M$ uma subvariedade imersa com $\operatorname{dim} N=\operatorname{dim} M$ $=m$. Como $i: N \rightarrow M$ é uma imersão, o diferencial d $\left.i\right|_{p}$ tem posto $m$ para cada $p \in N$. Ou seja, $\left.d i\right|_{p}$ é isomorfismo para cada $p \in N$. Aplicando o teorema da função inversa em $i$, encontramos vizinhanças $U_{p} \stackrel{a b .}{\subset} N, V_{p} \stackrel{a b .}{\complement}$ $M$ de $p$ tais que $\left.i\right|_{U_{p} \rightarrow V_{p}}$ é difeomorfismo - em particular, como $V_{p}$ é uma vizinhança aberta de $p$ em $M$, isto implica que $i(N)$ é aberto em $M$, ou seja, o conjunto de pontos de $N$ é aberto em $M$. 
Seja $N_{2}$ a subvariedade aberta de $M$ no conjunto de pontos de $N$. Como as cartas de $N_{2}$ também são cartas de $M$, a aplicação

$$
\begin{aligned}
I: N & \longrightarrow N_{2} \\
p & \longmapsto p
\end{aligned}
$$

terá a mesma matriz jacobiana que $i$ nos pontos de $p$, com a escolha conveniente das cartas. Assim, $\left.\mathrm{d} I\right|_{p}$ será um isomorfismo para cada $p$ e, pelo teorema da função inversa, $I$ será um difeomorfismo, o que implica que $N=N_{2}$.

Corolário 1.46. Duas subvariedades com o mesmo conjunto de pontos são iguais, desde que elas tenham dimensão maximal.

\section{Subvariedades com topologias comparáveis}

Proposição 1.47. Sejam $N_{1}$ e $N_{2}$ duas subvariedades imersas de $M$ como mesmo conjunto de pontos e suponha que a topologia de $N_{1}$ é mais fina que a topologia de $N_{2}$. Então $N_{1}$ é subvariedade de $N_{2}$.

Demonstração. Sejam $N_{1}$ e $N_{2}$ como na hipótese. Primeiro, vamos mostrar que a aplicação

$$
\begin{aligned}
I_{12}: N_{1} & \longrightarrow N_{2} \\
p & \longmapsto p
\end{aligned}
$$

é suave. A continuidade de $I_{12}$ é consequência de a topologia de $N_{1}$ ser mais fina que a topologia de $N_{2}$. Seja $p \in N_{1}=N_{2}$. Sejam

$$
\begin{array}{ll}
\vec{x}: U_{1} \subset N_{1} \longrightarrow V_{1} \subset \mathbb{R}^{n_{1}}, & n_{1}=\operatorname{dim} N_{1}, \\
\vec{y}: U_{1}^{\prime} \subset M \longrightarrow V_{1}^{\prime} \subset \mathbb{R}^{m}, & m=\operatorname{dim} M, \\
U_{1} \subset U_{1}^{\prime}, & V_{1}^{\prime}=V_{1} \times\left(-\delta_{1}, \delta_{1}\right)^{m-n_{1}}, \quad \delta>0
\end{array}
$$

e

$$
\begin{array}{ll}
\vec{z}: U_{2} \subset N_{2} \longrightarrow V_{2} \subset \mathbb{R}^{n_{2}}, & n_{2}=\operatorname{dim} N_{2}, \\
\vec{w}: U_{2}^{\prime} \subset M \longrightarrow V_{2}^{\prime} \subset \mathbb{R}^{m}, & \\
U_{2} \subset U_{2}^{\prime}, & V_{2}^{\prime}=V_{2} \times\left(-\delta_{2}, \delta_{2}\right)^{m-n_{1}}, \quad \delta>0
\end{array}
$$

cartas locais em $p$ tais que

$$
\begin{aligned}
\left.\vec{y}\right|_{U_{1} \rightarrow \vec{y}\left(U_{1}\right)} & =(x_{1}, \ldots, x_{n_{1}}, \underbrace{0, \ldots, 0}_{m-n_{1}}) \mathrm{e} \\
\left.\vec{w}\right|_{U_{2} \rightarrow \vec{w}\left(U_{2}\right)} & =(z_{1}, \ldots, z_{n_{2}}, \underbrace{0, \ldots, 0}_{m-n_{2}}) .
\end{aligned}
$$


A intersecção $\Omega^{\prime}=U_{1}^{\prime} \cap U_{2}^{\prime}$ contém $\Omega=U_{1} \cap U_{2}$. Como a topologia de $N_{1}$ é mais fina que a de $N_{2}, \Omega$ é aberto em $N_{1}$. Seja $\Psi: \vec{y}\left(\Omega^{\prime}\right) \rightarrow \vec{w}\left(\Omega^{\prime}\right)$ a mudança de carta de $\vec{y}$ para $\vec{w}$. A função $I_{12}$ se expressa, em função das cartas $\vec{x}$ e $\vec{z}$, como:

$$
\vec{z}\left(I_{12}\left(\vec{x}^{-1}\left(t_{1}, \ldots, t_{n_{1}}\right)\right)\right)=\left(\Psi_{1}, \ldots, \Psi_{n_{2}}\right)(t_{1}, \ldots, t_{n_{1}}, \underbrace{0, \ldots, 0}_{m-n_{1}}),
$$

definida no aberto $\vec{x}(\Omega)$. Isto faz de $I_{12}$ uma função suave em $p$. Como $p$ é arbitrário, $I_{12}$ é suave.

Em segundo lugar, vamos calcular o posto de $I_{12}$. Seja $A$ a matriz jacobiana $m \times m$ de $\Psi$ em $p \in \Omega$.

$$
A=\left(\begin{array}{ccc}
\left.\frac{\partial w_{1}}{\partial y_{1}}\right|_{p} & \cdots & \left.\frac{\partial w_{1}}{\partial y_{m}}\right|_{p} \\
\vdots & \ddots & \vdots \\
\left.\frac{\partial w_{m}}{\partial y_{1}}\right|_{p} & \cdots & \left.\frac{\partial w_{m}}{\partial y_{m}}\right|_{p}
\end{array}\right) \quad \text { (jacobiana da mudança de coordenadas) }
$$

Como as coordenadas $w_{j}, j>n_{2}$ são nulas em $\Omega$, e os vetores tangentes $\left.\partial_{y_{k}}\right|_{p}$, com $1 \leq k \leq n_{1}$ podem ser escritos como derivada de uma curva contida em $\Omega$, temos:

$$
\left.\frac{\partial w_{j}}{\partial y_{k}}\right|_{p}=0, \quad \forall j, k \text { tais que } n_{2}<j \leq m \text { e } 1 \leq k \leq n_{1} .
$$

Assim, $A$ terá a seguinte forma em blocos: $\left(\begin{array}{c|c}\left(A_{1}\right)_{n_{2} \times n_{1}} & A_{2} \\ \hline 0 & A_{3}\end{array}\right)$. Note que a submatriz $A_{1}$ é a matriz jacobiana de $I_{12}$ em $p$, nas coordenadas locais $\vec{x} \mathrm{e}$ $\vec{z}$.

O posto de $A$ é $m$, pois $A$ é a matriz jacobiana de um difeomorfismo. Usando-se o fato de que o posto de uma matriz é igual ao número de colunas linearmente independentes, concluímos que o posto de $A_{1}$ é igual a $n_{1}$. Como $p$ é arbitrário, o posto de $I_{12}$ é sempre igual a $n_{1}$ e, portanto, $I_{12}$ é uma imersão, o que prova a proposição.

Corolário 1.48. Se duas subvariedades $N_{1}, N_{2}$ de $M$ têm o mesmo conjunto de pontos e a mesma topologia, então elas são iguais.

Demonstração. Temos que $N_{1}$ é subvariedade de $N_{2}$ e $N_{2}$ é subvariedade de $N_{1}$. Isto significa que $\operatorname{dim} N_{2}=\operatorname{dim} N_{1}$ e, ainda, que a identidade entre $N_{1}$ e $N_{2}$ é um difeomorfismo. Logo, $N_{1}=N_{2}$. 
Corolário 1.49 (Mesma dimensão e topologias comparáveis). Se duas subvariedades $N_{1}$ e $N_{2}$ de $M$ têm o mesmo conjunto de pontos, a mesma dimensão e suas topologias são comparáveis, então $N_{1}=N_{2}$

Demonstração. Suponha que a topologia de $N_{1}$ é mais fina que a topologia de $N_{2}$. Então $N_{1}$ é uma subvariedade de dimensão maximal de $N_{2}$. Pela proposição $1.45, N_{1}$ é a subvariedade aberta de $N_{2}$ com o mesmo conjunto de pontos de $N_{2}$, ou seja: é a própria $N_{2}$.

Se a topologia de $N_{2}$ é mais fina, procede-se analogamente

\section{Subvariedades mergulhadas}

Definição 1.50. Uma subvariedade imersa $N \subset M$ cuja topologia coincide com a topologia do subespaço herdada de $M$ é chamada de subvariedade mergulhada.

Exemplo 1.51. Uma subvariedade aberta é subvariedade mergulhada.

Observação 1.52. Pela proposição 1.48, a estrutura diferenciável de uma subvariedade mergulhada é unicamente definida. Em outras palavras, duas subvariedades mergulhadas com o mesmo conjunto de pontos sempre serão iguais.

\subsubsection{União de subvariedades imersas}

Proposição 1.53. Seja $C=\left\{N_{j}\right\}_{j \in J}$ uma familia indexada de subvariedades imersas de M. Suponha que:

(i) As subvariedades têm todas a mesma dimensão, $n$.

(ii) A intersecção de duas subvariedades quaisquer $N_{j_{1}}$ e $N_{j_{2}}$ é aberta, tanto em relação à topologia de $N_{j_{1}}$ quanto em relação à topologia de $N_{j_{2}}$.

(iii) As topologias das subvariedades são compativeis, isto é, dados $j_{1}, j_{2} \in$ $J$, e denotando $\Omega:=N_{j_{1}} \cap N_{j_{2}}$, as restrições das topologias de $N_{j_{1}}$ e $N_{j_{2}}$ a $\Omega$ são iguais.

Então $N:=\bigcup_{j \in J} N_{j}$ é uma subvariedade imersa de $M$, com a topologia gerada pela união das topologias das $N_{j}$ e a estrutura diferenciável gerada pela união das estruturas diferenciáveis das $N_{j}$. Se $p \in N_{j_{0}}$ para algum $j_{0} \in J$, o espaço tangente de $N$ em $p$ é dado por $\mathrm{T}_{p} N=\mathrm{T}_{p} N_{j_{0}}$. 
Demonstração. Para mostrar que a união é uma variedade, basta mostrar que as estruturas diferenciáveis das partes $N_{j} \in C$ são compatíveis. Isto é consequência da proposição 1.48 - que subvariedades imersas com a mesma topologia são iguais.

Para mostrar que a união é uma subvariedade imersa, usamos o mesmo argumento que foi usado na proposição 1.43.

Observação 1.54. Toda subvariedade imersa pode ser decomposta em abertos que são subvariedades mergulhadas: de fato, seja $N$ uma $n$-subvariedade imersa da $m$-variedade $M$. Dado $p \in N$, o teorema da imersão nos dá cartas

$$
\begin{gathered}
\vec{x}: U \subset N \rightarrow V \subset \mathbb{R}^{n} \quad \text { de } N \text { e } \\
\vec{y}: U^{\prime} \subset M \rightarrow V^{\prime} \subset \mathbb{R}^{m} \quad \text { de } M,
\end{gathered}
$$

com $p \in U$, tais que $U \subset U^{\prime}, V^{\prime}=V \times(-\delta, \delta)^{m-n} \mathrm{e}$

$$
\left.\vec{y}\right|_{U \rightarrow \vec{y}(U)}=(x_{1}, \ldots, x_{n}, \underbrace{0, \ldots, 0}_{m-n}) .
$$

Se considerarmos $U$ como subvariedade aberta de $N$, veremos que $U$ é uma subvariedade mergulhada de $M$ : pois, dado um aberto $\mathcal{O} \subset U$,

$$
\mathcal{O}=\vec{y}^{-1}\left(\vec{x}(\mathcal{O}) \times\{0\}^{m-n}\right)=U \cap \underbrace{\vec{y}^{-1}\left(\vec{x}(\mathcal{O}) \times(-\delta, \delta)^{m-n}\right)}_{\text {aberto em M }} .
$$

Ou seja, a topologia da variedade $U$ é menos fina do que a topologia do subespaço que $U$ herda de $M$. Por se tratar de uma subvariedade imersa, isto implica que as topologias são iguais.

Variando $p$, obtemos subvariedades abertas $U_{p}$ de $N$, todas de dimensão $n$ e com topologias compatíveis (pois são subvariedades abertas de $N$ ) que são subvariedades mergulhadas de $M$ e cobrem $N$.

\subsubsection{Restrição de campo vetorial a subvariedade}

Definição 1.55. Seja $X \in \mathfrak{X}(M)$ um campo vetorial real na variedade $M \mathrm{e}$ $N \subset M$ uma subvariedade imersa de $M$. Dizemos que $X$ é tangente a $N$ se $\left.X\right|_{p} \in \mathrm{T}_{p} N, \quad \forall p \in N$.

Proposição 1.56 (Restrição de campo escalar à subvariedade). Sejam $f \in$ $\mathcal{C}^{\infty}(M)$ e $N$ subvariedade de $M$. Então $\left.f\right|_{N} \in \mathcal{C}^{\infty}(N)$.

Demonstração. A continuidade de $\left.f\right|_{N}$ segue do fato de a topologia de $N$ ser mais fina do que a topologia do subespaço herdada de $M$. Dado $p \in N$, mostremos que $\left.f\right|_{N}$ é suave em $p$. 
Sejam $\vec{x}: U \subset N \rightarrow V \subset \mathbb{R}^{n}$ e $\vec{y}: U^{\prime} \subset M \rightarrow V^{\prime} \subset \mathbb{R}^{m}$ cartas locais de $N$ e $M$, respectivamente, tais que

$$
\begin{array}{rlrl}
p & \in U, & V^{\prime} & =V \times(-\delta, \delta)^{m-n}, \\
U \subset U^{\prime}, & \vec{y}(U) & =V \times\{0\}^{m-n}
\end{array}
$$

e

$$
\vec{y}(q)=(x_{1}, \ldots, x_{n}, \underbrace{0, \ldots, 0}_{m-n})(q), \quad \forall q \in N .
$$

Então

$$
\left.\left(\left.f\right|_{N}\right)\right|_{U} \circ \vec{x}^{-1}\left(t_{1}, \ldots, t_{n}\right)=f\left(\vec{y}^{-1}(t_{1}, \ldots, t_{m}, \underbrace{0, \ldots, 0}_{m-n})\right), \quad \forall\left(t_{1}, \ldots, t_{n}\right) \in V .
$$

Assim, como $\left.f\right|_{U^{\prime}} \circ \vec{y}^{-1}$ é suave, $\left.f\right|_{N}$ é suave em $p$.

Proposição 1.57 (Restrição de campo vetorial à subvariedade). Seja X um campo vetorial real definido na variedade suave $M$ e tangente à subvariedade $N \subset M$. Existe um único campo $\left.X\right|_{N} \in \mathfrak{X}(N)$ tal que, para toda $f \in \mathcal{C}^{\infty}(M)$,

$$
\left.X\right|_{N}\left(\left.f\right|_{N}\right)=\left.(X f)\right|_{N},
$$

e ele é definido por $\left.\left(\left.X\right|_{N}\right)\right|_{p}=\left.X\right|_{p}, \quad \forall p \in N$.

Demonstração. Quando a subvariedade $N$ é uma subvariedade aberta, basta usar a proposição 1.9. Façamos o caso geral.

Existência: Seja $i: N \rightarrow M$ a inclusão. Definiremos $\left.X\right|_{N}$ como o campo vetorial que satisfaz $\left.\left(\left.X\right|_{N}\right)\right|_{p}=(\mathrm{d} i)^{-1}\left(\left.X\right|_{p}\right)$. Para que este campo exista basta mostrar que, $\forall g \in \mathcal{C}^{\infty}(N)$, a função $q \mapsto(\mathrm{d} i)^{-1}\left(\left.X\right|_{q}\right)\left[\left.g\right|_{q}\right]$ é suave. Fixado $p \in N$, mostremos que esta função é suave em $p$.

Sejam $\vec{x}: U \subset N \rightarrow V \subset \mathbb{R}^{n}$ e $\vec{y}: U^{\prime} \subset M \rightarrow V^{\prime} \subset \mathbb{R}^{m}$ cartas locais de $N$ e $M$, respectivamente, tais que

$$
\begin{array}{rlrl}
p & \in U, & V^{\prime} & =V \times(-\delta, \delta)^{m-n}, \\
U \subset U^{\prime}, & \vec{y}(U) & =V \times\{0\}^{m-n}
\end{array}
$$

e

$$
\vec{y}(q)=(x_{1}, \ldots, x_{n}, \underbrace{0, \ldots, 0}_{m-n})(q), \quad \forall q \in N .
$$

Então

$$
\mathrm{d} i\left(\left.\frac{\partial}{\partial x_{j}}\right|_{p}\right)=\left.\frac{\partial}{\partial y_{j}}\right|_{p}, \quad \forall p \in U, \quad \forall j \in\{1, \ldots, n\}
$$


O campo $X$ se escreve localmente como

$$
\left.X\right|_{U^{\prime}}=\sum_{j=1}^{m} a_{j} \frac{\partial}{\partial y_{j}},
$$

com $a_{j} \in \mathcal{C}^{\infty}\left(U^{\prime}\right)$ funções reais. Como $X$ é tangente a $N, a_{j}(p)=0, \quad \forall p \in$ $U, \quad \forall j: n<j \leq m$.

Assim,

$$
\begin{gathered}
(\mathrm{d} i)^{-1}\left(\left.X\right|_{p}\right)=(\mathrm{d} i)^{-1}\left(\left.\sum_{j=1}^{n} a_{j}(p) \frac{\partial}{\partial y_{j}}\right|_{p}\right)=\left.\sum_{j=1}^{n} a_{j}(p) \frac{\partial}{\partial x_{j}}\right|_{p}, \quad \forall p \in U . \\
\therefore(\mathrm{d} i)^{-1}\left(\left.X\right|_{p}\right)\left[\left.g\right|_{p}\right]=\left.\sum_{j=1}^{n} a_{j}(p) \frac{\partial}{\partial x_{j}}\right|_{p}\left[\left.g\right|_{p}\right], \quad \forall p \in U .
\end{gathered}
$$

Portanto a função $q \mapsto(\mathrm{d} i)^{-1}\left(\left.X\right|_{p}\right)\left[\left.g\right|_{q}\right]$ é igual, quando restrita a $U$, a $\sum_{j=1}^{n}\left(\left.a_{j}\right|_{U}\right) \partial g / \partial x_{j}$. Isto demonstra a suavidade e fica definido o campo $\left.X\right|_{N}$, com a seguinte fórmula em coordenadas locais:

$$
\left.\left(X \mid{ }_{N}\right)\right|_{U}=\left.\sum_{j=1}^{n} a_{j}\right|_{N} \frac{\partial}{\partial x_{j}}
$$

Seja $f \in \mathcal{C}^{\infty}(M)$. Mostremos que $\left(\left.X\right|_{N}\right)\left(\left.f\right|_{N}\right)=\left.(X f)\right|_{N}$. Dado $p \in N$, sejam $\vec{x}$ e $\vec{y}$ cartas locais de $N$ e $M$ definidas em $U$ e $U^{\prime}$ como foi feito acima, e escreva $\left.X\right|_{U^{\prime}}=\sum_{j=1}^{m} a_{j} \partial_{y_{j}}$, também como antes.

$$
\begin{aligned}
X f(p)=\left.\sum_{j=1}^{m} a_{j}(p) \frac{\partial f}{\partial y_{j}}\right|_{p}=\left.\sum_{j=1}^{n} a_{j}(p) \frac{\partial f}{\partial y_{j}}\right|_{p} & =\left.\sum_{j=1}^{n} a_{j}(p) \frac{\partial\left(\left.f\right|_{N}\right)}{\partial x_{j}}\right|_{p} \\
& =\left.X\right|_{N}\left(\left.f\right|_{N}\right)(p) .
\end{aligned}
$$

Unicidade: Seja $Y \in \mathfrak{X}(N)$ tal que $\forall f \in \mathcal{C}^{\infty}(M):\left.(X f)\right|_{N}=Y\left(\left.f\right|_{N}\right)$. Mostremos que $Y=\left.X\right|_{N}$. Isto é, mostremos que $\forall p \in N:\left.Y\right|_{p}=(\mathrm{d} i)^{-1}\left(\left.X\right|_{p}\right)$.

$$
\begin{gathered}
\begin{aligned}
& \mathrm{d} i\left(\left.Y\right|_{p}\right)\left[\left.f\right|_{p}\right]=\left.Y\right|_{p}\left[\left.(f \circ i)\right|_{p}\right]=\left.Y\right|_{p}\left[\left.\left(\left.f\right|_{N}\right)\right|_{p}\right]=Y\left(\left.f\right|_{N}\right)(p)=\left.(X f)\right|_{N}(p) \\
&= X f(p)=\left.X\right|_{p}\left[\left.f\right|_{p}\right], \quad \forall f \in \mathcal{C}^{\infty}(M) . \\
& \therefore \mathrm{d} i\left(\left.Y\right|_{p}\right)=\left.\left.X\right|_{p} \quad \therefore Y\right|_{p}=(\mathrm{d} i)^{-1}\left(\left.X\right|_{p}\right) .
\end{aligned}
\end{gathered}
$$




\subsubsection{Imagem de subvariedade por difeomorfismo}

Proposição 1.58. Seja $M^{\prime} \subset M$ uma subvariedade imersa de $M$ e $F$ : $M \rightarrow N$ um difeomorfismo. Então $F\left(M^{\prime}\right)$ é uma subvariedade imersa de $N$, $\left.F\right|_{M^{\prime} \rightarrow F\left(M^{\prime}\right)}$ é difeomorfismo entre $M^{\prime}$ e $F\left(M^{\prime}\right)$ e, para cada $p \in M^{\prime}$, o espaço tangente de $F\left(M^{\prime}\right)$ é dado por $\mathrm{T}_{F(p)} F\left(M^{\prime}\right)=\mathrm{d} F\left(\mathrm{~T}_{p} M^{\prime}\right)$.

Demonstração. Considere a restrição $\left.F\right|_{M^{\prime}} .\left.F\right|_{M^{\prime}}=F \circ i$, onde $i$ é a inclusão de $M^{\prime}$ em $M$. Assim, $\left.F\right|_{M^{\prime}}$ será suave e uma imersão (por ser composição de imersões). O resultado segue da proposição 1.38 (imagem de imersão injetiva é subvariedade) e da regra da cadeia (para calcular o diferencial de $F \circ i$ ). 


\section{Capítulo 2}

\section{Órbitas de Sussmann}

\subsection{Curvas integrais e fluxo}

\subsubsection{Curvas integrais}

Definição 2.1. Sejam $M$ uma variedade suave e $X \in \mathfrak{X}(M)$ um campo vetorial real em $M$.

(i) Uma curva $\alpha:(a, b) \rightarrow M$, com $a, b \in[-\infty, \infty], a<b$ é chamada de curva integral do campo $X$ quando $\alpha^{\prime}(t)=\left.X\right|_{\alpha(t)}, \forall t \in(a, b)$.

(ii) Quando $0 \in(a, b)$ e $p=\alpha(0)$, dizemos que a curva $\alpha$ está centrada em $p$.

(iii) Dizemos que a curva integral $\alpha$ é maximal quando não existir nenhuma outra curva integral $\beta:(c, d) \rightarrow M$ que seja extensão de $\alpha$, isto é, que tenha $(a, b) \subsetneq(c, d)$ e $\left.\beta\right|_{(a, b)}=\alpha$.

Observação 2.2. Estamos pedindo na definição que o campo vetorial seja real: pois a derivada de uma curva em uma variedade, $\alpha^{\prime}(t)$, é sempre um vetor tangente real.

Proposição 2.3 (Existência e Unicidade das Curvas Integrais). Sejam $M$ variedade suave, $X \in \mathfrak{X}(M)$ um campo real, $t_{0} \in \mathbb{R}$ e $p \in M$. Então existe $\delta>0$ tal que:

(i) $\forall T \in(0, \delta)$, existe uma única curva integral $\alpha:\left(t_{0}-T, t_{0}+T\right) \rightarrow M$ de $X \operatorname{com} \alpha\left(t_{0}\right)=p$.

(ii) Existe uma vizinhança aberta $V$ de $p$ tal que, para cada $p^{\prime} \in V, \forall T \in$ $(0, \delta)$, existe uma única curva integral $\alpha:\left(t_{0}-T, t_{0}+T\right) \rightarrow M$ de $X$ com $\alpha\left(t_{0}\right)=p^{\prime}$. 
Demonstração. Isto é uma aplicação do Teorema de Picard.

Corolário 2.4. Sejam $M$ uma variedade suave e $X \in \mathfrak{X}(M)$. Dado $p \in M$, existe uma única curva integral maximal de $X$ centrada em $p$.

Observação 2.5. Isto implica que uma curva integral não pode ter pontos críticos, a menos que ela seja constante.

Proposição 2.6 (Curva integral na subvariedade). Seja $X \in \mathfrak{X}(M)$ um campo vetorial em $M$ e $N$ uma subvariedade imersa de $M$, com $X$ tangente a $N$. Seja $\alpha:(a, b) \rightarrow N$ uma curva integral de $\left.X\right|_{N}$. Então a inclusão de $\alpha$ em $M$ é uma curva integral de $X$.

Demonstração. $(i \circ \alpha)^{\prime}(t)=\mathrm{d} i\left(\alpha^{\prime}(t)\right)=\left.X\right|_{\alpha(t)}$.

Exemplo 2.7. Em geral, a reparametrização de uma curva integral não é curva integral. Procuremos condições para que isto ocorra. Seja $\phi:(a, b) \rightarrow(c, d)$ um difeomorfismo entre intervalos e $\alpha:(a, b) \rightarrow M$ curva integral do campo $X$. Então

$$
\begin{gathered}
(\alpha \circ \phi)^{\prime}(t)\left[\left.f\right|_{\alpha(t)}\right]=(f \circ \alpha \circ \phi)^{\prime}(t)=((f \circ \alpha) \circ \phi)^{\prime}(t) \\
=\phi^{\prime}(t)(f \circ \alpha)^{\prime}(\phi(t))=\phi^{\prime}(t) \alpha^{\prime}(t)\left[\left.f\right|_{\alpha(t)}\right] \\
\therefore(\alpha \circ \phi)^{\prime}(t)=\phi^{\prime}(t) \alpha^{\prime}(\phi(t))=\left.\phi^{\prime}(t) X\right|_{\alpha \circ \phi(t)} .
\end{gathered}
$$

Assim, a reparametrização $\alpha \circ \phi$ só é uma curva integral de $X$ quando $\phi$ é uma translação:

$$
\begin{aligned}
\phi:(a, b) & \longrightarrow\left(a+t_{0}, b+t_{0}\right) \\
t & \longmapsto t+t_{0}
\end{aligned}
$$

Exemplo 2.8. Seja $M=\mathbb{R}^{2}$ com a estrutura diferenciável gerada pela carta global $\operatorname{Id}_{\mathbb{R}^{2}}=(x, y)$. Seja $X=\partial_{x}$.

Dado $p=\left(x_{0}, y_{0}\right)$, a curva integral maximal de $\mathrm{X}$ centrada em $p$ é $\alpha$ : $\mathbb{R} \rightarrow \mathbb{R}^{2}$ dada por $\alpha(t):=\left(x_{0}+t, y_{0}\right)$.

De fato, pois

$$
\alpha^{\prime}(t)=\left.\frac{\partial}{\partial x}\right|_{\left(x_{0}+t, y_{0}\right)}=\left.\frac{\partial}{\partial x}\right|_{\alpha(t)}
$$

e o domínio de $\alpha$ já é a reta inteira, não admitindo extensões.

Exemplo 2.9. Nem sempre a curva integral maximal pode ter como domínio a reta real inteira. Seja $M=(a, b), \operatorname{com}(a, b) \subset \mathbb{R},\left.\operatorname{Id}\right|_{(a, b)}=x$ carta global e $X=\mathrm{d} / \mathrm{d} x$. Seja $p=x_{0} \in(a, b)$. Neste caso, a curva integral maximal centrada em p será dada por $\alpha:\left(a-x_{0}, b-x_{0}\right) \rightarrow M$, com $\alpha(t)=x_{0}+t$. 
Exemplo 2.10. $M=\mathbb{R}^{2} \backslash\{0\},(x, y)=\operatorname{Id}_{\mathbb{R}^{2} \backslash\{0\}}$ carta global.

Considere os campos

$$
\frac{\partial}{\partial \theta}:=-y \frac{\partial}{\partial x}+x \frac{\partial}{\partial y} \quad \text { e } \quad \frac{\partial}{\partial r}:=\frac{x}{r} \frac{\partial}{\partial x}+\frac{y}{r} \frac{\partial}{\partial y}
$$

onde a função $r \in \mathcal{C}^{\infty}(M)$ é definida por $r:=\sqrt{x^{2}+y^{2}}$.

Dado o ponto $p=\left(r_{0} \cos \theta_{0}, r_{0} \operatorname{sen} \theta_{0}\right)$, as curvas integrais maximais de $\partial / \partial \theta$ e de $\partial / \partial r$ são, respectivamente:

$$
\begin{aligned}
\alpha: \mathbb{R} & \longrightarrow M \\
t & \longmapsto\left(r_{0} \cos \left(\theta_{0}+t\right), r_{0} \operatorname{sen}\left(\theta_{0}+t\right)\right)
\end{aligned}
$$

(rotação de $p_{0}$ em torno da origem com velocidade angular 1), e

$$
\begin{aligned}
\beta:\left(-r_{0}, \infty\right) & \longrightarrow M \\
t & \longmapsto\left(\left(r_{0}+t\right) \cos \theta_{0},\left(r_{0}+t\right) \operatorname{sen} \theta_{0}\right)
\end{aligned}
$$

(movimento linear de $p_{0}$ na direção radial, com velocidade 1 ).

\subsubsection{Fluxo}

Definição 2.11. Seja $M$ uma variedade suave e $X \in \mathfrak{X}(M)$ um campo vetorial real em $M$. Dado $p \in M$, seja $\alpha_{p}:\left(a_{p}, b_{p}\right) \rightarrow M$ a curva integral maximal de $X$ centrada em $p$. Para cada $t \in\left(a_{p}, b_{p}\right)$, denotaremos por $X_{t}(p)$ o ponto $\alpha_{p}(t)$. A aplicação $(p, t) \mapsto X_{t}(p)$ será chamada de fluxo do campo $X$.

Proposição 2.12 (Suavidade do Fluxo). O fluxo de um campo vetorial $X \in$ $\mathfrak{X}(M)$ é uma aplicação suave, definida em um subconjunto aberto de $M \times \mathbb{R}$.

Demonstração. Ver Sotomayor [7], capítulo VI, para uma prova em $\mathbb{R}^{n}$.

Proposição 2.13 (Propriedade de Grupo). Sejam $X \in \mathfrak{X}(M), t, s \in \mathbb{R} e$ $p \in M$. Se $X_{t}\left(X_{s}(p)\right)$ for definido, $X_{t+s}(p)$ também é definido e $X_{t}\left(X_{s}(p)\right)=$ $X_{t+s}(p)$.

Demonstração. Seja $\alpha:(a, b) \rightarrow M:=r \mapsto X_{r}(p)$ a curva integral maximal de $X$ centrada em $p$ e seja $\beta:(c, d) \rightarrow M:=r \mapsto X_{r}\left(X_{s}(p)\right)$ a curva integral maximal de $X$ centrada em $X_{s}(p)$. Por hipótese, $s \in(a, b)$ e $t \in(c, d)$.

Seja $\alpha_{s}:(a-s, b-s) \rightarrow M$ a curva $\alpha$ transladada:

$$
\alpha_{s}(r)=\alpha(r+s) .
$$


Sabemos (exemplo 2.7) que a translação de uma curva integral também é uma curva integral. É fácil ver que a translação de uma curva integral maximal é outra curva integral maximal. Assim, $\alpha_{s}$ é uma curva integral maximal de $X$. Como $\alpha_{s}(0)=\alpha(s)=X_{s}(p)$, temos que $\alpha_{s}=\beta$ e portanto $(c, d)=$ $(a-s, b-s)$.

Assim, $t \in(a-s, b-s)$ e $\alpha_{s}(t)=X_{s+t}(p)=\beta(t)=X_{t}\left(X_{s}(p)\right)$.

Exemplo 2.14. $X=\partial_{x}$ em $\mathbb{R}^{2}$, com Id $\left.\right|_{\mathbb{R}^{2}}=(x, y)$. Então, para cada $p \in \mathbb{R}^{2}$, e para cada $t \in \mathbb{R}$

$$
X_{t}(p)=(x(p)+t, y(p)) .
$$

Exemplo 2.15. $M=(a, b) \subset \mathbb{R}$, com a estrutura diferenciável induzida pela carta global $x=\operatorname{Id}_{\mathbb{R}} ; X=\mathrm{d} / \mathrm{d} x$. Então, dado $p \in \mathbb{R}$,

$$
X_{t}(p)=p+t \text {, desde que } p+t \in(a, b) .
$$

Neste caso, o domínio do fluxo é $\left\{(p, t) \in \mathbb{R}^{2} \mid p \in(a, b)\right.$ e $\left.p+t \in(a, b)\right\}$.

Exemplo 2.16. $M=\mathbb{R}^{2}$,

$$
X=\frac{\partial}{\partial \theta}:=-y \frac{\partial}{\partial x}+x \frac{\partial}{\partial y} .
$$

Então

$$
X_{t}(p)=(x(p) \cos t-y(p) \operatorname{sen} t, x(p) \operatorname{sen} t+y(p) \cos t),
$$

definido para todo $t$.

\subsection{Difeomorfismos locais}

\subsubsection{Definição e operações}

Definição 2.17. (i) Um difeomorfismo local na variedade suave $M$ é um difeomorfismo entre dois abertos de $M$. A aplicação vazia, cujos domínio e contra-domínio são o subconjunto vazio de $M$, é considerada também como um difeomorfismo local.

(ii) Dados dois difeomorfismos locais $\phi: U \subset M \mapsto V \subset M$ e $\psi: U^{\prime} \subset$ $M \mapsto V^{\prime} \subset M$, definimos o seu produto como sendo o seguinte difeomorfismo local, potencialmente vazio:

$$
\begin{array}{cl}
\phi \psi: \psi^{-1}\left(V^{\prime} \cap U\right) & \longrightarrow \phi\left(V^{\prime} \cap U\right) \\
p & \longmapsto \phi(\psi(p))
\end{array}
$$

(iii) Definimos o inverso de um difeomorfismo local (notação $\phi^{-1}$ ) como sendo a sua função inversa. 


\section{Propriedades das operações}

Proposição 2.18. (i) O produto de difeomorfismos locais é associativo.

(ii) Dados $\phi_{1}, \phi_{2}$ difeomorfismos locais, $\left(\phi_{1} \phi_{2}\right)^{-1}=\phi_{2}^{-1} \phi_{1}^{-1}$.

Demonstração. Associatividade do produto: Sejam

$$
\phi_{1}: U_{1} \rightarrow V_{1}, \quad \phi_{2}: U_{2} \rightarrow V_{2} \quad \text { e } \quad \phi_{3}: U_{3} \rightarrow V_{3} .
$$

Mostremos que o produto de difeomorfismos locais é associativo. Isto é, que

$$
\phi_{1}\left(\phi_{2} \phi_{3}\right)=\left(\phi_{1} \phi_{2}\right) \phi_{3}
$$

Para isto, precisamos identificar o domínio e o contra-domínio destas aplicações.

$$
\begin{aligned}
\phi_{2} \phi_{3}: \phi_{3}^{-1}\left(V_{3} \cap U_{2}\right) & \longrightarrow \phi_{2}\left(V_{3} \cap U_{2}\right) \\
p & \longmapsto \phi_{2}\left(\phi_{3}(p)\right) \\
\phi_{1}\left(\phi_{2} \phi_{3}\right): \quad\left(\phi_{2} \phi_{3}\right)^{-1}\left(\phi_{2}\left(V_{3} \cap U_{2}\right) \cap U_{1}\right) & \longrightarrow \phi_{1}\left(\phi_{2}\left(V_{3} \cap U_{2}\right) \cap U_{1}\right) \\
p & \longmapsto \phi_{1}\left(\phi_{2}\left(\phi_{3}(p)\right)\right) \\
\phi_{1} \phi_{2}: \phi_{2}^{-1}\left(U_{1} \cap V_{2}\right) & \longrightarrow \phi_{1}\left(U_{1} \cap V_{2}\right) \\
p & \longmapsto \phi_{1}\left(\phi_{2}(p)\right) \\
\left(\phi_{1} \phi_{2}\right) \phi_{3}: \quad \phi_{3}^{-1}\left(V_{3} \cap \phi_{2}^{-1}\left(U_{1} \cap V_{2}\right)\right) & \longrightarrow \phi_{1} \phi_{2}\left(V_{3} \cap \phi_{2}^{-1}\left(U_{1} \cap V_{2}\right)\right) \\
p & \longmapsto \phi_{1}\left(\phi_{2}\left(\phi_{3}(p)\right)\right)
\end{aligned}
$$

Assim, temos que mostrar as seguintes igualdades de conjuntos:

$$
\begin{aligned}
\phi_{3}^{-1}\left(V_{3} \cap \phi_{2}^{-1}\left(U_{1} \cap V_{2}\right)\right) & =\left(\phi_{2} \phi_{3}\right)^{-1}\left(\phi_{2}\left(V_{3} \cap U_{2}\right) \cap U_{1}\right) \mathrm{e} \\
\phi_{1} \phi_{2}\left(V_{3} \cap \phi_{2}^{-1}\left(U_{1} \cap V_{2}\right)\right) & =\phi_{1}\left(\phi_{2}\left(V_{3} \cap U_{2}\right) \cap U_{1}\right) .
\end{aligned}
$$

Demonstração de (2.19):

$$
\begin{gathered}
\left(\phi_{2} \phi_{3}\right)^{-1}\left(\phi_{2}\left(V_{3} \cap U_{2}\right) \cap U_{1}\right) \\
=\left\{p \in \phi_{3}^{-1}\left(V_{3} \cap U_{2}\right) \mid \phi_{2}\left(\phi_{3}(p)\right) \in \phi_{2}\left(V_{3} \cap U_{2}\right) \cap U_{1}\right\} \\
=\left\{p \in \phi_{3}^{-1}\left(V_{3} \cap U_{2}\right) \mid \phi_{3}(p) \in\left(V_{3} \cap U_{2}\right) \cap \phi_{2}^{-1}\left(U_{1} \cap V_{2}\right)\right\} \\
=\left\{p \in U_{3} \mid \phi_{3}(p) \in\left(V_{3} \cap U_{2}\right) \cap \phi_{2}^{-1}\left(U_{1} \cap V_{2}\right)\right\} \\
=\phi_{3}^{-1}\left(V_{3} \cap \phi_{2}^{-1}\left(U_{1} \cap V_{2}\right)\right) .
\end{gathered}
$$


Demonstração de (2.20):

$$
\begin{aligned}
\phi_{1} \phi_{2}\left(V_{3} \cap \phi_{2}^{-1}\left(U_{1} \cap V_{2}\right)\right)=\left\{\phi_{1}\left(\phi_{2}(p)\right) \mid p \in V_{3} \cap \phi_{2}^{-1}\left(U_{1} \cap V_{2}\right)\right\} \\
\quad=\left\{\phi_{1}(q) \mid q \in \phi_{2}\left(V_{3} \cap U_{2}\right) \cap\left(U_{1} \cap V_{2}\right)\right\} \\
=\left\{\phi_{1}(q) \mid q \in \phi_{2}\left(V_{3} \cap U_{2}\right) \cap U_{1}\right\}=\phi_{1}\left(\phi_{2}\left(V_{3} \cap U_{2}\right) \cap U_{1}\right) .
\end{aligned}
$$

Inversão do produto:

$$
\begin{aligned}
\left(\phi_{1} \phi_{2}\right)^{-1}: \phi_{1}\left(V_{2} \cap U_{1}\right) & \longrightarrow \phi_{2}^{-1}\left(V_{2} \cap U_{1}\right) \\
p & \longmapsto \phi_{2}^{-1}\left(\phi_{1}^{-1}(p)\right) \\
\phi_{2}^{-1} \phi_{1}^{-1}:\left(\phi_{1}^{-1}\right)^{-1}\left(U_{1} \cap V_{2}\right) & \longrightarrow \phi_{2}^{-1}\left(V_{2} \cap U_{1}\right) \\
p & \longmapsto \phi_{2}^{-1}\left(\phi_{1}^{-1}(p)\right) .
\end{aligned}
$$

$\operatorname{Como}\left(\phi_{1}^{-1}\right)^{-1}=\phi_{1},\left(\phi_{1} \phi_{2}\right)^{-1}=\phi_{2}^{-1} \phi_{1}^{-1}$.

\subsubsection{As aplicações $X_{t}$}

Definição 2.21. Dado um $t \in \mathbb{R}$ fixo, seja $\Omega_{t}$ o conjunto de todos os pontos $p \in M$ tais que $X_{t}(p)$ é definido. $\Omega_{t}$ é aberto (pois o domínio do fluxo é aberto, pela proposição 2.12) assim definimos a aplição:

$$
\begin{aligned}
X_{t}: \Omega_{t} & \longrightarrow X_{t}\left(\Omega_{t}\right) \subset M \\
p & \longmapsto X_{t}(p)
\end{aligned}
$$

Observação 2.22. $X_{t}$ é um difeomorfismo local: $X_{t}$ tem como função inversa $X_{-t}$. Ambas sao suaves, em virtude da proposição 2.12. Assim, $X_{t}$ é um difeomorfismo entre os abertos de $\Omega_{t}$ e $\Omega_{-t}$ de $M$.

\subsubsection{Grupos de difeomorfismos locais}

Definição 2.23. Um grupo de difeomorfismos locais na variedade suave $M$ é um conjunto de difeomorfismos locais que é fechado nas operações produto e inversão.

Observação 2.24. Um grupo de difeomorfismos locais pode não ser um grupo no sentido algébrico do termo. Por exemplo, seja $G$ o conjunto de todos os difeomorfismos locais de $M$. G é um grupo de difeomorfismos locais. O elemento neutro de $G$, se $G$ fosse um grupo no sentido algébrico, seria a identidade Id $: M \rightarrow M$.

Se $\phi: U \rightarrow V$ é um difeomorfismo local, $\phi \phi^{-1}=\left.\mathrm{Id}\right|_{U}$, a restrição de $\mathrm{Id}$ ao aberto $U$, que só coincide com Id quando $U=M$. 
Exemplo 2.25. Dado um campo real $X \in \mathfrak{X}(M)$, suponha que as curvas integrais maximais de $X$ tenham como domínio toda a reta real. Então as aplicações $X_{t}$ são definidas em toda a variedade $M$ para todo $t \in \mathbb{R}$. Considere o conjunto $G=\left\{X_{t} \mid t \in \mathbb{R}\right\}$. Sejam $X_{t}$ e $X_{s}$ elementos de $G$. Pela proposição 2.13, $X_{t} X_{s}=X_{t+s}$ e $X_{t}^{-1}=X_{-t}$. Assim, $G$ é um grupo de difeomorfismos locais - e também, neste caso, um grupo no sentido algébrico do termo.

\subsection{Famílias de campos vetoriais e órbitas}

\subsubsection{Famílias de campos vetoriais}

Definição 2.26. (i) Uma família de campos vetoriais $D$ em $M$ é um conjunto de campos vetoriais (reais) definidos em abertos da variedade $M$.

(ii) Diremos que a família de campos vetoriais $D$ é definida em todo lugar quando, para todo ponto $p \in M, p$ está no domínio de algum dos campos vetoriais de $D$.

Seja $D$ uma família de campos vetoriais em $M$.

Definição 2.27. O grupo gerado por $D, G_{D}$, é o menor grupo de difeomorfismos locais que contém todas as aplicações da forma $X_{t}$, com $X \in D$ e $t \in \mathbb{R}$.

Sejam $n \in \mathbb{N}$ um número natural, $\xi=\left(X^{1}, X^{2}, \ldots, X^{n}\right) \in D^{n}$ uma $n$-upla de campos vetoriais da família $D, T=\left(t_{1}, t_{2}, \ldots, t_{n}\right) \in \mathbb{R}^{n}$. Denotaremos por $\xi_{T}$ o difeomorfismo local $X_{t_{1}}^{1} X_{t_{2}}^{2} \cdots X_{t_{n}}^{n}$.

Proposição 2.28. $G_{D}=C$, onde

$$
C=\left\{\xi_{T} \mid \xi \in D^{n}, T \in \mathbb{R}^{n}, n \in \mathbb{N} \backslash\{0\}\right\}
$$

Demonstração. Afirmação: $C \subset G_{D}$.

De fato, dado $\xi_{T}:=X_{t_{1}}^{1} \cdots X_{t_{N}}^{n} \in C, \xi_{T}$ é formado usando-se apenas a operação de composição a partir dos elementos $X_{t_{1}}^{1}, \ldots, X_{t_{n}}^{n}$ e portanto está em todos os grupos que os contém. Em particular, $\xi_{T} \in G_{D}$.

Afirmação: $C$ é um grupo de difeomorfismos locais.

De fato: dados

$$
\xi_{T}:=X_{t_{1}}^{1} \cdots X_{t_{n}}^{n} \text { e } \eta_{S}:=Y_{s_{1}}^{1} \cdots Y_{s_{m}}^{m} \in C,
$$

temos

$$
\xi_{T} \eta_{S}=X_{t_{1}}^{1} \cdots X_{t_{n}}^{n} Y_{s_{1}}^{1} \cdots Y_{s_{m}}^{n} \stackrel{\text { notacão }}{=}(\xi \eta)_{T S} \in C
$$


e

$$
\left(\xi_{T}\right)^{-1}=X_{-t_{n}}^{n} \cdots X_{-t_{1}}^{1} \stackrel{\text { notacão }}{=} \hat{\xi}_{-\hat{T}} \in C
$$

Portanto, $C=G_{D}$.

Exemplo 2.29. $M=\mathbb{R}^{3}$, Id $: \mathbb{R}^{3} \rightarrow \mathbb{R}^{3}=(x, y, z), D=\{X, Y\}$, com $X=\partial_{x}$ e $Y=\partial_{y}$.

$$
X_{t}, Y_{t}: \mathbb{R}^{3} \rightarrow \mathbb{R}^{3} \quad X_{t}=(x+t, y, z) \quad Y_{t}=(x, y+t, z)
$$

Neste caso $G_{D}=\left\{X_{t_{1}} Y_{t_{2}} \mid t_{1}, t_{2} \in \mathbb{R}\right\}$

Para verificar este exemplo, basta mostrar que o conjunto acima é um grupo, o que é consequência de as aplicações da forma $X_{t_{1}}$ e $Y_{t_{2}}$ comutarem.

\subsubsection{Definição das $D$-órbitas}

Definição 2.30. Seja $D$ uma família de campos vetoriais definida em todo lugar. Dizemos que $p \sim q$ se $\exists \phi \in G_{D}: \phi(p)=q$. A relação $\sim$ é de equivalência. As órbitas da família $D$ ou $D$-órbitas são as classes de equivalência de $\sim$.

Observação 2.31. Mostremos que , como definida acima, é mesmo uma relação de equivalência.

$p \sim p$ : Como $D$ é definido em todo lugar, existe $X \in D, X \in \mathfrak{X}(\Omega)$ com $p \in \Omega \subset M$. A aplicação $X_{0}$ é a identidade em $\Omega$, então $p=X_{0}(p)$.

$p \sim q \Rightarrow q \sim p$ : Seja $\phi \in G_{D}$ tal que $\phi(p)=q$. Então $\phi^{-1}(q)=p$ e $\phi^{-1} \in G_{D}$.

$p \sim q$ e $q \sim r \Rightarrow p \sim r$ : Sejam $\phi, \psi \in G_{D}$ tais que $\phi(p)=q$ e $\psi(q)=r$. Então $\psi \phi(p)=r$, e $\psi \phi \in G_{D}$.

\subsubsection{Definição por curvas integrais por partes}

Definição 2.32. Seja $D$ uma família de campos vetoriais definida em todo lugar na variedade $M$. Uma curva integral por partes de $D$ é um caminho contínuo $\alpha:[a, b] \rightarrow M$ tal que existe uma partição $a=t_{0}<t_{1}<\cdots<t_{n}=$ $b$ de $[a, b]$ e existem campos vetoriais $X^{1}, \ldots, X^{n} \in D$ tais que $\left.\alpha\right|_{\left(t_{j-1}, t_{j}\right)}$ é curva integral de $X^{j}$ ou de $-X^{j}$ para todo $j \in 1,2, \ldots, n$.

Proposição 2.33. Seja $\approx$ a relação, nos pontos de $M$, definida pela regra: $p \approx q \Leftrightarrow \exists \alpha:[a, b] \rightarrow M$, curva integral por partes de $D$, tal que $\alpha(a)=$ p e $\alpha(b)=q$.

Então $\approx$ é uma relação de equivalência que coincide com $\sim$, da definição 2.30. 
Demonstração. $p \approx q \Rightarrow q \approx p$ : Pois o caminho inverso de uma curva integral por partes de $D$ ainda é uma curva integral por partes de $D$.

$p \approx q$ e $q \approx r \Rightarrow p \approx r$ : Pois a concatenação de duas curvas integrais por partes ainda é uma curva integral por partes.

$p \approx p$ : como $D$ é definida em todo lugar, há um campo $X \in D$ que é definido em um aberto contendo $p$. Usando a curva integral de $X$ centrada em $p$, podemos encontrar $q \in M$ (potencialmente igual) tal que $p \approx q$. Como $p \approx q$ e $q \approx p$, temos $p \approx p$.

Assim, $\approx$ é uma relação de equivalência.

Se $q=X_{t}(p), X \in D, t \in \mathbb{R}$, então podemos ligar $p$ e $q$ por um segmento de curva integral, e portanto $q \approx p$. Mais geralmente, se $q=\xi_{T}(p)$, com $\xi_{T}=$ $\left(X^{1}\right)_{t_{1}} \cdots\left(X^{n}\right)_{t_{n}}$, então podemos fazer indução em $n$ e usar a transitividade de $\approx$ para concluir que $p \approx q$. Assim, $p \approx q$ sempre que $p \sim q$.

Por outro lado, suponha que $p$ e $q$ se ligam por uma curva integral por partes de um só segmento, isto é, existe $\alpha:[a, b] \rightarrow M$ tal que $\alpha(a)=p$, $\alpha(b)=q$ e $\left.\alpha\right|_{(a, b)}$ é curva integral de algum campo $X \in D$ (se $\left.\alpha\right|_{(a, b)}$ for curva integral de $-X$, podemos trocar $p$ e $q$ sem perda de generalidade). Ainda, podemos supor que $a=0$.

Como existem os limites laterais de $\left.\alpha\right|_{(0, b)}$, podemos estendê-la pelos dois lados (isto é consequência da proposição 2.4). A extensão maximal de $\left.\alpha\right|_{(0, b)}$ coincide com $X_{t}(p)$, e passa por $q$. Portanto, $p \sim q$. Por indução, e novamente usando a transitividade da relação $\approx$, se $p$ se liga a $q$ por uma curva integral por partes de $n$ segmentos, $p \sim q$.

Assim, são equivalentes: $p \approx q$ e $p \sim q$.

Corolário 2.34. As D-órbitas coincidem com as classes de equivalência de ح. Assim, podemos usar a relação "estão ligados por uma curva integral por partes" nos pontos de $M$ como uma maneira alternativa de definir as D-órbitas.

Exemplo 2.35. (i) $M=\mathbb{R}^{3}, D=\left\{\partial_{x}, \partial_{y}\right\}$. Então as $D$-órbitas serão os planos horizontais.

(ii) $M=\mathbb{R}^{3}, D=\left\{\partial_{z}, \partial_{\theta}\right\}$, onde $\partial_{\theta}:=-y \partial_{x}+x \partial_{y}$. Neste caso, as $D$-órbitas são os cilindros (de base circular) em torno do eixo $z$ e o próprio eixo $z$.

(iii) $M=\mathbb{R}^{3}, D=\left\{X^{1}, X^{2}\right\}$, com

$$
X^{1}=\frac{\partial}{\partial x} \text { e } X^{2}=\frac{\partial}{\partial y}+x \frac{\partial}{\partial z} .
$$

Então a única órbita é $\mathbb{R}^{3}$. 
O primeiro e o segundo caso são fáceis de se ver, usando-se que as curvas integrais dos campos em questão são retas e círculos. Para o terceiro exemplo, dado $p=\left(x_{0}, y_{0}, z_{0}\right) \in \mathbb{R}^{3}$, as seguintes ligações podem ser feitas por curvas integrais dos campos indicados:

$$
\left(x_{0}, y_{0}, z_{0}\right) \stackrel{X^{1}}{\approx}\left(0, y_{0}, z_{0}\right) \stackrel{X^{2}}{\approx}\left(0, z_{0}, z_{0}\right) \stackrel{X^{1}}{\approx}\left(1, z_{0}, z_{0}\right) \stackrel{X^{2}}{\approx}(1,0,0)
$$

\subsubsection{As aplicações $\rho_{\xi, p}$}

Definição 2.36. Para cada $n$-upla $\xi \in D^{n}$, e para cada $p \in M$, seja $\Omega_{\xi, p} \mathrm{O}$ conjunto de todos os $T \in \mathbb{R}^{n}$ tais que $\xi_{T}(p)$ é definido. Para cada $n \in \mathbb{N}$, $\xi \in D^{n}$, definimos a aplicação:

$$
\begin{aligned}
\rho_{\xi, p}: \Omega_{\xi, p} \subset \mathbb{R}^{n} & \longrightarrow M \\
T & \longmapsto \xi_{T}(p) .
\end{aligned}
$$

Proposição 2.37. O conjunto $\Omega_{\xi, p}$ da definição acima é aberto em $\mathbb{R}^{n}$.

Demonstração. Seja $\xi=\left(X^{1}, \ldots, X^{n}\right)$. Façamos uma indução finita em $n$ :

Se $n=1$ : então $\Omega_{\xi, p}$ é o domínio da curva integral maximal centrada em $p$, que é aberto.

Se $n>1$, e supondo que o teorema vale para $n-1$ : Seja $T=\left(t_{1}, \ldots, t_{n}\right) \in$ $\Omega_{\xi, p}$.

$$
\xi_{T}(p)=X_{t_{1}}^{1}\left(\eta_{S}(p)\right)
$$

onde $\eta=\left(X^{2}, \ldots, X^{n}\right)$ e $S=\left(t_{2}, \ldots, t_{n}\right)$

Por indução, existe uma vizinhança (aberta) $V^{\prime} \subset \mathbb{R}^{n-1}$ de $S$ tal que $\eta_{R}(p)$ é definido para todo $R \in V^{\prime}$. Pela continuidade do fluxo, se $W$ é uma vizinhança de $\eta_{S}(p)$, podemos restringir $V^{\prime}$ de forma que $\eta_{R}(p) \in W, \forall R \in V^{\prime}$. Por 2.3, podemos escolher a vizinhança $W$ de tal forma que as curvas integrais centradas em $p^{\prime}=\eta_{R}(p) \in W$, dadas por $X_{t_{1}}^{1}\left(\eta_{R}(p)\right)$ na variável $t_{1}$, são definidas para todo $t_{1} \in(-r, r)$, onde $r>0$ pode ser escolhido uniformemente. Assim, o aberto $(-r, r) \times V^{\prime}$ está contido em $\Omega_{\xi, p}$, e é vizinhança de $T$.

Proposição 2.38. As aplicações $\rho_{\xi, p}$ são suaves.

Demonstração. Isto é consequência da suavidade do fluxo. Dado $\xi \in D^{n}$, façamos indução em $n$. Se $n=1, \xi=(X)$ e portanto $\rho_{\xi, p}(t)=X_{t}(p)=$ $\Phi(p, t)$, onde $\Phi$ é o fluxo de $X$, que sabemos ser suave.

Se $n>1$, e usando a hipótese de indução, escrevamos $\xi=(X) \eta$, onde $X \in D, \eta \in D^{n-1} \cdot \rho_{\xi, p}\left(t_{1}, \ldots, t_{n}\right)=\Phi\left(\rho_{\eta, p}\left(t_{2}, \ldots, t_{n}\right), t_{1}\right)$, onde $\Phi$ é o fluxo de $X$. Como $\rho_{\eta, p}$ e $\Phi$ são ambas suaves, $\rho_{\xi, p}$ é suave. 
Observação 2.39. Sejam $p$ um ponto da variedade suave $M$ e $D$ uma família definida em todo lugar de campos vetoriais em $M$. A $D$-órbita do ponto $p$ é dada pelo conjunto:

$$
S_{p}:=\bigcup_{n \in \mathbb{N}}\left\{\xi_{T}(p) \mid \xi \in D^{n}, T \in \mathbb{R}^{n} \text { e } p \in \operatorname{Dom}\left(\xi_{T}\right)\right\} .
$$

Com a definição das aplicações $\rho_{\xi, p}$, a $D$-órbita de um ponto $p \in M$ pode ser escrita como:

$$
S_{p}:=\bigcup_{n \in \mathbb{N}}\left\{\rho_{\xi, p}(T) \mid \xi \in D^{n}, T \in \Omega_{\xi, p}\right\}=\bigcup_{n \in \mathbb{N}} \bigcup_{\xi \in D^{n}} \operatorname{Imagem}\left(\rho_{\xi, p}\right) .
$$

\subsubsection{Topologia da $D$-órbita}

Definição 2.40. A topologia da $D$-órbita $S_{p}$ de um ponto $p$ é a topologia co-induzida pelas aplicações $\rho_{\xi, p}$, isto é:

$$
\tau_{p}=\left\{U \subset S_{p} \mid \rho_{\xi, p}^{-1}(U) \text { é aberto em } \Omega_{\xi, p}\right\}
$$

Proposição 2.41. A topologia da D-órbita é bem-definida. Isto é, se $p$ e q estão na mesma órbita, as topologias $\tau_{p}$ e $\tau_{q}$ são iguais.

Demonstração. Sejam $p$ e $q$ na mesma órbita. Seja $U \in \tau_{p}$. Mostremos que $U \in \tau_{q}$. Dados $n \in \mathbb{N}, \xi \in D^{n}$, queremos mostrar que $\rho_{\xi, q}^{-1}(U)$ é aberto em $\Omega_{\xi, q}$. Como $p$ e $q$ estão na mesma órbita, existem $m \in \mathbb{N}, \eta \in D^{m}$ e $T_{0} \in \Omega_{\eta, p}$ tais que $\eta_{T_{0}}(p)=q$.

Dado $T=\left(t_{1}, \ldots, t_{n}\right) \in \Omega_{\xi, q}$,

$$
\rho_{\xi, q}(T)=\xi_{T}(q)=\xi_{T}\left(\eta_{T_{0}}(p)\right)=(\xi \eta)_{T T_{0}}(p)=\rho_{\xi \eta, p}\left(T T_{0}\right) .
$$
$T T_{0}$.

Portanto, $\rho_{\xi, q}=\rho_{\xi \eta, p} \circ C$, onde $C: \Omega_{\xi, q} \rightarrow \Omega_{\xi \eta, p}$ é a concatenação $C(T)=$

Assim, $\rho_{\xi, q}^{-1}(U)=C^{-1}\left(\rho_{\xi \eta, p}^{-1}(U)\right)$. Pela continuidade da concatenação $C$, obtemos o resultado desejado.

Proposição 2.42. (i) A topologia das D-órbitas é mais fina que a topologia do subespaço.

(ii) As órbitas, dotadas de suas topologias, são conexas.

Demonstração. (i) Seja $S_{p}$ a $D$-órbita de $p$ na variedade $M$. Se $U \subset M$ é um aberto de $M$, queremos mostrar que $U \cap S_{p}$ é aberto na topologia da órbita. Mas isto é consequência de as aplicações $\rho_{\xi, p}: \Omega_{\xi, p} \rightarrow M$ serem contínuas: como a imagem destas aplicações está contida em $S_{p}$, temos $\rho_{\xi, p}^{-1}\left(U \cap S_{p}\right)=\rho_{\xi, p}^{-1}(U)$, que é aberto. 
(ii) Seja $\alpha:(a, b) \rightarrow S_{p}$ tal que $\left.\alpha\right|_{(a, b) \rightarrow M}$ é uma curva integral de algum $X \in D$. Mostremos que $\alpha$ é contínua (em relação à topologia de $S_{p}$ ).

Podemos supor, sem perda de generalidade, que $\alpha$ é maximal — pois toda curva integral é restrição de alguma curva integral maximal, e a restrição preserva continuidade. Ainda podemos supor, sem perda de generalidade, que $0 \in(a, b)$, pois a translação de uma curva preserva continuidade.

Seja $q=\alpha(0)$. Assim, $\alpha$ coincide com a função $t \mapsto X_{t}(q)=\rho_{\xi_{0}, q}$, onde $\xi_{0}=(X)$. Pela proposição 2.41,

$$
\tau_{p}=\tau_{q}=\left\{U \subset S_{q} \mid \rho_{\xi, q}^{-1}(U) \text { é aberto, } \forall \xi \in D^{n}, \forall T \in \mathbb{R}^{n}, \forall n \in \mathbb{N}\right\} .
$$

Assim, se $U \in \tau_{p}, \alpha^{-1}(U)=\rho_{\xi_{0}, q}^{-1}(U)$ é aberto em $\Omega_{\xi_{0}, q}=(a, b)$. Ou seja, $\alpha$ é contínua.

Como as curvas integrais em $S_{p}$ são contínuas, e todos pontos de $S_{p}$ podem ser ligados por uma concatenação de segmentos de curvas integrais, concluímos que $S_{p}$ é conexa por caminhos - e, portanto, conexa.

Observação 2.43. Como a topologia de uma órbita é mais fina do que a sua topologia como subespaço, a órbita também será conexa como subespaço de $M$.

Observação 2.44. A topologia da D-órbita, em geral, não coincide com a topologia co-induzida por todas as curvas integrais de elementos de $D$. Em $\mathbb{R}^{2}$ com as coordenadas usuais, considere $D=\left\{\partial_{x}, \partial_{y}\right\}$. A única $D$-órbita é $\mathbb{R}^{2}$, e sua topologia será a topologia usual do plano (o que é facilmente demonstrado se usarmos o teorema 2.73 e a proposição 1.45$)$.

O conjunto

$$
W=\left\{p \in \mathbb{R}^{2} \mid\left(x^{2}+y^{2}\right)(p)<1\right\} \backslash\left\{\left(\frac{1}{n}, \frac{1}{n}\right) \mid n \in \mathbb{N}^{*}\right\} .
$$

é aberto na topologia co-induzida pelas curvas integrais de $\partial_{x}$ e $\partial_{y}$ : pois, dado $p \in W$, sempre podemos mover $p$ um pouco nas direções vertical e horizontal. Mas $W$ não é aberto na topologia usual do plano, pois 0 não é ponto interior de $W$.

\subsection{Distribuições}

Definição 2.45. Uma distribuição $\Delta$ é uma aplicação que associa a cada ponto $p \in M$ da variedade $M$ um subespaço vetorial $\Delta(p) \subset T_{p} M$ do espaço tangente de $M$ em $p$. 
Definição 2.46. (i) Dada uma família $D$ de campos vetoriais definida em todo lugar na variedade $M$, a distribuição gerada por $D, \Delta_{D}$, é a distribuição que associa a cada $p \in M$ o subespaço vetorial gerado pelos campos de $D$ que são definidos em $p$, isto é:

$$
\Delta_{D}(p)=\left\langle\left\{\left.X\right|_{p} \mid X \in D, X \text { é definido em } p\right\}\right\rangle
$$

(ii) Uma distribuição $\Delta$ que pode ser gerada por uma alguma família $D$ de campos vetoriais (suaves) é chamada de distribuição suave.

Definição 2.47. Dizemos que um campo vetorial $X \in \mathfrak{X}(\Omega)$, com $\Omega$ um aberto de $M$, pertence à distribuição $\Delta$ (notação $X \in \Delta$ ) se, para cada $p \in \Omega,\left.X\right|_{p} \in \Delta(p)$. A família de todos os campos vetoriais que pertencem a uma distribuição $\Delta$ é denotada $D_{\Delta}$.

Exemplo 2.48. Uma distribuição que não é suave: em $M=\mathbb{R}^{2}$ :

$$
\Delta(p)=\left\{\begin{array}{l}
\frac{\partial}{\partial x}, \text { se } x(p) \text { e } y(p) \text { são ambos racionais; } \\
\frac{\partial}{\partial y}, \text { se } x(p) \text { é irracional ou se } y(p) \text { é irracional. }
\end{array}\right.
$$

\subsubsection{Subvariedades integrais}

Definição 2.49. (i) Uma subvariedade integral da distribuição $\Delta$ é uma subvariedade imersa $N \subset M$ cujo espaço tangente coincide com $\Delta$ em cada ponto.

(ii) Uma distribuição $\Delta$ tem a propriedade das subvariedades integrais quando, para cada $p \in M$, existe uma subvariedade integral de $\Delta$ passando por $p$.

(iii) Uma subvariedade integral maximal da distribuição $\Delta$ é uma subvariedade integral $N$ de $\Delta$ tal que cada subvariedade integral conexa de $\Delta$ que intercepta $N$ é uma subvariedade aberta de $N$.

(iv) Uma distribuição $\Delta$ tem a propriedade das subvariedades integrais maximais quando, para cada $p \in M$, existe uma subvariedade integral maximal de $\Delta$ passando por $p$.

Proposição 2.50. Sejam $X^{1}, \ldots, X^{n}$ campos vetoriais na variedade $M$ que são tangentes à subvariedade $N \subset M$. Sejam

$$
\xi=\left(X^{1}, \ldots, X^{n}\right) \quad \text { e }\left.\xi\right|_{N}=\left(\left.X^{1}\right|_{N}, \ldots,\left.X^{n}\right|_{N}\right) .
$$

$\operatorname{Seja} T=\left(t_{1}, \ldots, t_{n}\right) \in \Omega_{\left.\xi\right|_{N}, p}$ Então $T \in \Omega_{\xi, p}$ e $\left(\left.\xi\right|_{N}\right)_{T}(p)=\xi_{T}(p)$. 
Demonstração. Se $n=1$ :

Então $\xi=(X), \xi_{T}=X_{t_{1}},\left(\left.\xi\right|_{N}\right)_{T}=\left(\left.X\right|_{N}\right)_{t_{1}}$. Seja $\alpha:(a, b) \rightarrow N$ a curva integral maximal de $\left.X\right|_{N}$ centrada em $p$, definida na subvariedade $N$. A inclusão de $\alpha$ em $M$ é uma curva integral de $X$ em $M$ centrada em $p$. Assim, dado $t \in(a, b), X_{t}(p)=(i \circ \alpha)(t)=\left(\left.X\right|_{N}\right)_{t}(p)$. Portanto, $X_{t_{1}}(p)=\left(\left.X\right|_{N}\right)_{t_{1}}(p)$.

Se $n>1$ e supondo a hipótese de indução:

Então $\xi=\eta \psi, \operatorname{com} \eta=\left(X_{1}\right)$ e $\psi=\left(X_{2}, \ldots, X_{n}\right) .\left.\quad \xi\right|_{N}=\left(\left.\eta\right|_{N}\right)\left(\left.\psi\right|_{N}\right)$. Seja $T^{\prime}=\left(t_{2}, \ldots, t_{n}\right)$. Então

$$
\begin{aligned}
\left(\left.\xi\right|_{N}\right)_{T}(p) & =\left(\left.X\right|_{N}\right)_{t_{1}}\left(\left(\left.\psi\right|_{N}\right)_{T^{\prime}}(p)\right) \\
& =\left(\left.X\right|_{N}\right)_{t_{1}}\left(\psi_{T^{\prime}}(p)\right)=X_{t_{1}}\left(\psi_{T^{\prime}}(p)\right)=\xi_{T}(p) .
\end{aligned}
$$

Corolário 2.51. Na linguagem das aplicações $\rho$ que foram usadas para definir a topologia, a proposição acima diz: definidas

$$
\rho_{\xi, p}: \Omega_{\xi, p} \stackrel{a b .}{\subset} \mathbb{R}^{n} \longrightarrow M \quad \text { e } \quad \rho_{\left.\xi\right|_{N}, p}: \Omega_{\left.\xi\right|_{N}, p} \stackrel{a b .}{\subset} \mathbb{R}^{n} \longrightarrow N,
$$

tem-se que $\Omega_{\left.\xi\right|_{N}, p} \stackrel{a b .}{\subset} \Omega_{\xi, p}$ e $\rho_{\xi, p}(T)=\rho_{\left.\xi\right|_{N}, p}(T), \forall T \in \Omega_{\left.\xi\right|_{N}, p}$.

Exemplo 2.52. Seja $M=\mathbb{R}^{3}$. Então os planos horizontais da forma $z=k$ são subvariedades integrais maximais da distribuição $\Delta$ gerada por $\left\{\partial_{x}, \partial_{y}\right\}$.

Para mostrar que os planos horizontais são subvariedades integrais de $\Delta$, podemos escrever cada plano como uma imagem de imersão injetiva (de $\mathbb{R}^{2}$ em $\mathbb{R}^{3}$ ) e usar a proposição 1.38 .

Seja $S_{p}$ um desses planos horizontais. Mostremos que $S_{p}$ é maximal. Seja $N$ uma subvariedade integral conexa de $\Delta$ que passa por $p$. Mostremos primeiro que o conjunto de pontos de $N$ é um aberto em $S_{p}$. Os campos $\partial_{x}$ e $\partial_{y}$ são tangentes a $N$, então podemos restringi-los a $N$.

Vamos dar nome aos campos: $X=\partial_{x}$ e $Y=\partial_{y}$. Sejam $\xi=(X, Y)$ e $\eta=\left(\left.X\right|_{N},\left.Y\right|_{N}\right)=\left.\xi\right|_{N}$. Para cada $q \in N \cap S_{p}$, temos a aplicação suave $\rho_{\eta, q}: \Omega_{\eta, q} \stackrel{a b .}{\subset} \mathbb{R}^{2} \rightarrow N$, definida por

$$
\rho_{\eta, q}\left(t_{1}, t_{2}\right)=\eta_{\left(t_{1}, t_{2}\right)}(q)=\left(\left.X\right|_{N}\right)_{t_{1}}\left(\left(\left.Y\right|_{N}\right)_{t_{2}}(q)\right) .
$$

Pela proposição $2.50, \Omega_{\eta, q} \subset \Omega_{\xi, q} \mathrm{e}$

$$
\rho_{\eta, q}\left(t_{1}, t_{2}\right)=\rho_{\xi, q}\left(t_{1}, t_{2}\right)=\left(x(q)+t_{1}, y(q)+t_{2}\right), \quad \forall\left(t_{1}, t_{2}\right) \in \Omega_{\eta, q} .
$$

Como $\rho_{\eta, q}$ tem posto 2 e é injetiva, podemos argumentar pelo teorema da função inversa que $\rho_{\eta, q}$ tem imagem aberta em $N$. Como a imagem de $\rho_{\eta, q}$ é 
também um aberto em $S_{p}$ (pela fórmula acima), concluímos que todo ponto $q \in S_{p} \cap N$ possui uma vizinhança aberta em $N$ que também é um aberto de $S_{p}$.

Isto implica que $N \cap S_{p}$ é aberto em $S_{p}$. Mostremos que $N=N \cap S_{p}$. Dado $q \in N \backslash S_{p}$, $q$ está no plano horizontal $S_{q}=\left\{q^{\prime} \in \mathbb{R}^{3} \mid z\left(q^{\prime}\right)=z(q)\right\}$ e podemos usar um argumento análogo ao anterior para mostrar que $q$ tem uma vizinhança aberta em $N$ que está contida em $S_{q}$ - e, portanto, não em $S_{p}$. Assim, $N \backslash S_{p}$ também será aberto. Pela conexidade de $N, N \backslash S_{p}$ é vazio (porque $N \cap S_{p}$ não é).

Agora, mostremos que $N$ é uma subvariedade aberta de $S_{p}$. Seja $N_{2}$ a subvariedade aberta de $S_{p}$ (e, portanto, subvariedade imersa de $\mathbb{R}^{3}$ ) com o conjunto de pontos de $N$. Queremos mostrar que $N=N_{2}$. Seja $U$ um aberto de $N$. Então $U$ - visto como subvariedade aberta de $N$ - também é uma subvariedade integral de $\Delta$.

Se $U$ for conexo, $U$ será um subconjunto aberto de $S_{p}$, pelo argumento que foi feito anteriormente. Se $U$ não for conexo, cada componente conexa de $U$ é aberta em $S_{p}$ e, portanto, $U$ também o é.

Assim, todo conjunto aberto $U$ de $N$ é aberto em $S_{p}$ (e, portanto, em $N_{2}$ ). Isto implica que a topologia de $N$ é mais fina do que a topologia de $N_{2}$. Com o fato de que $\operatorname{dim} N=\operatorname{dim} N_{2}$, o corolário $1.49 \operatorname{nos} \operatorname{diz}$ que $N=N_{2}$.

\subsubsection{D-invariância}

Definição 2.53. Seja $D$ uma família de campos vetoriais na variedade $M$. Uma distribuição $\Delta$ é dita $D$-invariante quando, para toda $\phi \in G_{D}, \phi: \Omega \rightarrow$ $\Omega^{\prime}$, temos:

$$
\mathrm{d} \phi(\Delta(p)) \subset \Delta(\phi(p)), \forall p \in \Omega
$$

Definição 2.54. Dada uma família de campos vetoriais $D$ definida em todo lugar na variedade $M$, a distribuição $P_{D}$ é definida como a menor distribuição $D$-invariante contendo $\Delta_{D}$.

Definição 2.55. Seja $\phi: \Omega_{\phi} \rightarrow \Omega_{\phi}^{\prime}$ um difeomorfismo local em $M$. Dado um campo vetorial $X \in \mathfrak{X}\left(\Omega_{X}\right)$, com $\Omega_{X}$ aberto em $M$ interceptando $\Omega_{\phi}$, definimos o campo vetorial

$$
\mathrm{d} \phi(X) \in \mathfrak{X}\left(\phi\left(\Omega_{X} \cap \Omega_{\phi}\right)\right)
$$

da seguinte maneira: a cada ponto $\phi(p)$ associamos o vetor tangente $\mathrm{d} \phi\left(\left.X\right|_{p}\right)$. Ou seja, $\left.\mathrm{d} \phi(X)\right|_{q}=\mathrm{d} \phi\left(\left.X\right|_{\phi^{-1}(q)}\right)$. Isto será um campo vetorial suave, devido ao fato de $\phi^{-1}$ ser suave. 
Proposição 2.56 (Gerador de $P_{D}$ ). Dada uma família $D$ de campos vetoriais definida em todo lugar em $M$, seja

$$
A=\left\{\mathrm{d} \phi(X) \mid \phi \in G_{D}, X \in D\right\} .
$$

Então $P_{D}=\Delta_{A}$.

Demonstração. $\Delta_{A}$ contém $\Delta_{D}$ : pois $D \subset A$, pois, dado $X \in D$, a aplicação $X_{0}: \Omega_{X} \rightarrow \Omega_{X}$, que é a identidade no aberto $\Omega_{X}$ em que $X$ é definido, está em $G_{D}$. Então d $X_{0}(X)=X$ está em $A$.

$\Delta_{A}$ está contida em $P_{D}$ : Dado $\vec{v}_{p} \in \Delta_{A}(p), \vec{v}_{p}=\mathrm{d} \phi\left(\left.X\right|_{q}\right)$, para $X \in D$, $\phi \in G_{D}$ e $\phi(q)=p$. Como $\left.X\right|_{q}$ está contido em $P_{D}(q)$ e $P_{D}(q)$ é $D$-invariante, $\vec{v}_{p} \in P_{D}(p)$.

$\Delta_{A}$ é $D$-invariante: dados $\vec{v}_{p} \in \Delta_{A}(p)$ e $\psi \in G_{D}$, sabemos que $\vec{v}_{p}=$ $\mathrm{d} \phi\left(\left.X\right|_{q}\right)$, para $X \in D, \phi \in G_{D}$, e $\phi(q)=p$. Então queremos mostrar que $\mathrm{d} \psi\left(\vec{v}_{p}\right)$ está em $\Delta_{A}$. De fato:

$$
\mathrm{d} \psi\left(\mathrm{d} \phi\left(\left.X\right|_{q}\right)\right)=\mathrm{d}(\psi \phi)\left(\left.X\right|_{q}\right) \in \Delta_{A}
$$

pois $\phi g \in G_{D}$.

Exemplo 2.57. Em $\mathbb{R}^{3}$, a distribuição $\Delta_{D}$ gerada por

$$
D=\left\{\frac{\partial}{\partial x}, \frac{\partial}{\partial y}\right\}
$$

é $D$-invariante.

Seja $\vec{v}_{p} \in \Delta(p)$ um vetor tangente de $\Delta(p)$, ou seja,

$$
\vec{v}_{p}=\left.a \frac{\partial}{\partial x}\right|_{p}+\left.b \frac{\partial}{\partial y}\right|_{p}
$$

Mostremos que $\phi \in G_{D}$ implica $\mathrm{d} \phi\left(\vec{v}_{p}\right) \in \Delta(\phi(p))$. Podemos escrever (ver a observação 1.14) $\vec{v}_{p}=\alpha^{\prime}(0)$, onde $\alpha:(-\delta, \delta) \rightarrow \mathbb{R}^{3}$ é uma curva cuja imagem está contida no plano horizontal $S_{p}$ que passa por $p$. O diferencial $\mathrm{d} \phi\left(\vec{v}_{p}\right)$, então, pode ser calculado usando a curva $\alpha$ :

$$
\mathrm{d} \phi\left(\vec{v}_{p}\right)=\mathrm{d} \phi\left(\alpha^{\prime}(0)\right)=(\phi \circ \alpha)^{\prime}(0) .
$$

Como $S_{p}$ é uma $D$-órbita, $\phi\left(S_{p}\right) \subset S_{p}$ e portanto a imagem da curva $\phi \circ \alpha$ está contida em $S_{p}$. Temos que $z(\phi \circ \alpha)$ é constante, logo

$$
(\phi \circ \alpha)^{\prime}(0)=\left.d \frac{\partial}{\partial x}\right|_{\phi(p)}+\left.e \frac{\partial}{\partial y}\right|_{\phi(p)} \quad(\operatorname{com} d, e \in \mathbb{R}),
$$

que pertence a $\Delta(\phi(p))$. 
Exemplo 2.58. A distribuição gerada por $D=\left\{\partial_{x}, \partial_{y}+x \partial_{z}\right\}$ em $\mathbb{R}^{3}$ não é $D$-invariante.

De fato: sejam

$$
p=(1,0,0), \quad \vec{v}_{p}=\left.\frac{\partial}{\partial y}\right|_{p}+\left.\frac{\partial}{\partial z}\right|_{p} \in \Delta(p) .
$$

A translação $\phi=(x-1, y, z)$ pertence a $G_{D}$. Aplicando seu diferencial a $\vec{v}_{p}$, obtemos:

$$
\mathrm{d} \phi\left(\vec{v}_{p}\right)=\left.\frac{\partial}{\partial y}\right|_{0}+\left.\frac{\partial}{\partial z}\right|_{0} \notin \Delta(0)
$$

(note que $\phi(p)=0)$.

\subsubsection{Posto das $D$-órbitas}

Definição 2.59. Chamaremos $\operatorname{dim} P_{D}(p)$ de posto da D-órbita que passa pelo ponto $p$.

Observação 2.60. É preciso mostrar que esta definição não depende da escolha do ponto $p$.

Proposição 2.61. Seja D uma familia de campos vetoriais definida em todo lugar na variedade $M$. Se p e q são dois pontos na mesma D-órbita, então $\operatorname{dim} P_{D}(p)=\operatorname{dim} P_{D}(q)$.

Demonstração. Seja $\phi \in G_{D}$ tal que $\phi(p)=q$. Como $\phi$ é um difeomorfismo local, $\left.\mathrm{d} \phi\right|_{p}: \mathrm{T}_{p} M \rightarrow \mathrm{T}_{q} M$ é um isomorfismo. $\phi\left(P_{D}(p)\right) \subset P_{D}(q)$, pela $D$-invariância de $P_{D}$. Assim, $\phi\left(P_{D}(p)\right)$ será um subespaço de dimensão $\operatorname{dim} P_{D}(p)$ de $P_{D}(q)$, o que implica $\operatorname{dim} P_{D}(q) \geq \operatorname{dim} P_{D}(p)$. Analogamente, $\operatorname{dim} P_{D}(q) \geq \operatorname{dim} P_{D}(p)$.

Exemplo 2.62. $D=\left\{\partial_{x}, \partial_{y}+x \partial_{z}\right\}$ em $\mathbb{R}^{3}$ Então $P_{D}(p)=\mathrm{T}_{p} \mathbb{R}^{3}, \quad \forall p \in \mathbb{R}^{3}$.

Como a única $D$-órbita é todo o $\mathbb{R}^{3}$, a dimensão de $P_{D}$ deve ser uma constante $k$. Mostremos que $P_{D}(0)$ tem dimensão 3.

Os vetores

$$
\left.\frac{\partial}{\partial x}\right|_{0} \quad \text { e }\left.\quad \frac{\partial}{\partial y}\right|_{0}
$$

estão em $P_{D}(0)$, por estarem em $\Delta_{D}$. O vetor

$$
\left.\frac{\partial}{\partial y}\right|_{0}+\left.\frac{\partial}{\partial z}\right|_{0}
$$


linearmente independente dos dois outros, também está em $P_{D}(0)$, pois é o resultado do diferencial da translação $(x-1, y, z)$ aplicado em

$$
\left.\frac{\partial}{\partial x}\right|_{(1,0,0)}+\left.\frac{\partial}{\partial z}\right|_{(1,0,0)} \in \Delta_{D}(1,0,0) .
$$

Assim, $P_{D}(p)$ tem dimensão 3 em cada $p \in \mathbb{R}^{3}$, ou seja, é igual a todo o espaço tangente.

\subsubsection{Involutividade}

Definição 2.63. Uma distribuição $\Delta$ é involutiva se, dados $X, Y \in D_{\Delta}$, $X \in \mathfrak{X}\left(\Omega_{1}\right), Y \in \mathfrak{X}\left(\Omega_{2}\right), \Omega_{1}, \Omega_{2} \stackrel{a b}{\subset} M$, o colchete de Lie ou comutador $[X, Y]$ (definido em $\Omega_{1} \cap \Omega_{2}$ ) pertence a $D_{\Delta}$.

Proposição 2.64. Uma distribuição que possui a propriedade das subvariedades integrais é involutiva.

Demonstração. Sejam dois campos $X, Y \in \Delta$ e seja $p \in M$. Existe uma subvariedade integral $N_{p} \subset M$ de $\Delta$ que passa por $p$. Os campos $X$ e $Y$ são tangentes a $N_{p}$ e podem ser restritos a ela. Assim, o colchete de Lie em $p$, $\left.[X, Y]\right|_{p}$, pode ser calculado na subvariedade $N_{p}$ :

$$
\left.[X, Y]\right|_{p}=\left.\left[\left.X\right|_{N_{p}},\left.Y\right|_{N_{p}}\right]\right|_{p} \in \mathrm{T} N_{p} \subset \Delta(p)
$$

Exemplo 2.65. Seja $M=\mathbb{R}^{3}, D=\left\{\partial_{x}, \partial_{y}\right\}, \Delta=\Delta_{D}$. $\Delta$ é involutiva.

$$
D_{\Delta}=\left\{\left.a \frac{\partial}{\partial x}\right|_{\Omega}+\left.b \frac{\partial}{\partial y}\right|_{\Omega} \mid a, b \in \mathcal{C}^{\infty}(\Omega), \Omega \text { aberto em } \mathbb{R}^{3}\right\} .
$$

Dados

$$
X_{1}=\left.a_{1} \frac{\partial}{\partial x}\right|_{\Omega_{1}}+\left.b_{1} \frac{\partial}{\partial y}\right|_{\Omega_{1}} \text { e } X_{2}=\left.a_{2} \frac{\partial}{\partial x}\right|_{\Omega_{2}}+\left.b_{2} \frac{\partial}{\partial y}\right|_{\Omega_{2}},
$$

o campo $[X, Y]$ é definido em $\Omega_{1} \cap \Omega_{2}$. Como $X$ e $Y$ não envolvem a derivada parcial na coordenada $z$, seu colchete de Lie $[X, Y]$ também não a envolverá e, portanto, $[X, Y] \in D_{\Delta}$.

Observação 2.66. Mais geralmente, se $\Delta$ é uma distribuição tal que todo ponto de $M$ tem uma vizinhança aberta na qual a restrição de $\Delta$ é gerada pelas primeiras $k$ derivadas parciais $\left(\partial_{x_{1}}, \ldots, \partial_{x_{k}}\right)$ de algum sistema de coordenadas locais $\vec{x}=\left(x_{1}, \ldots, x_{n}\right)$, (onde $n$ é a dimensão de $M$ e $k \leq n$ é constante nas componentes conexas de $M) \Delta$ é involutiva. 
Exemplo 2.67. Novamente em $\mathbb{R}^{3}$, seja $D=\left\{\partial_{x}, \partial_{y}+x \partial_{z}\right\}$. Então $\Delta:=\Delta_{D}$ não é involutiva. De fato, dando os nomes $X=\partial_{x}$ e $Y=\partial_{y}+x \partial_{z}$,

$$
[X, Y]=X Y-Y X=\left(\frac{\partial^{2}}{\partial x \partial y}+\frac{\partial}{\partial z}+x \frac{\partial^{2}}{\partial x \partial z}\right)-\left(\frac{\partial^{2}}{\partial y \partial x}+x \frac{\partial^{2}}{\partial z \partial x}\right)=\frac{\partial}{\partial z}
$$

O campo $\partial_{z}$ não pertence à distribuição $\Delta$, pois $\Delta(0)$ é o espaço gerado por $\left.\partial_{x}\right|_{0}$ e $\left.\partial_{y}\right|_{0}$.

Exemplo 2.68. Seja $M=\mathbb{R}^{2}$ com as coordenadas usuais $(x, y)=\mathrm{Id}$. Seja $\phi \in \mathcal{C}^{\infty}(\mathbb{R})$ dada por

$$
\phi(t)= \begin{cases}\mathrm{e}^{-1 / t}, & \text { se } t>0 \text { e } \\ 0, & \text { se } t \leq 0 .\end{cases}
$$

Seja $\Delta$ a distribuição gerada por

$$
D=\left\{\frac{\partial}{\partial x}, \phi(x) \frac{\partial}{\partial y}\right\} .
$$

$\Delta$ não é $D$-invariante mas é involutiva.

Para mostrar que $\Delta$ não é $D$-invariante, basta reparar que o vetor tangente

$$
\left.\frac{\partial}{\partial y}\right|_{(1,0)} \in \Delta(1,0)
$$

é levado em

$$
\left.\frac{\partial}{\partial y}\right|_{(-1,0)} \notin \Delta(-1,0)
$$

pelo diferencial da translação $(x-2, y)$, que está em $G_{D}$, gerada pelo fluxo de $\partial_{x}$.

$\Delta$ é involutiva: Dados dois campos $X, Y \in \Delta$, suponha que eles são definidos em um aberto $\Omega \subset \mathbb{R}^{2}$, com $p \in \Omega$. Devemos mostrar que $\left.[X, Y]\right|_{p} \in$ $\Delta(p)$. Se $x(p)>0$, então $\Delta(p)=\mathrm{T}_{p} \mathbb{R}^{2}$ e portanto $\left.[X, Y]\right|_{p} \in \Delta(p)$.

Se $x(p)<0$, podemos supor que o $\Omega \subset\{x<0\}$. Então $X$ e $Y$ não envolverão a derivação $\partial_{y}$. Logo, $[X, Y]$ não envolverá a derivação $\partial_{y}$. Assim, teremos $\left.[X, Y]\right|_{p} \in \Delta(p)$.

Se $x(p)=0$ : queremos mostrar que $\left.[X, Y]\right|_{p}$ é um múltiplo de $\left.\frac{\partial}{\partial x}\right|_{p}$. Vamos escrever

$$
\begin{aligned}
& X=a \frac{\partial}{\partial x}+b \frac{\partial}{\partial y} \mathrm{e} \\
& Y=c \frac{\partial}{\partial x}+d \frac{\partial}{\partial y}
\end{aligned}
$$


Com $b(q)=0$ e $d(q)=0$ sempre que $x(q) \leq 0$. Pela suavidade de $b$ e $d$, as derivadas

$$
\frac{\partial b}{\partial x}(p), \frac{\partial d}{\partial x}(p), \frac{\partial b}{\partial y}(p), \frac{\partial d}{\partial y}(p)
$$

serão nulas. Para calcular $[X, Y]$, usamos a fórmula:

$$
[X, Y]=(X Y-Y X)(x) \frac{\partial}{\partial x}+(X Y-Y X)(y) \frac{\partial}{\partial y} .
$$

$X Y(y)=X d=0$ em $p$, e $Y X(y)=Y b=0$ em $p$. Assim, $\left.[X, Y]\right|_{p}$ não envolverá $\left.\partial_{y}\right|_{p}$, que é o que queríamos demonstrar.

\subsection{Teoremas principais}

\subsubsection{Que as $D$-órbitas são subvariedades}

\section{Direções de movimento em uma órbita}

Seja $D$ uma família definida em todo lugar de campos vetoriais na variedade $M$. Dados $\xi=\left(X^{1}, \ldots, X^{n}\right) \in D^{n}, p \in M$ e $T \in \Omega_{\xi, p} \stackrel{a b}{\subset} \mathbb{R}^{n}$, sabemos que a aplicação $T^{\prime} \mapsto \rho_{\xi, p}\left(T^{\prime}\right)=\xi_{T^{\prime}}(p)$, é suave em $\Omega_{\xi, p}$.

Definição 2.69. $V(\xi, p, T)$ é a imagem do diferencial $\left.\mathrm{d}\left(\rho_{\xi, p}\right)\right|_{T}$, isto é,

$$
V(\xi, p, T)=\mathrm{d}\left(\rho_{\xi, p}\right)\left(\mathrm{T}_{T} \Omega_{\xi, p}\right) .
$$

Lema 2.70 (Lema (5.1) de Sussmann [10]).

$$
V(\xi, p, T) \subset P_{D}\left(\xi_{T}(p)\right) .
$$

Demonstração. Seja $\left(x_{1}, \ldots, x_{n}\right)=\left.\operatorname{Id}\right|_{\Omega \xi, p}$, a carta global usual de $\Omega_{\xi, p}$. $V(\xi, p, T)$ é gerado pelos vetores tangentes

$$
\left.\frac{\partial \rho_{\xi, p}}{\partial x_{1}}\right|_{T}, \ldots,\left.\frac{\partial \rho_{\xi, p}}{\partial x_{n}}\right|_{T}=\mathrm{d} \rho_{\xi, p}\left(\left.\frac{\partial}{\partial x_{1}}\right|_{T}\right), \ldots, \mathrm{d} \rho_{\xi, p}\left(\left.\frac{\partial}{\partial x_{n}}\right|_{T}\right) .
$$

Queremos, então, mostrar que estes diferenciais estão em $P_{D}$. Partimos de

$$
\rho_{\xi_{p}}=X_{x_{1}}^{1}\left(X_{x_{2}}^{2}\left(\ldots\left(X_{x_{n}}^{n}(p)\right) \ldots\right)\right) \text {. }
$$

A expressão acima, ao se variar $x_{1}$, e mantendo $x_{2}=t_{2}, \ldots, x_{n}=t_{n}$ constantes, será uma curva integral do campo $X_{1}$ - isto é,

$$
\alpha(t):=\rho_{\xi, p}\left(t, t_{2}, \ldots, t_{n}\right)
$$


(que pode ser definida para $t \in\left(t_{0}-\delta, t_{0}+\delta\right)$, para algum $\delta>0$ ) é uma curva integral de $X_{1}$.

Pela proposição 1.21 (cálculo do diferencial por caminhos) e usando que $\alpha$ é curva integral de $X^{1}$, obtemos:

$$
\left.\frac{\partial \rho_{\xi, p}}{\partial x_{1}}\right|_{T}=\mathrm{d} \rho_{\xi, p}\left(\left.\frac{\partial}{\partial x_{1}}\right|_{T}\right)=\alpha^{\prime}\left(t_{1}\right)=\left.X^{1}\right|_{X_{t_{1}}^{1}\left(X_{t_{2}}^{2}\left(\ldots\left(X_{t_{n}}^{n}(p)\right) \ldots\right)\right)} .
$$

Para calcular as outras derivadas parciais, usa-se a regra da cadeia e repetimos o argumento anterior:

$$
\begin{aligned}
\frac{\partial \rho_{\xi, p}}{\left.\partial x_{2}\right|_{T}} & =\mathrm{d} X_{t_{1}}^{1}\left(\mathrm{~d}\left(X_{x_{2}}^{2}\left(\ldots\left(X_{x_{n}}^{n}(p)\right) \ldots\right)\right)\left(\left.\frac{\partial}{\partial x_{2}}\right|_{\left(t_{1}, t_{2}, \ldots, t_{n}\right)}\right)\right) \\
& =\mathrm{d} X_{t_{1}}^{1}\left(\frac{\partial}{\partial x_{2}}\left(X_{x_{2}}^{2}\left(\ldots\left(X_{x_{n}}^{n}(p)\right) \ldots\right)\right)\left(t_{1}, t_{2}, \ldots, t_{n}\right)\right) \\
& =\mathrm{d} X_{t_{1}}^{1}\left(\frac{\partial}{\partial y_{1}}\left(X_{y_{1}}^{2}\left(\ldots\left(X_{y_{n-1}}^{n}(p)\right) \ldots\right)\right)\left(t_{2}, \ldots, t_{n}\right)\right) \\
& =\mathrm{d} X_{t_{1}}^{1}\left(\left.X^{2}\right|_{X_{t_{2}}^{2}\left(\ldots\left(X_{t_{n}}^{n}(p)\right) \ldots\right)}\right), \\
& \vdots \\
\frac{\left.\partial \rho_{\xi, p}\right|_{\partial x_{n}}}{2} & =\mathrm{d} X_{t_{1}}^{1}\left(\ldots\left(\mathrm{d} X_{t_{n-1}}^{n-1}\left(\left.X^{n}\right|_{X_{t_{n}}^{n}(p)}\right)\right) \ldots\right) .
\end{aligned}
$$

Estes vetores são todos pertencentes a $P_{D}$, por serem obtidos pela aplicação repetida dos diferenciais de elementos de $G_{D}-X_{t_{1}}^{1}, \ldots, X_{t_{n}}^{n}$ - a vetores dos campos $X^{1}, \ldots, X^{n}$, que são pertencentes à distribuição $D$-invariante $P_{D}$.

Lema 2.71 (Lema (5.2) de Sussmann [10]). Para todo $q \in M$, podemos escolher $\xi, p, T$ de forma que $q=\xi_{T}(p) e$

$$
V(\xi, p, T)=P_{D}(q)
$$

Demonstração. Pela proposição 2.56, o conjunto

$$
A=\left\{\mathrm{d} \phi\left(\left.X\right|_{p}\right) \mid \phi \in G_{D}, X \in D, p \in \phi^{-1}(\{q\}) \cap \operatorname{Dom} X\right\}
$$

de vetores tangentes em $q$ é um gerador de $P_{D}(q)$.

Seja $\left(\vec{u}_{1}, \ldots, \vec{u}_{k}\right)$ uma base de $P_{D}(q)$ com $\vec{u}_{1}, \ldots, \vec{u}_{k} \in A$.

Então, para todo $j \in\{1, \ldots, k\}$, podemos escrever

$$
\vec{u}_{j}=\mathrm{d}\left(\xi_{j_{T_{j}}}\right)\left(\left.X^{j}\right|_{p_{j}}\right)
$$


onde $\xi_{j} \in D^{n_{j}}, T_{j} \in \mathbb{R}^{n_{j}}, X^{j} \in D$ e $p_{j} \in \operatorname{Dom} X_{j}$ é tal que $\xi_{j_{j}}\left(p_{j}\right)=q$.

Assim, temos $\vec{u}_{j} \in V\left(\xi_{j}, p_{j}, T_{j}\right)$.

Faça $\xi=\xi_{k} \widehat{\xi_{k}} \cdots \xi_{2} \widehat{\xi}_{2} \xi_{1}, p=p_{1}$ e $T=T_{k}\left(-\widehat{T_{k}}\right) \cdots T_{2}\left(-\widehat{T}_{2}\right) T_{1}$. Aqui, estamos usando a notação que foi introduzida na demonstração da proposição 2.28 .

Afirmação: $V(\xi, T, p)=P_{D}(q)$. Para provar isto, basta mostrar que a imagem do diferencial da função

$$
\rho_{\xi, p}: \Omega_{\xi, p} \stackrel{a b .}{\subset} \mathbb{R}^{n_{1}+2\left(\sum_{j=2}^{k} n_{j}\right)} \longrightarrow M
$$

em $T$ contém os espaços $V\left(\xi_{j}, p_{j}, T_{j}\right), \quad j=1, \ldots, k$.

$$
\rho_{\xi, p}(T)=\xi_{k T_{k}}\left(\xi_{k T_{k}}\right)^{-1} \cdots \xi_{2 T_{2}}\left(\xi_{2 T_{2}}\right)^{-1} \xi_{1 T_{1}}\left(p_{1}\right)=\xi_{1 T_{1}}\left(p_{1}\right)=q .
$$

Vamos nomear as coordenadas de $\Omega_{\xi, p}$ :

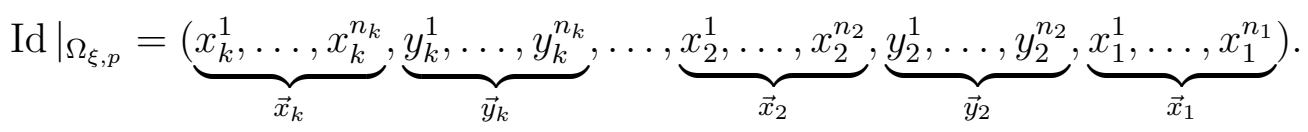

Assim, temos:

$$
\begin{gathered}
\rho_{\xi, p}=\left(\xi_{k}\right)_{\vec{x}_{k}}\left(\widehat{\xi_{k}}\right)_{\vec{y}_{k}} \cdots\left(\xi_{2}\right)_{\vec{x}_{2}}\left(\widehat{\xi_{2}}\right)_{\vec{y}_{2}}\left(\xi_{1}\right)_{\vec{x}_{1}}, \\
\rho_{\xi, p}\left(T_{k},-\widehat{T_{k}}, \cdots, T_{2},-\widehat{T}_{2}, \vec{x}_{1}\right)=\xi_{\vec{x}_{1}}\left(p_{1}\right)=\rho_{\xi_{1}, q_{1}}\left(\vec{x}_{1}\right), \\
\rho_{\xi, p}\left(T_{k},-\widehat{T_{k}}, \cdots, \vec{x}_{2},-\widehat{T}_{2}, T_{1}\right)=\xi_{2 \vec{x}_{2}}\left(p_{2}\right)=\rho_{\xi_{2}, q_{2}}\left(\vec{x}_{2}\right), \\
\vdots \\
\rho_{\xi, p}\left(\vec{x}_{k},-\widehat{T_{k}}, \cdots, T_{2},-\widehat{T}_{2}, T_{1}\right)=\xi_{k_{\vec{x}_{k}}}\left(p_{k}\right)=\rho_{\xi_{k}, q_{k}}\left(\vec{x}_{k}\right) .
\end{gathered}
$$

Isto implica que o espaço gerado pelas derivadas parciais

$$
\left.\frac{\partial \rho_{\xi, p}}{\partial x_{j}^{1}}\right|_{T}, \ldots,\left.\frac{\partial \rho_{\xi, p}}{\partial x_{j}^{n_{j}}}\right|_{T}
$$

é $V\left(\xi_{j}, p_{j}, T_{j}\right)$, para todo $j \in\{1, \ldots, k\}$.

\section{Subvariedades integrais de $P_{D}$}

Lema 2.72 (Lema 5.3 de Sussmann [10]). Seja D uma família definida em todo lugar de campos vetoriais na variedade $M$. Seja $S$ uma D-órbita de $p$, dotada da topologia da D-órbita. Então toda subvariedade integral conexa de $P_{D}$ que intercepta $S$ é (vista como conjunto) um subconjunto aberto de $S$. 
Demonstração. Seja $N$ uma subvariedade integral conexa de $P_{D}$ que intercepta a $D$-órbita $S$. Mostremos primeiro que $N \subset S$.

Afirmação: dado $p \in N \cap S$ existe uma vizinhança aberta de $p$ em $N$ que está contida em $S$. De fato, pela proposição 2.56 , sabemos que a família

$$
A=\left\{\mathrm{d} \phi(X) \mid \phi \in G_{D}, X \in D\right\}
$$

de campos vetoriais gera $P_{D}$. Assim, podemos escolher campos $X^{1}, \ldots, X^{k} \in$ $D$ e difeomorfismos locais $\phi_{1}, \ldots, \phi_{k} \in G_{D}$ tais que

$$
\left.\mathrm{d} \phi_{1}\left(X^{1}\right)\right|_{p}, \ldots,\left.\mathrm{d} \phi_{k}\left(X^{k}\right)\right|_{p}
$$

são uma base para $P_{D}(p)=\mathrm{T}_{p} N$, onde $k=\operatorname{dim} N=\operatorname{dim} P_{D}(p)$.

Sejam $Y^{1}=\mathrm{d} \phi_{1}\left(X^{1}\right), \ldots, Y^{k}=\mathrm{d} \phi_{k}\left(X^{k}\right)$. Estes campos são tangentes a $N$, pois $N$ é subvariedade integral de $P_{D}$.

Sejam $\xi=\left(Y^{1}, \ldots, Y^{k}\right)$ e $\left.\xi\right|_{N}=\left(\left.Y^{1}\right|_{N}, \ldots,\left.Y^{k}\right|_{N}\right)$ e considere as aplicações

$$
\rho_{\xi, p}: \Omega_{\xi, p} \stackrel{a b .}{\subset} \mathbb{R}^{k} \longrightarrow M \quad \text { e } \quad \rho_{\left.\xi\right|_{N}, p}: \Omega_{\left.\xi\right|_{n}, p} \stackrel{a b .}{\subset} \mathbb{R}^{k} \longrightarrow N .
$$

Pelo corolário da proposição 2.50, $\Omega_{\left.\xi\right|_{N}, p} \stackrel{a b .}{\subset} \Omega_{\xi, p}$ e $\rho_{\xi, p}(T)=\rho_{\left.\xi\right|_{N}, p}(T)$, $\forall T \in \Omega_{\left.\xi\right|_{n}, p}$.

Como a imagem de $\rho_{\xi, p}$ está contida na $D$-órbita $S$ de $p$, então a imagem de $\rho_{\left.\xi\right|_{N}, p}$ também está. Como $\rho_{\left.\xi\right|_{N}, p}$ tem posto $k$ na origem, o teorema da função inversa nos diz que a sua imagem contém um aberto, em $N$, contendo $p$. Assim, $p$ tem uma vizinhança aberta em $N$ que está contida em $S$.

Variando $p$, isso implica que $N \cap S$ é aberto em $N$. Mostremos que $N \backslash S$ também é aberto em $N$. Dado $q \in N \backslash S$, temos que $q$ está na $D$-órbita $S_{q}$, disjunta de $S$. Usando o argumento anterior, podemos mostrar que $q$ tem uma vizinhança aberta (em $N$ ) que está contida em $S_{q}$, e portanto que não intercepta $S$. Variando $q$, temos que $N \backslash S$ é aberto em $N$.

Pela conexidade de $N$, e como estamos supondo, por hipótese, que $N \cap S$ não é vazio, $N \backslash S$ será vazio e portanto $N \subset S$.

Agora, devemos mostrar que o conjunto de pontos de $N$ é aberto na topologia da órbita $S$. Isto é, para quaisquer $m \in \mathbb{N}, \eta \in D^{m}$, queremos que o conjunto

$$
\rho_{\eta, p}^{-1}(N)=\left\{T^{\prime} \in \Omega_{\eta, p} \mid \rho_{\eta, p}\left(T^{\prime}\right) \in N\right\}
$$

seja aberto em $\mathbb{R}^{m}$. Seja $T \in \rho_{\eta, p}^{-1}(N)$. Mostremos que $T$ tem uma vizinhança aberta em $\rho_{\eta, p}^{-1}(N)$.

Faremos uma indução finita para provar que a seguinte proposição é verdadeira para todo $m \in \mathbb{N}$ : se $N$ é uma subvariedade integral de $P_{D}$ contida na $D$-órbita $S$, então $\rho_{\eta, p}^{-1}(N)$ é aberto para toda $\eta \in D^{m}$. 
Em primeiro lugar, podemos supor sem perda de generalidade que $N$ é uma subvariedade mergulhada, pois (observação 1.53) sempre podemos decompor $N$ em abertos que são subvariedades mergulhadas de $M$.

Se $m=1$ : Então $\rho_{\eta, p}$ é a curva integral maximal $\alpha:(a, b) \rightarrow M$ de um campo $Z \in D$ com $T=t \in(a, b)$ e $\alpha(t)=p$. Tendo $\alpha(t) \in N$, queremos achar $\delta>0$ tal que $\alpha((t-\delta, t+\delta)) \subset N$.

Seja $\alpha^{*}:(c, d) \rightarrow N$ a curva integral maximal de $\left.Z\right|_{N}$ tal que $\alpha^{*}(t)=p$. Como a inclusão de $\alpha^{*}$ é curva integral de $Z$ e vale $p$ no parâmetro $t$, então $\alpha^{*}$ e $\alpha$ coincidem em $(c, d)$. Assim, fazendo $\delta=\min \{t-c, d-t\}$, temos $\rho_{\eta, p}((t-\delta, t+\delta)) \subset N$.

Se $m>1$ : Então $\eta=(Z) \sigma$, com $Z \in D, \sigma \in D^{m-1}$. Escreva $T=$ $\left(t_{1}, \ldots, t_{m}\right)$. Então

$$
\rho_{\eta, p}\left(t_{1}, \ldots, t_{m}\right)=Z_{t_{1}}\left(\rho_{\sigma, p}\left(t_{2}, \ldots, t_{m}\right)\right) \in N
$$

Vamos usar o fato (consequência da proposição 1.58 e de que difeomorfismos são homeomorfismos) de que a imagem de uma subvariedade mergulhada por um difeomorfismo é outra subvariedade mergulhada. Com isso, $Z_{t_{1}}^{-1}(N)$ será uma subvariedade integral mergulhada de $P_{D}$.

Seja $V$ uma vizinhança de $\rho_{\sigma, p}\left(t_{2}, \ldots, t_{m}\right)$ em $Z_{t_{1}}^{-1}(N)$ tal que, para cada $q \in V$, para as curvas integrais

$$
\alpha_{q}(h)=\left(\left.Z\right|_{Z_{t_{1}}^{-1}(N)}\right)_{h}(q)
$$

são definidas em $Z_{t_{1}}^{-1}(N)$ no intervalo $h \in(-\delta, \delta)$, com algum $\delta>0$ independente de $q$. A existência de tal $\delta$ é consequência da proposição 2.3 (existência e unicidade local de curva integral). Temos, como consequência da propriedade de grupo (proposição 2.13) que, dados $q \in V, h \in(-\delta, \delta)$,

$$
Z_{t_{1}}\left(\alpha_{q}(h)\right)=Z_{t_{1}}\left(Z_{h}(q)\right)=Z_{t_{1}+h}(q) \in N
$$

Como $Z_{t_{1}}^{-1}(N)$ é subvariedade mergulhada, $V=Z_{t_{1}}^{-1}(N) \cap V_{M}$, para um aberto $V_{M}$ de $M$. Pela continuidade de $\rho_{\sigma, p}$, existe um aberto $U^{\prime} \subset \mathbb{R}^{m-1}$ tal que $\rho_{\sigma, p}\left(U^{\prime}\right) \subset V_{M}$. Como $\rho_{\sigma, p}^{-1}\left(Z_{t_{1}}^{-1}(N)\right)$ é um aberto $U^{\prime \prime} \subset \mathbb{R}^{m-1}$ pela hipótese de indução, a intersecção $\Omega=U^{\prime} \cap U^{\prime \prime}$ será um aberto em $\mathbb{R}^{m-1} \mathrm{e}$, assim, $\rho_{\sigma, p}(\Omega) \subset Z_{t_{1}}^{-1}(N) \cap V$. Portanto,

$$
\rho_{\sigma, p}(\Omega) \subset V \quad \therefore \rho_{\eta, p}(\underbrace{\left(t_{1}-\delta, t_{1}+\delta\right) \times \Omega}_{\text {aberto em } \mathbb{R}^{m}}) \subset N .
$$

Isto mostra que o conjunto de pontos de $N$ é aberto em $S$. 
Teorema 2.73 (Teorema 4.1 de Sussmann [10]). Seja $M$ uma variedade e seja $D$ uma familia definida em todo lugar de campos vetoriais reais suaves.

(a) Cada D-órbita, com a sua topologia de órbita, admite uma única estrutura diferenciável que a torna uma subvariedade imersa.

(b) As D-órbitas, com a estrutura diferenciável acima, são subvariedades integrais maximais de $P_{D}$.

(c) $P_{D}$ tem a propriedade das variedades integrais maximais.

(d) $P_{D}$ é involutiva.

Demonstração. Seja $S$ uma $D$-órbita. Mostremos que $S$, com a topologia de $D$-órbita, é uma subvariedade imersa. Seja $p \in S$. Pelo lema 2.71, existem $n \in \mathbb{N}, \xi \in D^{n}, T \in \mathbb{R}^{n}$ e $q \in S$ tais que $V(\xi, q, T)=P_{D}(p)$.

Seja $k=\operatorname{dim} P_{D}(p)$. Considere a aplicação $\rho_{\xi, q}: \Omega_{\xi, q} \rightarrow M$. O posto de $\rho_{\xi, q}$ é igual a $k$ em $T$, pelo que foi dito acima, e nunca excede $k$, pelo lema 2.70 .

Como o posto de uma aplicação suave nunca decresce subitamente, existe uma vizinhança $\mathcal{O}$ de $T$ na qual o posto de $\rho_{\xi, q}$ é igual a $k$.

Considere $\mathcal{O}$ como variedade. Vamos usar o teorema do posto em $\left.\rho_{\xi, q}\right|_{\mathcal{O}}$ em torno do ponto $T$ : existem cartas locais

$$
\begin{array}{cc}
\vec{x}: U \stackrel{a b .}{\subset} \mathcal{O} \longrightarrow V \stackrel{a b .}{\subset} \mathbb{R}^{n}, & T \in U \quad \mathrm{e} \\
\vec{y}: U^{\prime} \stackrel{a b .}{\subset} M \longrightarrow V^{\prime} \stackrel{a b .}{\subset} \mathbb{R}^{m}, & p \in U^{\prime}
\end{array}
$$

tais que

$$
\begin{aligned}
\rho_{\xi, q}(U) \subset U^{\prime}, & =W \times(-\delta, \delta)^{n-k} \\
V^{\prime} & =W \times(-\delta, \delta)^{m-k},
\end{aligned}
$$

sendo que $W \stackrel{a b .}{\subset} \mathbb{R}^{k}$ e nas quais se escreve:

$$
\vec{y} \circ\left(\left.\rho_{\xi, q}\right|_{U \rightarrow U^{\prime}}\right)=\left.(x_{1}, \ldots, x_{k}, \underbrace{0, \ldots, 0}_{m-k})\right|_{U \rightarrow V^{\prime}} .
$$

O conjunto $N=\vec{x}^{-1}\left(W \times\{0\}^{n-k}\right)$ se torna uma subvariedade imersa de $U$ de dimensão $k$, quando damos a ele a estrutura diferenciável induzida pela carta global $\left.\left(x_{1}, \ldots, x_{k}\right)\right|_{N}$. O fibrado tangente de $N$ será o espaço gerado por

$$
\left.\frac{\partial}{\partial x_{1}}\right|_{N}, \ldots,\left.\frac{\partial}{\partial x_{k}}\right|_{N}
$$


Pela equação (2.74), vemos que $\left.\rho_{\xi, q}\right|_{N}$ será uma imersão injetiva, com diferencial igual ao de $\rho_{\xi, q}$ nos pontos de $U$, que tem posto $k$ em cada ponto e, pelo lema 2.70, está contido em $P_{D}$. Portanto, $\mathrm{d}\left(\left.\rho_{\xi, q}\right|_{N}\right)\left(T^{\prime}\right)=P_{D}\left(T^{\prime}\right), \quad \forall T^{\prime} \in U$.

$\left.\rho_{\xi, q}\right|_{N}$ é uma imersão injetiva e portanto a sua imagem, $N^{\prime}=\left.\rho_{\xi, q}\right|_{N}(N)$, será uma subvariedade integral de $P_{D}$ que passa pelo ponto $p$ (pela proposição 1.38).

Mostremos que a topologia de $N^{\prime}$ é a mesma que a induzida por $S$ em $N^{\prime}$. Dado um aberto conexo $\Omega \subset N^{\prime}$, visto como subvariedade aberta, $\Omega$ será uma subvariedade integral conexa de $P_{D}$. Assim, pelo lema 2.70, $\Omega$ é um aberto de $S$. Como todo aberto de $N^{\prime}$ é uma união de abertos conexos (variedades são localmente conexas), temos que todo aberto de $N^{\prime}$ é aberto na topologia de $S$. Assim, o conjunto de pontos de $N^{\prime}$ é aberto em $S$ e a topologia de $N^{\prime}$ é mais fina que a topologia de $\left.S\right|_{N^{\prime}}$.

Por outro lado, $\left.\rho_{\xi, q}\right|_{N \rightarrow N^{\prime}}$ é um homeomorfismo entre $N$ e $N^{\prime}$, pois a topologia de $N^{\prime}$ é a co-induzida por $\left.\rho_{\xi, q}\right|_{N}$ (proposição 1.38). Dado $\Omega^{\prime}$ um aberto de $\left.S\right|_{N^{\prime}}$, ou seja, um aberto em $S$ que está contido em $N^{\prime}$, queremos mostrar que $\Omega^{\prime}$ é aberto em $N^{\prime}$.

Como $\Omega^{\prime}$ é aberto em $S, \rho_{\xi, q}^{-1}\left(\Omega^{\prime}\right)$ é aberto em $\Omega_{\xi, q}$.

$$
\Omega^{\prime \prime}:=\left(\left.\rho_{\xi, q}\right|_{U}\right)^{-1}\left(\Omega^{\prime}\right)=U \cap \rho_{\xi, q}^{-1}\left(\Omega^{\prime}\right)
$$

também é aberto. Por (2.74),

$$
\vec{y}\left(\Omega^{\prime \prime}\right)=\mathcal{A} \times(-\delta, \delta)^{n-k},
$$

onde $\mathcal{A} \subset \mathbb{R}^{k}$. Como este conjunto é aberto, concluímos que $\mathcal{A}$ é aberto. Seja $\Omega^{(3)}=\left(\left.\rho_{\xi, q}\right|_{N}\right)^{-1}\left(\Omega^{\prime}\right)$. Novamente por (2.74), obtemos

$$
\left.\left(x_{1}, \ldots, x_{k}\right)\right|_{N}\left(\Omega^{(3)}\right)=\mathcal{A} .
$$

Como $\left.\left(x_{1}, \ldots, x_{k}\right)\right|_{N}$ é carta local de $N$, temos que $\Omega^{(3)}$ é aberto em $N$. Como $\left(\left.\rho_{\xi, q}\right|_{N \rightarrow N^{\prime}}\right)^{-1}$ é homeomorfismo, concluímos que $\Omega^{\prime}$ é aberto em $N^{\prime}$. Assim, temos que, para cada $p \in S$, existe uma subvariedade integral de $P_{D}$ contendo o ponto $p$, contida em $S$, de dimensão $k$ e cuja topologia é a restrição da topologia de $S$. Variando $p$, obtemos uma cobertura de $S$ por subvariedades de mesma dimensão e de topologias compatíveis, que são conjuntos abertos em $S$. Temos as condições necessárias para para unir estas subvariedades em uma só (proposição 1.53), que terá o conjunto de pontos e a topologia de $S$. Está provado que toda órbita de Sussmann é uma subvariedade integral de $P_{D}$.

Maximalidade: Mostremos que $S$ é uma subvariedade integral maximal. Se $R$ é uma subvariedade integral conexa que intercepta $S$, pelo lema 2.72, 
o conjunto de pontos de $R$ é aberto em $S$. Como os abertos conexos de $R$ também são subvariedades integrais conexas de $P_{D}$, eles serão abertos em $S$. Como todo aberto de $R$ é uma união de abertos conexos de $R$, todo aberto de $R$ será aberto em $S$. $R$ tem a topologia mais fina do que a topologia de $\left.S\right|_{R}$ e ambos têm a mesma dimensão, portanto $R=\left.S\right|_{R}$, isto é, $R$ é subvariedade aberta de $S$.

Como todo ponto de $M$ tem uma $D$-órbita, $P_{D}$ possui a propriedade das variedades integrais maximais. Pela proposição $2.64, P_{D}$ é involutiva.

\subsubsection{Condições para que $\Delta_{D}=P_{D}$}

\section{Um lema sobre o colchete de Lie}

Lema 2.75. Sejam $X$ e $Y$ dois campos vetoriais em $M$ e $p \in M$. Seja $\epsilon>0$ tal que $X_{t}(p)$ está definido para todo $t \in(-\epsilon, \epsilon)$. Seja

$$
W(t)=\mathrm{d} X_{-t}\left(\left.Y\right|_{X_{t}(p)}\right) \in \mathrm{T}_{p} M
$$

um caminho (no espaço vetorial $\mathrm{T}_{p} M$ ). Então

$$
W^{\prime}(t)=\mathrm{d} X_{-t}\left(\left.[X, Y]\right|_{X_{t}(p)}\right) .
$$

Demonstração. A derivada em $\mathrm{T}_{p} M$ pode ser definida da seguinte forma: Seja $v_{1}, \ldots, v_{m}$ uma base de $\mathrm{T}_{p} M$. Então $W$ se decompõe como

$$
W(t)=\sum_{j=1}^{m} w_{j}(t) v_{j} .
$$

onde as funções $w_{j}$ são definidas unicamente. Definimos

$$
W^{\prime}(t)=\sum_{j=1}^{m} w_{j}^{\prime}(t) v_{j} .
$$

$W^{\prime}(t)$ é definida se e somente se as derivadas $w_{j}^{\prime}(t)$ existirem. É um simples exercício de álgebra linear mostrar que $W^{\prime}(t)$ não depende da escolha da base.

Mostremos, primeiro, que a derivada $W^{\prime}(t)$ existe. Como base de $\mathrm{T}_{p} M$, tome as derivadas parciais $\left.\partial_{x_{1}}\right|_{p}, \ldots,\left.\partial_{x_{m}}\right|_{p}$ de alguma carta local

$$
\vec{x}=\left(x_{1}, \ldots, x_{m}\right): U \subset M \rightarrow V \subset \mathbb{R}^{m}
$$


que tenha $p \in U$. Então

$$
W(t)=\left.\sum_{j=1}^{m} W(t)\left[\left.x_{j}\right|_{p}\right] \frac{\partial}{\partial x_{j}}\right|_{p} .
$$

Queremos mostrar que as funções $w_{j}(t):=W(t)\left[\left.x_{j}\right|_{p}\right]$ são suaves. Seja $\xi=(X, Y, X)$. Considere a aplicação

$$
\rho_{\xi, p}: \Omega_{\xi, p} \stackrel{a b .}{\subset} \mathbb{R}^{3} \longrightarrow M
$$

Seja $(a, b, c)=\left.\operatorname{Id}\right|_{\Omega_{\xi, p}}$ o sistema de coordenadas usuais em $\Omega_{\xi, p}$. A derivada parcial na coordenada $b$ é dada por:

$$
\frac{\partial}{\partial b} \rho_{\xi, p}=\frac{\partial}{\partial b} X_{a}\left(Y_{b}\left(X_{c}(p)\right)\right)=\mathrm{d} X_{a}\left(\left.Y\right|_{Y_{b} X_{c}(p)}\right) .
$$

Assim,

$$
W(t)=\mathrm{d} X_{-t}\left(\left.Y\right|_{X_{t}(p)}\right)=\frac{\partial}{\partial b} \rho_{\xi, p}(-t, 0, t)
$$

Portanto,

$$
\begin{array}{r}
w_{j}(t)=W(t)\left[\left.x_{j}\right|_{p}\right]=\frac{\partial}{\partial b} \rho_{\xi, p}(-t, 0, t)\left[\left.x_{j}\right|_{p}\right]=\left.\frac{\partial}{\partial b}\right|_{(-t, 0, t)}\left[\left.\left(\overline{x_{j}} \circ \rho_{\xi, p}\right)\right|_{(-t, 0, t)}\right] \\
=\partial_{2}\left(\overline{x_{j}} \circ \rho_{\xi, p}\right)(-t, 0, t),
\end{array}
$$

Onde $\overline{x_{j}} \in \mathcal{C}^{\infty}(M)$ é representante do germe $\left.x_{j}\right|_{p}$ - isto é, uma função que coincide com $x_{j}$ em um aberto contendo $p$. Como $\rho_{\xi, p}$ é uma função suave, $\partial_{2}\left(\overline{x_{j}} \circ \rho_{\xi, p}\right)$ é infinitamente diferenciável, e assim $w_{j}(t)=\partial_{2}\left(\overline{x_{j}} \circ \rho_{\xi, p}\right)(-t, 0, t)$. também o será.

Calculemos então $w_{j}^{\prime}(t)$ :

$$
\begin{aligned}
w_{j}^{\prime}(t) & =\frac{\mathrm{d}}{\mathrm{d} t} \frac{\partial}{\partial b}\left(\overline{x_{j}} \circ \rho_{\xi, p}\right)(-t, 0, t) \\
& =-\frac{\partial}{\partial a}\left(\frac{\partial}{\partial b}\left(\overline{x_{j}} \circ \rho_{\xi, p}\right)\right)(-t, 0, t)+\frac{\partial}{\partial c}\left(\frac{\partial}{\partial b}\left(\overline{x_{j}} \circ \rho_{\xi, p}\right)\right)(-t, 0, t),
\end{aligned}
$$

onde foi usada a regra da cadeia.

$$
\begin{aligned}
\frac{\partial}{\partial b}\left(\overline{x_{j}} \circ \rho_{\xi, p}\right) & =\mathrm{d} \rho_{\xi, p}\left(\left.\frac{\partial}{\partial b}\right|_{(a, b, c)}\right)\left[\left.\overline{x_{j}}\right|_{\rho_{\xi, p}(a, b, c)}\right] \\
= & \frac{\partial \rho_{\xi, p}}{\partial b}(a, b, c)\left[\left.\overline{x_{j}}\right|_{\rho_{\xi, p}}\right]=\mathrm{d} X_{a}\left(\left.Y\right|_{Y_{b} X_{c}(p)}\right)\left[\left.\overline{x_{j}}\right|_{\rho_{\xi, p}}\right] \\
& =\left.Y\right|_{Y_{b} X_{c}(p)}\left[\left.\left(\widetilde{x_{j}} \circ X_{a}\right)\right|_{Y_{b} X_{c}(p)}\right]
\end{aligned}
$$


onde $\widetilde{x_{j}}$ é a restrição de $\overline{x_{j}}$ ao contra-domínio do difeomorfismo local $X_{a}$.

$$
\begin{array}{r}
\frac{\partial}{\partial c}\left(\frac{\partial}{\partial b}\left(\overline{x_{j}} \circ \rho_{\xi, p}\right)\right)(-t, 0, t)=\left.\frac{\mathrm{d}}{\mathrm{d} c}\right|_{t}\left(\frac{\partial}{\partial b}\left(\overline{x_{j}} \circ \rho_{\xi, p}\right)(-t, 0, c)\right) \\
=\left.\frac{\mathrm{d}}{\mathrm{d} c}\right|_{t}\left(\left.Y\right|_{X_{c}(p)}\left[\left.\left(\widetilde{x_{j}} \circ X_{-t}\right)\right|_{X_{c}(p)}\right]\right)=\left.\frac{\mathrm{d}}{\mathrm{d} c}\right|_{t}\left(\widetilde{Y}\left(\widetilde{x_{j}} \circ X_{-t}\right)\left(X_{c}(p)\right)\right) \\
=\left.\frac{\mathrm{d}}{\mathrm{d} c}\right|_{t}\left(\widetilde{Y}\left(\widetilde{x_{j}} \circ X_{-t}\right)(\alpha(c))\right),
\end{array}
$$

onde $\tilde{Y}$ é a restrição do campo $Y$ ao domínio de $X_{-t}$ e $\alpha(c)=X_{c}(p)$ é uma curva integral de $X$, com o domínio devidamente escolhido para que sua imagem esteja dentro do domínio de $X_{-t}$. Assim, temos:

$$
\begin{aligned}
\left.\frac{\mathrm{d}}{\mathrm{d} c}\right|_{t}\left(\widetilde{Y}\left(\widetilde{x_{j}} \circ X_{-t}\right)(\alpha(c))\right) & =\alpha^{\prime}(t)\left[\left.\widetilde{Y}\left(\widetilde{x_{j}} \circ X_{-t}\right)\right|_{\alpha(t)}\right] \quad\left(\text { definição de } \alpha^{\prime}\right) \\
& =\left.X\right|_{X_{t}(p)}\left[\left.\widetilde{Y}\left(\widetilde{x_{j}} \circ X_{-t}\right)\right|_{X_{t}(p)}\right] \\
& =\widetilde{X} \tilde{Y}\left(\left(\widetilde{x_{j}} \circ X_{-t}\right)\right)\left(X_{t}(p)\right) \\
\therefore \frac{\partial}{\partial c}\left(\frac{\partial}{\partial b}\left(\overline{x_{j}} \circ \rho_{\xi, p}\right)\right) & (-t, 0, t)=\widetilde{X} \tilde{Y}\left(\left(\widetilde{x_{j}} \circ X_{-t}\right)\right)\left(X_{t}(p)\right)
\end{aligned}
$$

onde $\widetilde{X}$ é a restrição apropriada do campo $X$.

Para calcular

$$
\frac{\partial}{\partial a}\left(\frac{\partial}{\partial b}\left(\overline{x_{j}} \circ \rho_{\xi, p}\right)(-t, 0, t)\right)(-t, 0, t),
$$

vamos inverter a ordem da derivação:

$$
\begin{aligned}
& \frac{\partial}{\partial a}\left(\overline{x_{j}} \circ \rho_{\xi, p}\right)=\frac{\partial \rho_{\xi, p}}{\partial a}(a, b, c)\left[\left.\overline{x_{j}}\right|_{\rho_{\xi, p}}\right]=\left.X\right|_{\rho_{\xi, p}}\left[\left.\overline{x_{j}}\right|_{\rho_{\xi, p}}\right]=\left(X \overline{x_{j}}\right) \circ \rho_{\xi, p} . \\
& \frac{\partial}{\partial b}\left(\frac{\partial}{\partial a}\left(\overline{x_{j}} \circ \rho_{\xi, p}\right)\right)(-t, 0, t)=\left.\frac{\mathrm{d}}{\mathrm{d} b}\right|_{0}\left(\frac{\partial}{\partial a}\left(\overline{x_{j}} \circ \rho_{\xi, p}\right)(-t, b, t)\right) \\
&=\left.\frac{\mathrm{d}}{\mathrm{d} b}\right|_{0} X\left(\overline{x_{j}}\right)\left(X_{-t} Y_{b} X_{t}(p)\right)=\left.\frac{\mathrm{d}}{\mathrm{d} b}\right|_{0} X\left(\overline{x_{j}}\right) \circ \rho_{\xi, p}(-t, b, t) \\
&=\left.\frac{\partial}{\partial b}\right|_{(-t, 0, t)}\left[\left.\left(X\left(\overline{x_{j}}\right) \circ \rho_{\xi, p}\right)\right|_{(-t, 0, t)}\right] \\
&=\frac{\partial \rho_{\xi, p}}{\partial b}(-t, 0, t)\left[\left.\left(X\left(\overline{x_{j}}\right)\right)\right|_{p}\right]=\mathrm{d} X_{-t}\left(\left.Y\right|_{X_{t}(p)}\right)\left[\left.X\left(\overline{x_{j}}\right)\right|_{p}\right] \\
&=\left.\tilde{Y}\right|_{X_{t}(p)}\left[\left.\left(\widetilde{X} \widetilde{x_{j}} \circ X_{-t}\right)\right|_{X_{t}(p)}\right]=\tilde{Y}\left(\widetilde{X} \widetilde{x_{j}} \circ X_{-t}\right)\left(X_{t}(p)\right) .
\end{aligned}
$$




$$
\therefore \frac{\partial}{\partial b}\left(\frac{\partial}{\partial a}\left(\overline{x_{j}} \circ \rho_{\xi, p}\right)\right)(-t, 0, t)=\tilde{Y}\left(\widetilde{X} \widetilde{x_{j}} \circ X_{-t}\right)\left(X_{t}(p)\right) .
$$

Assim, por (2.76), (2.77) e (2.78),

$$
w_{j}^{\prime}(t)=\tilde{X}\left(\tilde{Y}\left(\widetilde{x}_{j} \circ X_{-t}\right)\right)\left(X_{t}(p)\right)-\tilde{Y}\left(\tilde{X}{\widetilde{x_{j}}}_{X_{-t}}\right)\left(X_{t}(p)\right) .
$$

Mostremos que $\left.\tilde{X}{\widetilde{x_{j}}}_{X_{-t}}\right|_{X_{t}(p)}=\left.\tilde{X}\left(\widetilde{x_{j}} \circ X_{-t}\right)\right|_{X_{t}(p)}$.

$$
\begin{aligned}
\widetilde{X}\left(\widetilde{x_{j}} \circ X_{-t}\right)(q) & =\left.\widetilde{X}\right|_{q}\left[\left.\left(\widetilde{x_{j}} \circ X_{-t}\right)\right|_{q}\right]=\mathrm{d} X_{-t}\left(\left.\widetilde{X}\right|_{q}\right)\left[\left.\widetilde{x_{j}}\right|_{X_{-t}(q)}\right] \\
& =\mathrm{d} X_{-t}\left(\left.\frac{\mathrm{d}}{\mathrm{d} s}\right|_{0} \widetilde{X}_{s}(q)\right)=\left.\frac{\mathrm{d}}{\mathrm{d} s}\right|_{0} X_{-t}\left(\widetilde{X_{s}}(q)\right)\left[\left.\widetilde{x_{j}}\right|_{X_{-t}(q)}\right] \\
& =\left.\frac{\mathrm{d}}{\mathrm{d} s}\right|_{0} X_{s-t}(q)\left[\left.\widetilde{x_{j}}\right|_{X_{-t}(q)}\right] \quad\left(\text { para } q \text { próximo de } X_{t}(p)\right) \\
& =\left.X\right|_{X_{-t}(q)}\left[\left.\widetilde{x_{j}}\right|_{X_{-t}(q)}\right]=\widetilde{X} \widetilde{x}_{j}\left(X_{-t}(q)\right)=\left(\widetilde{X} \widetilde{x_{j}} \circ X_{-t}\right)(q) .
\end{aligned}
$$

Assim, usando (2.79) temos:

$$
\begin{aligned}
w_{j}^{\prime}(t) & =\tilde{X}\left(\widetilde{Y}\left(\widetilde{x_{j}} \circ X_{-t}\right)\right)\left(X_{t}(p)\right)-\widetilde{Y}\left(\widetilde{X}\left(\widetilde{x_{j}} \circ X_{-t}\right)\right)\left(X_{t}(p)\right) \\
& =(\tilde{X} \widetilde{Y}-\widetilde{Y} \widetilde{X})\left(\widetilde{x_{j}} \circ X_{-t}\right)\left(X_{t}(p)\right)=[\widetilde{X}, \widetilde{Y}]\left(\widetilde{x_{j}} \circ X_{-t}\right)\left(X_{t}(p)\right) \\
& =\left.[X, Y]\right|_{X_{t}(p)}\left[\left.\left(\widetilde{x_{j}} \circ X_{-t}\right)\right|_{X_{t}(p)}\right]=\mathrm{d} X_{-t}\left(\left.[X, Y]\right|_{X_{t}(p)}\right)\left[\left.x_{j}\right|_{p}\right] . \\
\therefore W^{\prime}(t) & =\left.\sum_{j=1}^{m} w_{j}^{\prime}(t) \frac{\partial}{\partial x_{j}}\right|_{p}=\left.\sum_{j=1}^{m} \mathrm{~d} X_{-t}\left(\left.[X, Y]\right|_{X_{t}(p)}\right)\left[\left.x_{j}\right|_{p}\right] \frac{\partial}{\partial x_{j}}\right|_{p} \\
& =\mathrm{d} X_{-t}\left(\left.[X, Y]\right|_{X_{t}(p)}\right) .
\end{aligned}
$$

Teorema 2.80 (Teorema 4.2 de Sussmann [10]). Sejam $M$ uma variedade $\mathcal{C}^{\infty}$ e $\Delta$ uma distribuição $\mathcal{C}^{\infty}$ (em $M$ ) gerada por uma familia $D$ de campos vetoriais definida em todo lugar. As seguintes condições são equivalentes:

(a) $\Delta$ tem a propriedade das subvariedades integrais.

(b) $\Delta$ tem a propriedade das subvariedades integrais maximais.

(c) $\Delta$ é D-invariante.

(d) $\forall X \in D, t \in \mathbb{R}, p \in M$ tais que $X_{t}(p)$ é definido, $\mathrm{d} X_{t}$ leva vetores de $\Delta(p)$ em vetores de $\Delta\left(X_{t}(p)\right)$. 
(e) $\Delta$ tem posto constante nas D-órbitas e, para cada $p \in M$, existem $X^{1}, \ldots, X^{k} \in D$ tais que:

(1) $\Delta(p)$ é gerado por $\left.X^{1}\right|_{p}, \ldots,\left.X^{k}\right|_{p} e$

(2) para cada $X \in D$ existe $\epsilon>0$ tal que existem funções $\mathcal{C}^{\infty}$

$$
f_{j}^{i}:(-\epsilon, \epsilon) \rightarrow \mathbb{R}, \quad i, j=1, \ldots, k
$$

que satisfazem

$$
\left.\left[X, X^{i}\right]\right|_{X_{t}(p)}=\left.\sum_{j=1}^{k} f_{j}^{i}(t) X^{j}\right|_{X_{t}(p)}, \quad i=1, \ldots, k .
$$

(f) $\Delta=P_{D}$

Demonstração. As implicações

$$
(\mathrm{d}) \Rightarrow(\mathrm{c}) \Rightarrow(\mathrm{f}) \Rightarrow(\mathrm{b}) \Rightarrow(\mathrm{a})
$$

são consequência direta do teorema 2.73 e das definições usadas. Mostremos então as implicações (a) $\Rightarrow(\mathrm{e}) \Rightarrow(\mathrm{d})$.

$(\mathbf{a}) \Rightarrow(\mathbf{e})$ : Suponha que $\Delta$ tem a propriedade das subvariedades integrais.

1. Afirmação: $\Delta$ tem posto constante nas $D$-órbitas. Para mostrar isto, basta mostrar que o posto de $\Delta$ é constante sobre as curvas integrais de cada campo $X \in D$. Seja $\alpha:(a, b) \rightarrow M$ uma destas curvas integrais. Se mostrarmos que o posto de $\Delta$ é localmente constante sobre $\alpha$, isto é, que para todo $t \in(a, b)$ existe $\delta>0$ tal que $|h|<\delta \Rightarrow$ $\operatorname{dim} \Delta(\alpha(t+h))=\operatorname{dim} \Delta(\alpha(t))$, isto será suficiente.

Ora, pegue uma subvariedade integral $A$ de $\Delta$ contendo o ponto $p$. Como $\alpha$ é curva integral de um campo tangente a $A, \alpha(t+h)$ estará em $A$ para todo $h$ suficientemente pequeno (coincidindo com a curva integral de $\left.X\right|_{A}$ que passa por $\alpha(t)$ quando o parâmetro é $t$ ). Como a dimensão de $A$ é sempre a mesma (por se tratar de uma variedade),

$$
\operatorname{dim} \Delta(\alpha(t+h))=\operatorname{dim} A=\operatorname{dim} \Delta(\alpha(t)) .
$$

2. Dado $p \in M$, seja $N$ uma subvariedade integral de $\Delta$ contendo o ponto $p$. Sejam $X^{1}, \ldots, X^{k} \in D$ tais que $\left.X^{1}\right|_{p}, \ldots,\left.X^{k}\right|_{p}$ são uma base de $\Delta(p)$. Como eles são tangentes a $N$, considere as restrições $Y^{1}=$ 
$\left.X^{1}\right|_{N}, \ldots, Y^{k}=\left.X^{k}\right|_{N}$. Os vetores $\left.Y^{1}\right|_{p}, \ldots,\left.Y^{k}\right|_{p}$ formam uma base de $\mathrm{T}_{p} N$. Pela continuidade dos campos, existe uma vizinhança aberta $V \subset N$ de $p$ tal que, para todo $p^{\prime} \in V,\left.Y^{1}\right|_{p^{\prime}}, \ldots,\left.Y^{k}\right|_{p^{\prime}}$ são base de $\mathrm{T}_{p^{\prime}} N$.

Podemos supor, pela escolha adequada de $N$, que $N=V$. Assim, os campos $Y^{1}, \ldots, Y^{k}$ formam uma base do espaço tangente de $N$ em cada ponto. Isto implica que, dado qualquer campo vetorial real $W \in \mathfrak{X}(N)$, podemos escrever $W=\sum_{j=1}^{k} w_{j} Y^{j}$, onde as funções $w_{j}: V \rightarrow \mathbb{R}$ serão suaves - para verificar isto, basta verificar que a matriz mudança de base, das derivadas parciais das cartas locais de $V$ para os campos $Y^{1}, \ldots, Y^{k}$, será suave.

Tome estes campos $X^{1}, \ldots, X^{k}$ como os requeridos pela condição (e). Dado $X \in D$,

$$
\left[X, X^{i}\right] \text { é tangente a } N .
$$

Assim, podemos escrever

$$
\left.\left[X, X^{i}\right]\right|_{N}=\sum_{j=1}^{k} g_{j}^{i} Y^{j}, \quad \forall i=1, \ldots, k
$$

onde as funções $g_{j}^{i}: i, j=1, \ldots, k$ são suaves em $N$.

Seja $\delta>0$ tal que $X_{t}(p) \in N$ sempre que $|t|<\delta$. Assim, temos

$$
\left.\left[X, X^{i}\right]\right|_{X_{t}(p)}=\left.\sum_{j=1}^{k} g_{j}^{i}\left(X_{t}(p)\right) X^{j}\right|_{X_{t}(p)}, \quad \forall i=1, \ldots, k .
$$

As funções $t \mapsto g_{j}^{i}\left(X_{t}(p)\right)$ são as $f_{j}^{i}$ requeridas na condição (e).

$(\mathbf{e}) \Rightarrow(\mathrm{d})$ : Suponha que vale a propriedade $(\mathrm{e})$. Chamemos de $\left(\mathrm{d}^{\prime}\right)$ a seguinte propriedade: para cada $p \in M, X \in D, \exists \delta>0$ tal que $\forall|t|<\delta$ : $\mathrm{d} X_{t}(\Delta(p))=\Delta\left(X_{t}(p)\right)$. Mostraremos então que a condição (e) implica $\left(\mathrm{d}^{\prime}\right)$ e que $\left(d^{\prime}\right)$ implica $(d)$.

$(\mathbf{e}) \Rightarrow(\mathbf{d})^{\prime}$ : Defina, para $|t|<\epsilon, W_{i}(t)=\mathrm{d} X_{-t}\left(\left.X^{i}\right|_{X_{t}(p)}\right)$. Então, pelo lema 2.71,

$$
\begin{aligned}
W_{i}^{\prime}(t) & =\mathrm{d} X_{-t}\left(\left.\left[X, X^{i}\right]\right|_{X_{t}(p)}\right) \\
& =\sum_{j=1}^{k} f_{j}^{i}(t) \mathrm{d} X_{-t}\left(\left.X^{j}\right|_{X_{t}(p)}\right)=\sum_{j=1}^{k} f_{j}^{i}(t) W_{j}(t) .
\end{aligned}
$$


Assim, $\left(W_{1}, \ldots, W_{k}\right)$, curva em $\left(\mathrm{T}_{p} M\right)^{k}$, é uma solução do sistema de EDO:

$$
\begin{cases}W_{i}^{\prime}(t)=\sum_{j=1}^{k} f_{j}^{i}(t) W_{j}(t), & i=1, \ldots, k \\ W_{i}(0)=\left.X^{i}\right|_{p}, & i=1, \ldots, k\end{cases}
$$

Como as condições iniciais de $W_{1}, \ldots, W_{k}$ estão em $\Delta(p)$, então os vetores $W_{1}(t), \ldots, W_{k}(t)$ estarão em $\Delta(p)$ para todo $|t|<\epsilon$. Não mostraremos isto em detalhes. A ideia é que podemos resolver o sistema acima no espaço $\Delta(p)^{k}$ e usar a unicidade da solução.

Como $W_{1}(0), \ldots, W_{k}(0)$ são base $W_{1}, \ldots, W_{k}$, existe $\delta \in(0, \epsilon)$ tal que $W_{1}(t), \ldots, W_{k}(t)$ geram $\Delta(p)$ para todo $|t|<\delta$.

Isto implica que todo vetor $\vec{v} \in \Delta(p)$ pode ser escrito como combinação linear dos $W_{i}(t)$, para todo $|t|<\delta$. Mas

$$
\mathrm{d} X_{t}\left(W_{i}(t)\right)=\left.X^{i}\right|_{X_{t}(p)} \in \Delta\left(X_{t}(p)\right)
$$

Assim, $\mathrm{d} X_{t}(\Delta(p)) \subset \Delta\left(X_{t}(p)\right), \forall|t|<\delta$.

Como $X_{t}$ é um difeomorfismo local, a dimensão de $\mathrm{d} X_{t}(\Delta(p))$ é a mesma que a dimensão de $\Delta(p)$, que é a mesma que a dimensão de $\Delta\left(X_{t}(p)\right)$ pois, por hipótese, o posto de $\Delta$ é constante nas órbitas. Assim $\mathrm{d} X_{t}(\Delta(p))=$ $\Delta\left(X_{t}(p)\right)$, sempre que $|t|<\delta$.

$\left(\mathbf{d}^{\prime}\right) \Rightarrow(\mathbf{d})$ : Agora, devemos mostrar que vale $\mathrm{d} X_{t}(\Delta(p)) \subset \Delta\left(X_{t}(p)\right)$ para todo $t$ em que $X_{t}(p)$ é definido.

Seja $(a, b) \subset \mathbb{R}$ o domínio da curva integral maximal $t \mapsto X_{t}(p)$. Seja $J \subset$ $\left(a_{p}, b_{p}\right)$ o conjunto de todos os $t$ tais que $\mathrm{d} X_{t}(\Delta(p))=\Delta\left(X_{t}(p)\right)$. Devemos mostrar que $J=(a, b)$.

Dado $t \in J \operatorname{com} q=X_{t}(p)$, pela propriedade $(\mathrm{d})^{\prime}$, existe $\delta_{t}>0$ tal que

$$
|h|<\delta_{t} \Rightarrow \mathrm{d} X_{h}(\Delta(q))=\Delta\left(X_{h}(q)\right) .
$$

Pela propriedade de grupo (proposição 2.13) e pela regra da cadeia,

$$
X_{h}(q)=X_{t+h}(p) \text { e } \mathrm{d} X_{t+h}(\Delta(p))=\mathrm{d} X_{h}\left(\mathrm{~d} X_{t}(\Delta(p))\right)=\Delta\left(X_{t+h}(p)\right) .
$$

Assim, $\left(t-\delta_{t}, t+\delta_{t}\right) \in J$. Portanto, $J$ é aberto.

$J$ é fechado em $(a, b)$ : Seja $\left(t_{0}, t_{1}, \ldots\right)$ uma sequência em $J$ que converge para $t \in(a, b)$. Afirmação: $t \in J$. De fato, seja $\delta^{\prime}$ tal que

$$
|h|<\delta^{\prime} \Rightarrow \mathrm{d} X_{h}\left(\Delta\left(X_{t}(p)\right)\right)=\Delta\left(X_{h}\left(X_{t}(p)\right)\right)=\Delta\left(X_{t+h}(p)\right)
$$


(dado pela propriedade $\left(\mathrm{d}^{\prime}\right)$ ). Como $t$ é o limite de uma sequência em $J$, existe $t_{k} \in J$ tal que $\left|t-t_{k}\right|<\delta_{t}$. Então $X_{t_{k}}(p)=X_{h}\left(X_{t}(p)\right)$, para $h=t-t_{k}$. Assim, $X_{t}(p)=X_{-h} X_{t_{k}}(p)$ e

$$
\mathrm{d} X_{t}(\Delta(p))=\mathrm{d} X_{-h}\left(\mathrm{~d} X_{t_{k}}(\Delta(p))=\mathrm{d} X_{-h}\left(\Delta\left(X_{t_{k}}(p)\right)\right) .\right.
$$

Como $X_{-h}$ é o inverso de $X_{h}, \mathrm{~d} X_{-h}$ é o inverso de $\mathrm{d} X_{h}$ e, assim, leva $\Delta\left(X_{t_{k}}(p)\right)$ em $\Delta\left(X_{t}(p)\right)$. Portanto, $\mathrm{d} X_{t}(\Delta(p))=\Delta\left(X_{t}(p)\right)$ e $t \in J$.

Temos que $J$ é aberto e fechado em $(a, b)$, portanto $J=(a, b)$.

Corolário 2.81. Uma distribuição suave $\Delta=\Delta_{D}$ em $M$ é D-invariante se e somente se ela é involutiva e seu posto é constante nas D-órbitas.

Demonstração. Se $\Delta$ é $D$-invariante, $\Delta=P_{D}$ e o teorema 2.73 nos diz que $P_{D}$ é involutiva e tem posto constante nas $D$-órbitas.

Por outro lado, se $\Delta$ é involutiva e tem posto constante nas órbitas, vamos mostrar que $\Delta$ satisfaz a condição (e) do teorema 2.80 .

Dado $p \in M, \operatorname{sejam} X^{1}, \ldots, X^{k} \in D$ tais que $\left.X^{1}\right|_{p}, \ldots,\left.X^{k}\right|_{p}$ são uma base de $\Delta(p)$. Dado $X \in D$ qualquer, considere a curva integral $\alpha(t)=X_{t}(p)$. Seja $\epsilon>0$ tal que $\left.X^{1}\right|_{\alpha(t)}, \ldots,\left.X^{k}\right|_{\alpha(t)}$ são L.I. para todo $|t|<\epsilon$.

Como o posto de $\Delta$ é constante nas órbitas,

$$
\operatorname{dim} \Delta(\alpha(t))=\operatorname{dim} \Delta(\alpha(0))=k .
$$

Isto implica que $\left.X^{1}\right|_{\alpha(t)}, \ldots,\left.X^{k}\right|_{\alpha(t)}$ é uma base de $\Delta(\alpha(t))$ sempre que $|t|<$ $\epsilon$.

Seja $i \in\{1, \ldots, k\}$. Como $\Delta$ é involutiva, $\left[X, X^{i}\right] \in \Delta$. Como os campos $X^{1}, \ldots, X^{k}$ formam uma base de $\Delta$ sobre $\alpha(t),|t|<\epsilon$, podemos escrever

$$
\left.\left[X, X^{i}\right]\right|_{\alpha(t)}=\left.\sum_{j=1}^{k} f_{j}^{i}(t) X^{j}\right|_{\alpha(t)}, \quad \forall|t|<\epsilon .
$$

Fica como exercício para o leitor provar que as funções $f_{j}^{i}$ são suaves. 


\section{Capítulo 3}

\section{Um Parêntese: Transformada de Fourier}

\subsection{Aproximação da identidade}

Definição 3.1. Dados $\Omega \subset \mathbb{R}^{n}, p \in[1, \infty)$ e $m^{n}$ a medida de Lebesgue em $\mathbb{R}^{n}$, o espaço $L^{p}(\Omega)$ é o espaço vetorial

$$
\left\{f: \Omega \rightarrow \mathbb{C} \mid f \text { Lebesgue-mensurável e } \int_{\Omega}|f|^{p} \mathrm{~d} m^{n}<\infty\right\},
$$

dotado da seminorma

$$
\|f\|_{p}=\left(\int_{\Omega}|f|^{p} \mathrm{~d} m^{n}\right)^{\frac{1}{p}}
$$

Proposição 3.2. Seja $\phi: \mathbb{R} \rightarrow \mathbb{C}$ uma função $L^{1}$. O majorante radial de $\phi$ é a função

$$
\begin{aligned}
\eta: \mathbb{R}^{+} & \longrightarrow[0, \infty] \\
h & \longmapsto \sup _{|y|>h}|\phi(y)| .
\end{aligned}
$$

Suponha que $I:=\int_{0}^{\infty} \eta(h) \mathrm{d} h$ é finito.

Então, para toda função localmente integrável $f$, dados $x \in \mathbb{R}$ e $M>0$ arbitrários,

$$
\int_{-\infty}^{\infty}\left|\phi_{M}(x) f(x-y)\right| \mathrm{d} y \leq I \mathrm{H} f(x)
$$

onde $\phi_{M}(x)=M \phi(M x)$ e $\mathrm{H} f$ é a função maximal de Hardy-Littlewood, definida por

$$
\mathrm{H} f(x)=\sup _{r>0} \frac{1}{2 r} \int_{-r}^{r}|f(x-y)| \mathrm{d} y .
$$


Demonstração. Fixado $x \in \mathbb{R}$, seja $F=F_{x}: \mathbb{R}^{+} \rightarrow \mathbb{R}$ definida por

$$
F(h)=\int_{-h}^{h}|f(x-y)| \mathrm{d} y .
$$

Note que

$$
F(h) \leq 2 h \mathrm{H} f(x) .
$$

$F$ é contínua e, quando restrita a compactos, é de variação limitada. Assim, dado $r>0$, podemos definir no intervalo [0, $r$ a medida de LebesgueStieltjes $\mu_{F}$, determinada pela fórmula

$$
\mu_{F}([a, b])=F(b)-F(a)=\int_{|y| \in[a, b]}|f(x-y)| \mathrm{d} y,
$$

para todo intervalo $[a, b] \subset[0, r]$. É fácil ver que, para uma função integrável $g:[0, r] \rightarrow \mathbb{C}$, a integral de Lebesgue-Stieltjes de $g$ em relação a $F$ será

$$
\int_{0}^{r} g(h) \mathrm{d} F(h)=\int_{-r}^{r} g(|h|)|f(x-h)| \mathrm{d} h .
$$

Assim,

$$
\int_{-r}^{r}\left|\phi_{M}(y) f(x-y)\right| \mathrm{d} y \leq \int_{-r}^{r} \eta_{M}(|y|)|f(x-y)| \mathrm{d} y=\int_{0}^{r} \eta_{M}(y) \mathrm{d} F(y),
$$

onde $\eta_{M}(y)=M \eta(M y)$. A função $\eta_{M}$ tem variação limitada em [0,r]. Com isto e com o fato de que $F$ tem variação limitada em $[0, r]$ e é contínua, podemos fazer a seguinte integração por partes:

$$
\begin{gathered}
\int_{0}^{r} \eta_{M}(y) \mathrm{d} F(y)=\eta_{M}(r) F(r)-\int_{0}^{r} F(y) \mathrm{d} \eta_{M}(y) \\
-\int_{0}^{r} F(y) \mathrm{d} \eta_{M}(y)=\int_{0}^{r} F(y)\left(-\mathrm{d} \eta_{M}(y)\right) \leq \int_{0}^{r} 2 y \mathrm{H} f(x)\left(-\mathrm{d} \eta_{M}(y)\right) \\
=2 \mathrm{H} f(x) \int_{0}^{r}-y \mathrm{~d} \eta_{M}(y)
\end{gathered}
$$

(notando que $-d \eta_{M}(y)$ é medida positiva). Novamente, usamos a integração por partes para calcular

$$
\int_{0}^{r}-y \mathrm{~d} \eta_{M}(y)=\eta_{M}(r)(-r)+\int_{0}^{r} \eta_{M}(y) \mathrm{d} y .
$$


Temos

$$
\begin{aligned}
\int_{-r}^{r}\left|\phi_{M}(y) f(x-y)\right| \mathrm{d} y & \\
& \leq \eta_{M}(r) F(r)+2 \mathrm{H} f(x)\left(\eta_{M}(r)(-r)+\int_{0}^{r} \eta_{M}(y) \mathrm{d} y\right) .
\end{aligned}
$$

Note que $\lim _{r \rightarrow \infty} \eta_{M}(r) r=0$, pois

$$
\int_{\frac{r}{2}}^{r} \eta_{M}(y) \mathrm{d} y \geq \eta_{M}(r) \frac{r}{2} \quad \text { e } \quad \lim _{r \rightarrow \infty} \int_{\frac{r}{2}}^{r} \eta_{M}(y) \mathrm{d} y=0 .
$$

Assim, fazendo $r \rightarrow \infty$, obtemos

$$
\int_{-\infty}^{\infty}\left|\phi_{M}(y) f(x-y)\right| \mathrm{d} y \leq 2 \mathrm{H} f(x) \int_{0}^{\infty} \eta_{M}(y) \mathrm{d} y=I \mathrm{H} f(x) .
$$

Corolário 3.3. Sejam $f \in L^{1}(\mathbb{R})$ e $\phi \in L^{1}(\mathbb{R})$ tais que $\phi$ tem majorante radial integrável e $I:=\int \phi$. Então, para quase todo $x \in \mathbb{R}$,

$$
\lim _{M \rightarrow \infty} f * \phi_{M}(x)=\operatorname{If}(x) .
$$

Demonstração. Basta mostrar o caso em que $I=1$. Primeiro, suponha que $f \in \mathcal{C}_{c}^{\infty}(\mathbb{R})$. Seja $v(y)=\int_{-\infty}^{y} \phi_{M}(t) \mathrm{d} t$. Façamos uma integração por partes:

$$
\int_{-\infty}^{\infty} f(x-y) \phi_{M}(y) \mathrm{d} y=\int_{-\infty}^{\infty} f(x-y) \mathrm{d} v(y)=\int_{-\infty}^{\infty} v(y) f^{\prime}(x-y) \mathrm{d} y .
$$

Como $\lim _{M \rightarrow \infty} v(y)$ é 0 se $y<0$ e 1 se $y>0$,

$$
\int_{-\infty}^{\infty} v(y) f^{\prime}(x-y) \mathrm{d} y \stackrel{M \rightarrow \infty}{\longrightarrow} \int_{0}^{\infty} f^{\prime}(x-y) \mathrm{d} y=f(x) .
$$

Agora, suponha que $f$ é uma função integrável qualquer. Usando o fato de que as funções suaves de suporte compacto são densas em $L^{1}(\mathbb{R})$, vamos escrever

$$
f=f_{0}+r
$$

onde $f_{0} \in \mathcal{C}_{c}^{\infty}(\mathbb{R})$ e $\|r\|_{1}$ é arbitrariamente pequena.

$$
\begin{aligned}
& \left|f * \phi_{M}(x)-f(x)\right|=\left|\left(f_{0}+r\right) * \phi_{M}(x)-\left(f_{0}+r\right)(x)\right| \\
& \quad=\left|f_{0} * \phi_{M}(x)+r * \phi_{M}(x)-f_{0}(x)+r(x)\right| \\
& \leq\left|f_{0} * \phi_{M}(x)-f_{0}(x)\right|+\left|r * \phi_{M}(x)\right|+|r(x)| \\
& \quad \leq\left|f_{0} * \phi_{M}(x)-f_{0}(x)\right|+J \mathrm{H} r(x)+|r(x)|
\end{aligned}
$$


onde $J$ é a integral do majorante radial de $\phi$.

Dado $\epsilon>0$, temos:

1. $|r(x)|<\epsilon$ para todo $x$ exceto em um conjunto de medida menor que $\|r\|_{1} / \epsilon$.

2. $\mathrm{H} r(x)<\epsilon$ para todo $x$ exceto em um conjunto de medida menor que $C\|r\|_{1} / \epsilon$, onde $C$ é uma constante que não depende da função $r$ (ver Stein [8], capítulo I para uma demonstração deste fato).

Assim,

$$
\limsup _{M \rightarrow \infty}\left|f * \phi_{M}(x)-f(x)\right| \leq(1+J) \epsilon
$$

para todo $x$ exceto em um conjunto de medida menor que

$$
\frac{\|r\|_{1}}{\epsilon}+C \frac{\|r\|_{1}}{\epsilon}
$$

Fazendo $r \rightarrow 0$, reduzimos o conjunto de exceções para medida nula. Fazendo $\epsilon \rightarrow 0$, obtemos o resultado desejado.

\subsection{Transformada de Fourier em $L^{1}(\mathbb{R})$}

Definição 3.4. Seja $f \in L^{1}(\mathbb{R})$. A transformada de Fourier de $f$ é a função $\hat{f}: \mathbb{R} \rightarrow \mathbb{C}$ definida por

$$
\hat{f}(\xi)=\int_{-\infty}^{\infty} \mathrm{e}^{-i \xi x} f(x) \mathrm{d} x .
$$

A transformada de Fourier é um operador linear que será denotado de duas maneiras:

(i) $\hat{f}=(f)^{\wedge}$

(ii) $\hat{f}=\mathcal{F}_{x}(f(x))$

Proposição 3.5. Sejam $f \in L^{1}(\mathbb{R}), \xi \in \mathbb{R}$. Então:

(i) $|\hat{f}(\xi)| \leq\|f\|_{1}$.

(ii) $\mathcal{F}_{x}(f(x+h))(\xi)=\mathrm{e}^{i \xi h} \hat{f}(\xi), \quad \forall h \in \mathbb{R}$.

(iii) $\hat{f}(\xi+h)=\mathcal{F}_{x}\left(\mathrm{e}^{-i x h} f(x)\right)(\xi), \quad \forall h \in \mathbb{R}$. 
(iv) Dado $\lambda \in \mathbb{R}$ diferente de zero,

$$
\mathcal{F}_{x}(f(\lambda x))(\xi)=\frac{1}{|\lambda|} \hat{f}\left(\frac{1}{\lambda} \xi\right)
$$

(v) $\hat{f}$ é uniformemente contínua.

(vi) Se $f \in \mathcal{C}^{1}(\mathbb{R}), f^{\prime} \in L^{1}(\mathbb{R})$ e $\lim _{|x| \rightarrow \infty} f(x)=0$, então

$$
\widehat{f^{\prime}}(\xi)=i \xi \hat{f}(\xi)
$$

(vii) Se a função $x f(x)$ em $x$ pertence a $L^{1}(\mathbb{R})$, então $\hat{f} \in \mathcal{C}^{1}(\mathbb{R})$ e

$$
(\hat{f})^{\prime}=\mathcal{F}_{x}(-i x f(x)) .
$$

(viii) $\lim _{|\xi| \rightarrow \infty} \hat{f}(\xi)=0$.

Demonstração. (i):

$$
\begin{aligned}
&|\hat{f}(\xi)|=\left|\int_{-\infty}^{\infty} \mathrm{e}^{-i \xi x} f(x) \mathrm{d} x\right| \leq \int_{-\infty}^{\infty}\left|\mathrm{e}^{-i \xi x} f(x)\right| \mathrm{d} x=\int_{-\infty}^{\infty}|f(x)| \mathrm{d} x \\
&=\|f\|_{1}
\end{aligned}
$$

(ii):

$$
\begin{aligned}
\mathcal{F}_{x}(f(x+h))=\int_{-\infty}^{\infty} \mathrm{e}^{-i \xi x} f(x+h) \mathrm{d} x & =\int_{-\infty}^{\infty} \mathrm{e}^{-i \xi(u-h)} f(u) \mathrm{d} u \\
=\int_{-\infty}^{\infty} \mathrm{e}^{-i \xi(-h)} \mathrm{e}^{-i \xi u} f(u) \mathrm{d} u & =\mathrm{e}^{i \xi h} \int_{-\infty}^{\infty} \mathrm{e}^{-i \xi u} f(u) \mathrm{d} u=\mathrm{e}^{i \xi h} \hat{f}(\xi) .
\end{aligned}
$$

(iii):

$$
\begin{aligned}
\hat{f}(\xi+h)=\int_{-\infty}^{\infty} \mathrm{e}^{-i(\xi+h) x} f(x) \mathrm{d} x=\int_{-\infty}^{\infty} \mathrm{e}^{-i \xi x} \mathrm{e}^{-i h x} f(x) \mathrm{d} x & \\
& =\mathcal{F}_{x}\left(\mathrm{e}^{-i h x} f(x)\right)(\xi) .
\end{aligned}
$$

(iv): Se $\lambda>0$ :

$$
\int_{-\infty}^{\infty} \mathrm{e}^{-i \xi x} f(\lambda x) \mathrm{d} x=\int_{-\infty}^{\infty} \mathrm{e}^{-i \xi \frac{u}{\lambda}} f(u) \frac{1}{\lambda} \mathrm{d} u=\frac{1}{\lambda} \hat{f}\left(\frac{\xi}{\lambda}\right) .
$$

Se $\lambda<0$ :

$$
\int_{-\infty}^{\infty} \mathrm{e}^{-i \xi x} f(\lambda x) \mathrm{d} x=\int_{\infty}^{-\infty} \mathrm{e}^{-i \xi \frac{u}{\lambda}} f(u) \frac{1}{\lambda} \mathrm{d} u=-\frac{1}{\lambda} \hat{f}\left(\frac{\xi}{\lambda}\right) .
$$


(v):

$$
\begin{aligned}
\mid \hat{f}(\xi+h)- & \hat{f}(\xi)|=| \mathcal{F}_{x}\left(\left(\mathrm{e}^{-i h x} f(x)\right)(\xi)-\hat{f}(\xi) \mid\right. \\
& =\left|\mathcal{F}_{x}\left(\left(\mathrm{e}^{-i h x}-1\right) f(x)\right)(\xi)\right| \leq\left\|x \mapsto\left(\mathrm{e}^{-i h x}-1\right) f(x)\right\|_{1} \stackrel{h \rightarrow 0}{\longrightarrow} 0
\end{aligned}
$$

onde é usado o teorema da convergência dominada, e a convergência não depende da variável $\xi$.

(vi): Suponha que $f^{\prime}, f \in L^{1}(\mathbb{R}), f^{\prime}$ é contínua e $\lim _{|x| \rightarrow \infty} f(x)=0$. Por integração por partes:

$$
\begin{aligned}
\widehat{f^{\prime}}(\xi)=\int_{-\infty}^{\infty} \mathrm{e}^{-i \xi x} f^{\prime}(x) \mathrm{d} x=\lim _{M \rightarrow \infty} \int_{-M}^{M} \mathrm{e}^{-i \xi x} f^{\prime}(x) \mathrm{d} x \\
=\lim _{M \rightarrow \infty}\left[\left.\mathrm{e}^{-i \xi x} f(x)\right|_{x=-M} ^{M}-\int_{-M}^{M}\left(\frac{\mathrm{d}}{\mathrm{d} x} \mathrm{e}^{-i \xi x}\right) f(x) \mathrm{d} x\right] \\
=-\int_{-\infty}^{\infty}(-i \xi) \mathrm{e}^{-i \xi x} f(x) \mathrm{d} x=i \xi \hat{f}(\xi)
\end{aligned}
$$

(vii): Suponha que $x \mapsto x f(x) \in L^{1}(\mathbb{R})$.

$$
\frac{\hat{f}(\xi+h)-\hat{f}(\xi)}{h}=\frac{1}{h} \mathcal{F}_{x}\left(\mathrm{e}^{-i x h} f(x)-f(x)\right)(\xi)=\mathcal{F}_{x}\left(\frac{f(x)\left(\mathrm{e}^{-i x h}-1\right)}{h}\right)(\xi) \text {. }
$$

Pela propriedade (i), se mostrarmos que

$$
\left[x \mapsto f(x) \frac{\mathrm{e}^{-i x h}-1}{h}\right] \stackrel{h \rightarrow 0}{\longrightarrow}[x \mapsto-i x f(x)] \text { em } L^{1}(\mathbb{R}),
$$

então teremos o resultado desejado. A convergência pontual destas funções é dada por:

$$
\lim _{h \rightarrow 0} \frac{\mathrm{e}^{-i x h}-1}{h}=\left.\frac{\mathrm{d}}{\mathrm{d} h}\right|_{0} \mathrm{e}^{-i x h}=-i x(\text { definição de derivada })
$$

Mostremos que a convergência é dominada por $|x f(x)|$. Pela desigualdade do valor médio,

$$
\begin{gathered}
\left|\frac{\mathrm{e}^{-i x h}-1}{h}\right| \leq \sup _{\xi \in[0,1]}\left|\frac{\mathrm{d}}{\mathrm{d} r}\right|_{\xi h} \mathrm{e}^{-i x r} \mid \\
\therefore\left|f(x) \frac{\operatorname{e}^{-i x h}-1}{h}\right| \leq|x f(x)| .
\end{gathered}
$$


Assim, o teorema da convergência dominada nos dá o resultado desejado.

(viii): Suponha inicialmente que $f$ é uma função $\mathcal{C}^{1}$ de suporte compacto. Por (vi), (v) e (i), $\xi \mapsto i \xi \hat{f}(\xi)$ é uma função uniformemente contínua e limitada, o que implica que $\hat{f}( \pm \infty)=0$.

Caso geral: dada $f \in L^{1}(\mathbb{R})$ qualquer, seja $\left(f_{n}\right)_{n \in \mathbb{N}}$ uma sequência de funções $\mathcal{C}^{1}$ de suporte compacto que converge em $L^{1}(\mathbb{R})$ para $f$. Pela propriedade (i), $\hat{f}_{n}$ converge uniformemente para $\hat{f}$. Assim, podemos trocar a ordem no limite duplo:

$$
\hat{f}( \pm \infty)=\lim _{|\xi| \rightarrow \infty} \lim _{n \rightarrow \infty} \hat{f}_{n}(\xi)=\lim _{n \rightarrow \infty} \lim _{|\xi| \rightarrow \infty} \hat{f}_{n}(\xi)=0 .
$$

Exemplo 3.7 (Transformada da curva gaussiana). Seja $g(x)=\mathrm{e}^{-x^{2}}$. As regras da transformada de Fourier e a integral gaussiana nos dão:

$$
\begin{gathered}
\left(g^{\prime}\right)^{\wedge}(\xi)=i \xi \hat{g}(\xi)=\mathcal{F}_{x}(-2 x g(x))=-2 i \mathcal{F}_{x}(-i x g(x))=-2 i(\hat{g})^{\prime}(\xi), \\
\hat{g}(0)=\int_{-\infty}^{\infty} g(x) \mathrm{d} x=\sqrt{\pi}
\end{gathered}
$$

Assim, $\hat{g}$ é solução de:

$$
\left\{\begin{array}{l}
u^{\prime}(\xi)=-\frac{\xi}{2} u(\xi), \forall \xi \in \mathbb{R} . \\
u(0)=\sqrt{\pi}
\end{array}\right.
$$

Resolvendo a equação diferencial, obtemos a solução

$$
\hat{g}(\xi)=\sqrt{\pi} \mathrm{e}^{-\frac{\xi^{2}}{4}}=\sqrt{\pi} g\left(\frac{1}{2} \xi\right) .
$$

Pela regras da transformada de Fourier, pode-se calcular ainda a transformada segunda (notando que $\hat{g} \in L^{1}(\mathbb{R})$ :

$$
\begin{aligned}
\hat{\hat{g}}(x)=\mathcal{F}_{\xi}\left(\sqrt{\pi} g\left(\frac{1}{2} \xi\right)\right)(x)=\sqrt{\pi}(2 \hat{g}(2 x))= & 2 \sqrt{\pi} \hat{g}(2 x) \\
& =2 \sqrt{\pi}\left(\sqrt{\pi} g\left(\frac{1}{2} 2 x\right)\right)=2 \pi g(x) .
\end{aligned}
$$

\section{Convolução}

Definição 3.8. (i) Definimos o operador como sendo a reflexão:

$$
\tilde{f}(x)=f(-x)
$$

(ii) Dado $h \in \mathbb{R}$, definimos o operador translação $\tau_{h}$ como sendo:

$$
\left(\tau_{h} f\right)(x)=f(x-h) .
$$


Definição 3.9. A convolução de duas funções Lebesgue-mensuráveis $f, g$ : $\mathbb{R} \rightarrow \mathbb{C}$ no ponto $x \in \mathbb{R}$ é denotada $(f * g)(x)$ e definida (quando a integral abaixo existir) por

$$
(f * g)(x)=\int_{-\infty}^{\infty} f(x-y) g(y) \mathrm{d} y
$$

Proposição 3.10. Sejam $f, g, f_{1}, f_{2} \in L^{1}(\mathbb{R}), h \in \mathbb{R}, \lambda \in \mathbb{C}$. A convolução satisfaz as seguintes propriedades:

(i) $f * g=g * f$.

(ii) $(f * g)^{\sim}=\tilde{f} * \tilde{g}$.

(iii) $\tau_{h}(f * g)=\left(\tau_{h} f\right) * g$.

(iv) $\left(f_{1}+\lambda f_{2}\right) * g=f_{1} * g+\lambda f_{2} * g$.

Demonstração.

$$
\begin{aligned}
& (f * g)(x)=\int_{-\infty}^{\infty} f(x-y) g(y) \mathrm{d} y=\int_{-\infty}^{\infty} f(u) g(x-u) \mathrm{d} u=(g * f)(x) . \\
& (f * g)^{\sim}(x)=\int_{-\infty}^{\infty} f(-x-y) g(y) \mathrm{d} y=\int_{-\infty}^{\infty} \tilde{f}(x+y) g(y) \mathrm{d} y \\
& =\int_{-\infty}^{\infty} \tilde{f}(x+y) \tilde{g}(-y) \mathrm{d} y=\int_{-\infty}^{\infty} \tilde{f}(x-u) \tilde{g}(u) \mathrm{d} u=\tilde{f} * \tilde{g}(x) . \\
& \tau_{h}(f * g)(x)=(f * g)(x-h)=\int_{-\infty}^{\infty} f(x-h-y) g(y) \mathrm{d} y \\
& =\int_{-\infty}^{\infty}\left(\tau_{h} f\right)(x-y) g(y) \mathrm{d} y=\left(\tau_{h} f\right) * g(x) . \\
& \left(\left(f_{1}+\lambda f_{2}\right) * g\right)(x)=\int_{-\infty}^{\infty}\left(f_{1}(x-y)+\lambda f_{2}(x-y)\right) g(y) \mathrm{d} y \\
& =\int_{-\infty}^{\infty} f_{1}(x-y) g(y) \mathrm{d} y+\lambda \int_{-\infty}^{\infty} f_{2}(x-y) g(y) \mathrm{d} y \\
& =\left(f_{1} * g+\lambda f_{2} * g\right)(x) \text {. }
\end{aligned}
$$


Proposição 3.11. Sejam $f, g \in L^{1}(\mathbb{R})$. Então

(i) $f * g \in L^{1}(\mathbb{R}) \operatorname{com}\|f * g\|_{1} \leq\|f\|_{1}\|g\|_{1} e$

(ii) $\widehat{f * g}=\hat{f} \hat{g}$.

Demonstração. Para mostrar que $f * g \in L^{1}(\mathbb{R})$, usaremos o teorema de Fubini-Tonelli:

$$
\begin{aligned}
& \int_{\infty}^{\infty}|(f * g)(x)| \mathrm{d} x=\int_{-\infty}^{\infty}\left|\int_{-\infty}^{\infty} f(x-y) g(y) \mathrm{d} y\right| \mathrm{d} x \\
& \leq \int_{-\infty}^{\infty}\left(\int_{-\infty}^{\infty}|f(x-y) g(y)| \mathrm{d} y\right) \mathrm{d} x=\int_{-\infty}^{\infty}\left(\int_{-\infty}^{\infty}|f(x-y) g(y)| \mathrm{d} x\right) \mathrm{d} y \\
& =\int_{-\infty}^{\infty}|g(y)|\left(\int_{-\infty}^{\infty}|f(x-y)| \mathrm{d} x\right) \mathrm{d} y=\|f\|_{1}\|g\|_{1}<\infty
\end{aligned}
$$

Façamos a transformada de Fourier de $f * g$.

$$
\widehat{f * g}(\xi)=\int_{-\infty}^{\infty} \mathrm{e}^{-i x \xi}\left(\int_{-\infty}^{\infty} f(x-y) g(y) \mathrm{d} y\right) \mathrm{d} x
$$

Para mudar a ordem da integração, devemos mostrar que

$$
\begin{gathered}
{\left[(x, y) \mapsto \mathrm{e}^{-i x \xi} f(x-y) g(y)\right] \in L^{1}\left(\mathbb{R}^{2}\right) .} \\
\int_{\mathbb{R}^{2}}\left|\mathrm{e}^{i x \xi} f(x-y) g(y)\right| \mathrm{d} m^{2}(x, y) \\
=\int_{-\infty}^{\infty}\left(\int_{-\infty}^{\infty}\left|\mathrm{e}^{-i x \xi} f(x-y) g(y)\right| \mathrm{d} x\right) \mathrm{d} y \\
=\int_{-\infty}^{\infty}|g(y)|\left(\int_{-\infty}^{\infty}\left|\mathrm{e}^{-i x \xi} f(x-y)\right| \mathrm{d} x\right) \mathrm{d} y=\|f\|_{1}\|g\|_{1}<\infty . \\
\therefore \widehat{f * g}(\xi)=\int_{-\infty}^{\infty} g(y)\left(\int_{-\infty}^{\infty} \mathrm{e}^{-i x \xi} f(x-y) \mathrm{d} x\right) \mathrm{d} y \\
=\int_{-\infty}^{\infty} g(y) \mathcal{F}_{x}(f(x-y))(\xi) \mathrm{d} y=\int_{-\infty}^{\infty} g(y) \mathrm{e}^{-i \xi y} \hat{f}(\xi) \mathrm{d} y \\
=\hat{f}(\xi) \hat{g}(\xi) .
\end{gathered}
$$




\subsubsection{Funções de Schwartz}

Definição 3.12. (i) Dizemos que uma função $f: \mathbb{R} \rightarrow \mathbb{C}$ é de decrescimento rápido se

$$
\lim _{|x| \rightarrow \infty} x^{n} f(x)=0, \quad \forall n \in \mathbb{N} .
$$

(ii) Uma função de Schwartz em $\mathbb{R}$ é uma função $\mathcal{C}^{\infty} f: \mathbb{R} \rightarrow \mathbb{C}$ tal que as derivadas $f^{(n)}$ são de decrescimento rápido para todo $n \in \mathbb{N}$.

Denotamos por $\mathcal{S}(\mathbb{R})$ o conjunto de todas as funções de Schwartz em $\mathbb{R}$.

Observação 3.13 (Funções de múltiplas variáveis). Para que uma função $f$ : $\mathbb{R}^{N} \rightarrow \mathbb{C}$ seja de Schwartz, pede-se que

$$
\lim _{\|x\| \rightarrow \infty}\|x\|^{n} D^{\alpha} f(x)=0
$$

para todos $n \in \mathbb{N}$ e $\alpha \in \mathbb{N}^{N}$, onde $D^{\alpha}=\partial_{1}^{\alpha_{1}} \cdots \partial_{N}^{\alpha_{N}}$ e $\|$.$\| é uma norma$ pré-fixada em $\mathbb{R}^{N}$.

Proposição 3.14. $\quad$ (i) $\mathcal{S}(\mathbb{R})$ é um espaço vetorial.

(ii) Se $f \in \mathcal{S}(\mathbb{R}), f^{\prime} \in \mathcal{S}(\mathbb{R})$.

(iii) Se $P(x)$ é um polinômio em $x$ e $f \in \mathcal{S}(\mathbb{R}), x \mapsto P(x) f(x) \in \mathcal{S}(\mathbb{R})$.

(iv) Se $f \in \mathcal{S}(\mathbb{R})$, f é limitada.

(v) $\mathcal{S}(\mathbb{R}) \subset L^{1}(\mathbb{R})$

Demonstração. Mostremos que $\mathcal{S}(\mathbb{R})$ é um espaço vetorial.Sejam $f, g \in \mathcal{S}(\mathcal{R})$ e $\lambda \in \mathbb{C}, n, m \in \mathbb{N}$.

$$
\lim _{|x| \rightarrow \infty} x^{m}(f+\lambda g)^{(n)}(x)=\lim _{|x| \rightarrow \infty} x^{m} f^{(n)}(x)+\lambda \lim _{|x| \rightarrow \infty} x^{m} g^{(n)}(x)=0 .
$$

$\operatorname{Assim} f+\lambda g \in \mathcal{S}(\mathbb{R})$.

Mostremos que toda $f \in \mathcal{S}(\mathbb{R})$ tem $f^{\prime} \in \mathcal{S}(\mathbb{R})$.

$$
\lim _{|x| \rightarrow \infty} x^{m}\left(f^{\prime}\right)^{(n)}(x)=\lim _{|x| \rightarrow \infty} x^{m} f^{(n+1)}(x)=0 .
$$

Assim, $f^{\prime} \in \mathcal{S}(\mathbb{R})$. 
Mostremos que, para toda $f \in \mathcal{S}(\mathbb{R})$ e para todo polinômio $P(x), x \mapsto$ $P(x) f(x) \in \mathcal{S}(\mathbb{R})$. Seja $P(x)=\sum_{j=0}^{p} a_{j} x^{j}$. Então

$$
\begin{aligned}
\lim _{|x| \rightarrow \infty} x^{m} P(x) f(x)= & \lim _{|x| \rightarrow \infty} x^{m}\left(\sum_{j=0}^{p} a_{j} x^{j}\right) f(x) \\
& =\lim _{|x| \rightarrow \infty} \sum_{j=0}^{p} a_{j} x^{j+m} f(x)=\sum_{j=0}^{p} a_{j} \lim _{|x| \rightarrow \infty} x^{j+m} f(x)=0
\end{aligned}
$$

Assim, $x \mapsto P(x) f(x)$ é de decrescimento rápido. Como todas as derivadas de $P(x) f(x)$ são somas da forma $P_{1}(x) f_{1}(x)+\cdots+P_{k}(x) f_{k}(x)$, onde $P_{1}, \ldots, P_{k}$ são polinômios e $f_{1}, \ldots, f_{k}$ são funções de Schwartz, teremos o resultado desejado.

Mostremos que toda $f \in \mathcal{S}(\mathbb{R})$ é limitada. Como $\lim _{|x| \rightarrow \infty} f(x)=0$, existe $K>0$ tal que $|x|>K \Rightarrow|f(x)|<1$. Como $f$ é contínua, $f$ é limitada em $[-K, K]$. Assim, $|f(x)| \leq \max \left\{\sup _{K}|f|, 1\right\}, \forall x \in \mathbb{R}$.

Mostremos que toda $f \in \mathcal{S}(\mathbb{R})$ é integrável. $x \mapsto\left(1+x^{2}\right) f(x)$ pertence a $\mathcal{S}(\mathbb{R}), \operatorname{assim} x \mapsto\left(1+x^{2}\right) f(x)$ é limitada por algum $L>0$.

$$
\left|\left(1+x^{2}\right) f(x)\right| \leq L \therefore|f(x)| \leq \frac{L}{1+x^{2}}, \forall x \in \mathbb{R} .
$$

Assim,

$$
\int_{-\infty}^{\infty}|f(x)| \mathrm{d} x \leq L \int_{-\infty}^{\infty} \frac{1}{1+x^{2}} \mathrm{~d} x=L \pi \therefore f \in L^{1}(\mathbb{R})
$$

Proposição 3.15. Se $f \in \mathcal{S}(\mathbb{R})$, então $\hat{f} \in \mathcal{S}(\mathbb{R})$.

Demonstração. Primeiro, mostremos que $\hat{f} \in \mathcal{C}^{\infty}(\mathbb{R})$.

$$
\begin{gathered}
x \mapsto x f(x) \in L^{1}(\mathbb{R}) \Rightarrow \exists(\hat{f})^{\prime}=\mathcal{F}_{x}(-i x f(x)) . \\
x \mapsto-i x^{2} f(x) \in L^{1}(\mathbb{R}) \Rightarrow \exists(\hat{f})^{\prime \prime}=\mathcal{F}_{x}(-i x f(x))^{\prime}=\mathcal{F}_{x}\left((-i)^{2} x^{2} f(x)\right) . \\
\vdots \\
\left.x \mapsto(-i)^{k-1} x^{k} f(x)\right) \in L^{1}(\mathbb{R}) \Rightarrow \exists(\hat{f})^{(k)}=\mathcal{F}_{x}\left((-1)^{k} x^{k} f(x)\right) .
\end{gathered}
$$


Em seguida, mostremos que $\hat{f}$ tem decrescimento rápido.

$$
\begin{aligned}
\lim _{|\xi| \rightarrow \infty} \xi \hat{f}(\xi) & =\lim _{|\xi| \rightarrow \infty} \frac{1}{i}\left(f^{\prime}\right)^{\wedge}(\xi)=0 . \\
\lim _{|\xi| \rightarrow \infty} \xi^{2} \hat{f}(\xi) & =\lim _{|\xi| \rightarrow \infty}\left(\frac{1}{i}\right)^{2}\left(f^{\prime \prime}\right)^{\wedge}(\xi)=0 . \\
\vdots & \\
\lim _{|\xi| \rightarrow \infty} \xi^{k} \hat{f}(\xi) & =\lim _{|\xi| \rightarrow \infty}\left(\frac{1}{i}\right)^{k}\left(f^{(k)}\right)^{\wedge}(\xi)=0 .
\end{aligned}
$$

Já mostramos que a transformada de Fourier de uma função de Schwartz é sempre suave e tem decrescimento rápido. Falta mostrar que as derivadas de $\hat{f}$ têm decrescimento rápido. Mas

$$
(\hat{f})^{(n)}=\mathcal{F}_{x}\left((-i x)^{n} f(x)\right),
$$

que é a uma transformada de Fourier de função de Schwartz e, portanto, tem decrescimento rápido.

Exemplo 3.16. Seja $g: \mathbb{R} \rightarrow \mathbb{R}$ a função gaussiana.

$$
g(x)=\mathrm{e}^{-x^{2}} .
$$

É fácil ver que $g$ tem decrescimento rápido:

$$
\lim _{|x| \rightarrow \infty} x^{n} \mathrm{e}^{-x^{2}}=\lim _{|x| \rightarrow \infty} \frac{x^{n}}{\mathrm{e}^{x^{2}}}=0 \quad \text { (Regra de L'Hospital). }
$$

Assim, $x \mapsto P(x) g(x)$ também tem decrescimento rápido, onde $P$ é um polinômio qualquer. Como todas as derivadas de $g$ são da forma $x \mapsto P(x) g(x)$, $g$ é uma função de Schwartz.

Exemplo 3.17 (Autovetor da transformada de Fourier). Considere $g$ a função gaussiana. Vimos no exemplo 3.7 que $\hat{g}(\xi)=\sqrt{\pi} g(\xi / 2)$. Fazendo $\lambda=1 / \sqrt{2}$, defina

$$
\begin{gathered}
f(x)=\mathrm{e}^{-\frac{x^{2}}{2}}=\mathrm{e}^{-\lambda^{2} x^{2}}=g(\lambda x) \\
\therefore \hat{f}(\xi)=\frac{1}{\lambda} \hat{g}\left(\frac{\xi}{\lambda}\right)=\frac{\sqrt{\pi}}{\lambda} g\left(\frac{\xi}{2 \lambda}\right)=\frac{\sqrt{\pi}}{\lambda} f\left(\frac{\xi}{2 \lambda^{2}}\right)=\sqrt{2 \pi} f(\xi) .
\end{gathered}
$$

Assim, temos que $f(x)=\mathrm{e}^{-\frac{x^{2}}{2}}$ é um autovetor da transformada de Fourier em $\mathcal{S}(\mathbb{R})$ e seu autovalor associado é $\sqrt{2 \pi}$. 


\subsubsection{Transformada de Fourier inversa}

Definição 3.18. Dada $f \in L^{1}(\mathbb{R})$, denotaremos por $\check{f}$ ou $\mathcal{F}_{\xi}^{-1}(f(\xi))$ a seguinte função, chamada de transformada de Fourier inversa de $f$ :

$$
\check{f}(x)=\frac{1}{2 \pi} \int_{-\infty}^{\infty} \mathrm{e}^{i \xi x} f(\xi) \mathrm{d} \xi .
$$

Note que $\check{f}=\frac{1}{2 \pi} \hat{\tilde{f}}$.

Observação 3.19. Vimos, nas propriedades da transformada de Fourier, que $\tilde{\hat{f}}=\hat{\tilde{f}}$. O mesmo vale para a transformada inversa: $\tilde{\tilde{f}}=\tilde{\tilde{f}}$.

Lema 3.20. Sejam $f, g \in L^{1}(\mathbb{R})$. Então

$$
\int \hat{f} g=\int f \hat{g}
$$

Demonstração.

$$
\begin{aligned}
& \int_{-\infty}^{\infty} \hat{f}(x) g(x) \mathrm{d} x=\int_{-\infty}^{\infty}\left(\int_{-\infty}^{\infty} \mathrm{e}^{-i x y} f(y) \mathrm{d} y\right) g(x) \mathrm{d} x \\
&=\iint_{\mathbb{R}^{2}} \mathrm{e}^{-i x y} f(y) g(x) \mathrm{d} y \mathrm{~d} x=\int_{-\infty}^{\infty}\left(\int_{-\infty}^{\infty} \mathrm{e}^{-i x y} g(x) \mathrm{d} x\right) f(y) \mathrm{d} y \\
& \quad=\int_{-\infty}^{\infty} f(y) \hat{g}(y) \mathrm{d} y .
\end{aligned}
$$

Proposição 3.21. Seja $f \in L^{1}(\mathbb{R})$ tal que $\hat{f} \in L^{1}(\mathbb{R})$. Então

$$
\hat{\hat{f}}(x)=2 \pi f(-x)
$$

para quase todo $x$.

Demonstração. Seja $G(x)=\mathrm{e}^{-x^{2}}$.

$$
\begin{gathered}
\hat{\hat{f}}(x)=\int_{-\infty}^{\infty} \mathrm{e}^{-i x y} \hat{f}(y) \mathrm{d} y=\lim _{M \rightarrow \infty} \int_{-\infty}^{\infty} G\left(\frac{1}{M} y\right) \mathrm{e}^{-i x y} \hat{f}(y) \mathrm{d} y \\
\int_{-\infty}^{\infty} G\left(\frac{1}{M} y\right) \mathrm{e}^{-i x y} \hat{f}(y) \mathrm{d} y=\int_{-\infty}^{\infty} G\left(\frac{1}{M} y\right) \mathcal{F}_{u}(f(u-x))(y) \mathrm{d} y \\
=\int_{-\infty}^{\infty} \mathcal{F}_{t}\left(G\left(\frac{1}{M} t\right)\right)(y) f(y-x) \mathrm{d} y \\
=\int_{-\infty}^{\infty} M \widehat{G}(M y) f(y-x) \mathrm{d} y=\int_{-\infty}^{\infty} \widehat{G}_{M}(y) f(x-y) \mathrm{d} y
\end{gathered}
$$


$\widehat{G}$ tem majorante radial integrável igual a ele mesmo e $\int \widehat{G}=(G)^{\hat{\wedge}}(0)=$ $2 \pi G(0)=2 \pi$ (ver exemplo 3.7). Assim, pelo corolário 3.3,

$$
\lim _{M \rightarrow \infty} \int_{-\infty}^{\infty} \widehat{G}_{M}(y) f(y-x) \mathrm{d} y=2 \pi f(-x),
$$

para quase todo $x \in \mathbb{R}$.

Corolário 3.22. Suponha que $f$ e $\hat{f}$ ambas pertencem a $L^{1}(\mathbb{R})$ (e, portanto, também $\check{f}$ ). Então

$$
\begin{gathered}
(\check{f})^{\wedge}=\frac{1}{2 \pi}(\tilde{f})^{\wedge}=\tilde{\tilde{f}}=f \quad e \\
(\hat{f})^{\curvearrowright}=\frac{1}{2 \pi}(\hat{f})^{\hat{\imath}}=\frac{1}{2 \pi} \hat{\tilde{\tilde{f}}}=\tilde{\tilde{f}}=f,
\end{gathered}
$$

onde as igualdades são em quase todo ponto.

A seguinte equação, válida para quase todo $x$, é chamada de Fórmula Inversa de Fourier:

$$
f(x)=\frac{1}{2 \pi} \int_{-\infty}^{\infty} \mathrm{e}^{i \xi x} \hat{f}(\xi) \mathrm{d} \xi .
$$

Corolário 3.23. Sejam $f, g \in \mathcal{S}(\mathbb{R})$. Então $f g \in \mathcal{S}(\mathbb{R}) e$

$$
\widehat{f g}=\frac{1}{2 \pi} \hat{f} * \hat{g} .
$$

Demonstração. Para ver que $f g \in \mathcal{S}(\mathbb{R})$, basta notar que

$$
x^{n}(f g)^{(m)}(x)=x^{n} \sum_{j=1}^{m}\left(\begin{array}{c}
m \\
j
\end{array}\right) f^{(j)}(x) g^{(m-j)}(x)
$$

se anula quando $|x| \rightarrow \infty$ para quaisquer $m, n$ naturais.

$$
\begin{gathered}
f g=(\check{f} * \check{g})^{\wedge} \\
\therefore \widehat{f g}=(\check{f} * \check{g})^{\wedge}=2 \pi(\check{f} * \check{g})^{\sim}=2 \pi(\tilde{f} * \tilde{g})=\frac{1}{2 \pi}(\hat{f} * \hat{g}) .
\end{gathered}
$$

Proposição 3.24 (Teorema de Plancherel). Seja $f \in L^{2}(\mathbb{R}) \cap \mathcal{S}(\mathbb{R})$. Então

$$
\|\hat{f}\|_{2}=\sqrt{2 \pi}\|f\|_{2} .
$$


Demonstração. Para começar, note que

$$
\overline{(\hat{f})}(\xi)=\int_{-\infty}^{\infty} \overline{\mathrm{e}^{-i \xi y} f(x)} \mathrm{d} x=\int_{-\infty}^{\infty} \mathrm{e}^{i \xi y} \overline{f(x)} \mathrm{d} x=\widehat{(\bar{f})}(-\xi)=\tilde{\hat{f}}(\xi) .
$$

Note também que o operador ${ }^{\sim}$ comuta com a transformada de Fourier e com a conjugação.

$$
\begin{array}{r}
\|\hat{f}\|_{2}^{2}=\int_{-\infty}^{\infty} \hat{f}(y) \overline{\hat{f}(y)} \mathrm{d} y=\int_{-\infty}^{\infty} \hat{f}(y) \widehat{\widehat{\tilde{f}(y)}} \mathrm{d} y=\int_{-\infty}^{\infty} f(y) \hat{\widehat{\hat{\tilde{f}}(y)}} \mathrm{d} y \\
=2 \pi \int_{-\infty}^{\infty} f(y) \overline{f(y)} \mathrm{d} y=2 \pi\|f\|_{2}^{2} .
\end{array}
$$

\subsubsection{Aplicação: o núcleo de Poisson no semiplano}

Observação 3.25. Vamos procurar soluções da seguinte equação

$$
\frac{\partial^{2} u}{\partial x^{2}}+\frac{\partial^{2} u}{\partial y^{2}}=0
$$

definidas no semiplano $y>0$ de $\mathbb{R}^{2}$ e que se anulam no infinito. Supondo que $u$ é suficientemente bem-comportada para isto, aplicamos a transformada de Fourier em relação à variável $x$ para obter

$$
-\xi^{2} \hat{u}(\xi, y)+\frac{\partial^{2} \hat{u}(\xi, y)}{\partial y^{2}}=0 .
$$

As soluções desta E.D.O. em y que nos interessam (por não crescerem demasiadamente no infinito) são dadas por

$$
\hat{u}(\xi, y)=k(\xi) \mathrm{e}^{-|\xi| y},
$$

Como a transformada de Fourier transforma convoluções em produtos, a solução $u$ será da forma

$$
u(x, y)=\check{k}(x) * \mathcal{F}_{\xi}^{-1}\left(\mathrm{e}^{-|\xi| y}\right)(x),
$$

onde o asterisco denota convolução em relação à variável $x$.

Calculemos a transformada inversa de $\mathrm{e}^{-|\xi| y}$, que será chamada de núcleo de Poisson no semiplano.

$$
\mathcal{F}_{\xi}^{-1}\left(\mathrm{e}^{-|\xi| y}\right)(x)=\frac{1}{y} \mathcal{F}_{\xi}^{-1}\left(\mathrm{e}^{-|\xi|}\right)\left(\frac{x}{y}\right) .
$$




$$
\mathcal{F}_{\xi}^{-1}\left(\mathrm{e}^{-|\xi|}\right)(x)=\frac{1}{2 \pi} \int_{-\infty}^{\infty} \cos (x \xi) \mathrm{e}^{-|\xi|} \mathrm{d} \xi=\frac{1}{\pi} \int_{0}^{\infty} \cos (x \xi) \mathrm{e}^{-\xi} \mathrm{d} \xi .
$$

Para integrar $\cos (x \xi) \mathrm{e}^{-\xi}$, podemos fazer a integração por partes duas vezes:

$$
\begin{gathered}
I=\int_{0}^{\infty} \cos (x \xi) \mathrm{e}^{-\xi}=-\left.\mathrm{e}^{-\xi} \cos (x \xi)\right|_{0} ^{\infty}-\int_{0}^{\infty}(-x \operatorname{sen}(x \xi))\left(-\mathrm{e}^{-\xi}\right) \mathrm{d} \xi \\
=1-x \int_{0}^{\infty} \operatorname{sen}(x \xi) \mathrm{e}^{-\xi} \mathrm{d} \xi \\
\int_{0}^{\infty} \operatorname{sen}(x \xi) \mathrm{e}^{-\xi} \mathrm{d} \xi=-\left.\mathrm{e}^{-\xi} \operatorname{sen}(x \xi)\right|_{0} ^{\infty}-\int_{0}^{\infty} x \cos (x \xi)\left(-\mathrm{e}^{-\xi}\right) \mathrm{d} \xi \\
=x \int_{0}^{\infty} \cos (x \xi) \mathrm{e}^{-\xi} \mathrm{d} \xi=x I \\
\therefore I=1-x^{2} I \quad \therefore I\left(1+x^{2}\right)=1 \quad \therefore I=\frac{1}{1+x^{2}} .
\end{gathered}
$$

Assim, temos

$$
u(x, y)=\check{k}(x) * P_{y}(x),
$$

onde estamos usando a seguinte notação:

$$
P(x)=\frac{1}{\pi} \frac{1}{1+x^{2}}, \quad P_{y}(x)=\frac{1}{y} P\left(\frac{x}{y}\right)=\frac{1}{\pi} \frac{y}{x^{2}+y^{2}} .
$$

Como $P$ tem majorante radial integrável e integral unitária, o corolário 3.3 nos diz que

$$
\lim _{y \rightarrow 0} \check{k}(x) * P_{y}(x)=\check{k}(x)
$$

para quase todo $x$. A solução da equação fica sendo determinada pelo valor limite de $u$ no eixo $x$, ou seja:

$$
u(x, y)=u\left(x, 0^{+}\right) * P_{y}(x) .
$$




\section{Capítulo 4}

\section{Sobre a Condição $(\mathcal{P})$ em $\mathbb{R}^{2}$}

\subsection{Resolubilidade Local}

Definição 4.1. Seja $L$ um campo vetorial (complexo) definido em um aberto $\Omega \subset \mathbb{R}^{2}$ e seja $p \in \Omega$. Dizemos que $L$ é localmente resolúvel em $p$ se existe uma vizinhança $U=U(p)$ tal que, para toda $f \in \mathcal{C}^{\infty}(\Omega)$, existe $u \in \mathcal{D}^{\prime}(\Omega)$ tal que $L u=f$ em $U$ (isto é, $L u(\phi)=\int_{\Omega} f(x) \phi(x) \mathrm{d} x$ para toda função teste $\phi$ com suporte contido em $U$ ).

Observação 4.2. Dado $\Omega \subset \mathbb{R}^{N}$ um aberto, estamos denotando por $\mathcal{D}^{\prime}(\Omega)$ o espaço das distribuições (no sentido de Laurent Schwartz) em $\Omega$, isto é, o dual do espaço $\mathcal{C}_{c}^{\infty}(\Omega)$ das funções suaves em $\Omega$ com suporte compacto (que pode ser identificado com o espaço das funções suaves em $\mathbb{R}^{N}$ com suporte compacto contido em $\Omega$ ), dotado da topologia apropriada. Para uma introdução à teoria das distribuições, ver Hormänder [2].

Observação 4.3. A definição será equivalente se supormos que as distribuições $u$ e as funções $f$ têm suporte compacto.

Observação 4.4. Equivalentemente, podemos pedir nesta definição que exista $u \in \mathcal{D}^{\prime}(U)$ tal que $L u=\left.f\right|_{U}$

Proposição 4.5. Sejam $\Omega \subset \mathbb{R}^{2}$ aberto, $(x, t)=\left.\mathrm{Id}\right|_{\Omega}$ as coordenadas usuais $d e \Omega e$

$$
L=A \frac{\partial}{\partial t}+B \frac{\partial}{\partial x}
$$

um campo vetorial complexo em $\Omega$, com $A, B \in \mathcal{C}^{\infty}(\Omega)$. Vamos supor que $|A|+|B|>0$ em $\Omega$. Dado $p \in \Omega,|A|$ ou $|B|$ é diferente de zero em alguma vizinhança $\Omega_{p}$ de $p$. Vamos supor que este campo é $A$. Então definiremos em $\Omega_{p}$ o campo $\tilde{L}$ dado por $(1 / A) L$, isto é,

$$
\tilde{L}=\left.\frac{\partial}{\partial t}\right|_{\Omega_{p}}+\left.\tilde{B} \frac{\partial}{\partial x}\right|_{\Omega_{p}},
$$


onde

$$
\tilde{B}=\frac{\left.B\right|_{\Omega_{p}}}{\left.A\right|_{\Omega_{p}}} \in \mathcal{C}^{\infty}\left(\Omega_{p}\right) .
$$

$L$ é localmente resolúvel em $p$ se e somente $\tilde{L}$ o for.

Demonstração. Suponha que $L$ é localmente resolúvel em $p$. Seja $U_{p}$ uma vizinhança de $p$ tal que, para toda $f \in \mathcal{C}^{\infty}(\Omega)$, existe $u \in \mathcal{D}^{\prime}(\Omega)$ tal que $L u=f$ em $U_{p}$.

Seja $U_{p}^{\prime}$ uma vizinhança de $p$ tal que $U_{p}^{\prime} \subset \subset U_{p} \cap \Omega_{p}$. Dada $f \in \mathcal{C}^{\infty}\left(\Omega_{p}\right)$, seja $\tilde{f} \in \mathcal{C}^{\infty}(\Omega)$ que coincide com $f$ em $U_{p}^{\prime}$. Então existe $u \in \mathcal{D}^{\prime}(\Omega)$ tal que

$$
L u=A \tilde{f} \text { em } U_{p} .
$$

Seja $\tilde{u}=\left.u\right|_{\Omega_{p}}$. Então

$$
\left.L\right|_{\Omega_{p}} \tilde{u}=\left.A\right|_{\Omega_{p}} f \text { em } U_{p}^{\prime}
$$

Portanto, como $\tilde{L}=\left.\left(1 /\left.A\right|_{\Omega_{p}}\right) L\right|_{\Omega_{p}}$,

$$
\tilde{L} \tilde{u}=f \operatorname{em} U_{p}^{\prime}
$$

Temos que a resolubilidade local de $L$ em $p$ implica a resolubilidade local de $\tilde{L}$ em $p$.

Por outro lado, suponha que $\tilde{L}$ é localmente resolúvel em $p$. Seja $V_{p}$ a vizinhança de $p$ associada. Podemos tomar uma $V_{p} \subset \subset \Omega_{p}$. Dada $g \in \mathcal{C}^{\infty}(\Omega)$, tome $u \in \mathcal{D}^{\prime}\left(\Omega_{p}\right)$ tal que

$$
\tilde{L} u=\left.\frac{1}{\left.A\right|_{\Omega_{p}}} g\right|_{\Omega_{p}} \text { em } V_{p} .
$$

Afirmação: existe uma distribuição $u_{0} \in \mathcal{D}^{\prime}(\Omega)$ tal que $u=u_{0}$ em $V_{p}$. De fato, tome $\psi \in \mathcal{C}_{c}^{\infty}(\Omega)$ tal que $\operatorname{supp}(\psi) \subset \Omega_{p}$ e que vale $1 \mathrm{em} V_{p}$. Então $u_{0}(\phi):=u\left(\left.(\psi \phi)\right|_{\Omega_{p}}\right)$ é a distribuição desejada.

Portanto,

$$
L u_{0}=g \text { em } V_{p} .
$$

Proposição 4.6. Seja

$$
L=\frac{\partial}{\partial t}+B \frac{\partial}{\partial x}
$$


um campo vetorial complexo definido em um aberto $\Omega$ de $\mathbb{R}^{2}$, onde $(x, t)=$ $\left.\mathrm{Id}\right|_{\Omega}$ são as coordenadas usuais e $B \in \mathcal{C}^{\infty}(\Omega)$. Então existe uma carta local $(\xi, s): U \subset \Omega \rightarrow V \subset \mathbb{R}^{2}$, com $\xi=x, p \in U$, tal que

$$
\left.L\right|_{U}=\frac{\partial}{\partial s}+i b \frac{\partial}{\partial \xi}
$$

onde $b \in \mathcal{C}^{\infty}(U)$ assume somente valores reais.

Demonstração. Escrevamos $B=B_{1}+i B_{2}$, onde $B_{1}$ é a parte real de $B$ e $B_{2}$ é a parte imaginária de $B$. Então

$$
L=\frac{\partial}{\partial t}+B_{1} \frac{\partial}{\partial x}+i B_{2} \frac{\partial}{\partial x} .
$$

Sejam $Y=\partial_{t}+B_{1} \partial_{x}, p=\left(x_{0}, t_{0}\right)$. A aplicação

$$
\phi:\left(\xi^{\prime}, s^{\prime}\right) \longmapsto Y_{s^{\prime}}\left(x_{0}+\xi^{\prime}, t_{0}\right)
$$

(que é a aplicação $\rho_{\eta, p} \operatorname{com} \eta=\left(Y, \partial_{x}\right)$ conforme definida na subseção 2.3.4) tem posto dois em $p$ e é suave, portanto é localmente invertível. Seja $(\xi, s)$ o seu inverso local (também suave) em torno de $p$, ou seja, $(\xi, s)$ é uma carta local que satisfaz (onde é definida)

$$
(x, t)=Y_{s}\left(x_{0}+\xi, t_{0}\right) .
$$

Pela proposição 1.13,

$$
\frac{\partial}{\partial \xi}=\frac{\partial x}{\partial \xi} \frac{\partial}{\partial x}+\frac{\partial t}{\partial \xi} \frac{\partial}{\partial t} \quad \text { e } \quad \frac{\partial}{\partial s}=\frac{\partial x}{\partial s} \frac{\partial}{\partial x}+\frac{\partial t}{\partial s} \frac{\partial}{\partial t} .
$$

Com a variação do parâmetro $s$ (e mantendo $\xi$ constante), $(x, t)$ é uma curva integral de $Y$. Com isto, obtemos

$$
\frac{\partial}{\partial s}=Y=\frac{\partial}{\partial t}+B_{1} \frac{\partial}{\partial x} .
$$

Repare que

$$
\begin{gathered}
t=t\left(Y_{s}\left(x_{0}+\xi, t_{0}\right)\right)=t_{0}+s, \\
\therefore \frac{\partial t}{\partial \xi}=\frac{\partial}{\partial \xi}\left(t_{0}+s\right)=0 .
\end{gathered}
$$

Assim,

$$
\frac{\partial}{\partial \xi}=\frac{\partial x}{\partial \xi} \frac{\partial}{\partial x}
$$


Portanto, fazendo

$$
b=\frac{1}{\left(\frac{\partial x}{\partial \xi}\right)} B_{2}
$$

temos o resultado desejado.

Exemplo 4.7 (Caso "tubo"). Vamos supor que $b$ não depende de $x$. Ou seja, $L=\partial_{t}+i b(t) \partial_{x}$ com $b \in \mathcal{C}^{\infty}(\mathbb{R})$, onde $b$ assume somente valores reais. Vamos procurar condições suficientes para que $L$ seja localmente resolúvel na origem.

Consideremos a equação

$$
\frac{\partial u}{\partial t}+i b(t) \frac{\partial u}{\partial x}=f
$$

Vamos supor que $u$ é uma função e que podemos aplicar a transformada de Fourier parcial da equação em relação à variável $x$. Temos

$$
\frac{\mathrm{d} \hat{u}}{\mathrm{~d} t}(\xi, t)-b(t) \xi \hat{u}(\xi, t)=\hat{f}(\xi, t) .
$$

Resolvendo esta equação diferencial, obtemos

$$
\hat{u}(\xi, t)=\int_{T}^{t} \mathrm{e}^{\xi(B(t)-B(s))} \hat{f}(\xi, s) \mathrm{d} s,
$$

onde $B$ é uma antiderivada de $b$ e $T$ é um número real. Para poder fazer a transformada de Fourier inversa de $\hat{u}(\xi, t)$ em relação a $\xi$, temos que controlar o seu crescimento em relação à variável $\xi$.

Se $\hat{f}(\xi, t)=0$ sempre que $|\xi|>1$ : neste caso, $\hat{u}(\xi, t)$ será uma função de Schwartz em relação a $\xi$. Aplicando a fórmula da transformada inversa de Fourier (e usando $T(\xi)=0$ ), obtemos uma solução

$$
u(x, t)=\frac{1}{2 \pi} \int_{-\infty}^{\infty} \int_{0}^{t} \mathrm{e}^{i \xi x+\xi(B(t)-B(s))} \hat{f}(\xi, s) \mathrm{d} s \mathrm{~d} \xi
$$

que funciona em todo o plano.

Se $\hat{f}(\xi, t)=0$ sempre que $\xi<0$ : para que $\hat{u}(\xi, t)$ seja uma função de Schwartz em relação a $\xi$, devemos exigir que

$$
B(t)-B(s) \leq 0, \text { sempre que } s \text { está entre } T \text { e } t .
$$

Pelo teorema do valor médio, $B(t)-B(s)=b(\eta)(t-s)$, onde $\eta$ está entre $t$ e $s$. 
Vamos supor que $b$ não muda de sinal na origem. Isto é, existe $\delta>0$ tal que, para todo $t \in[-\delta, \delta], b(t) \geq 0$ ou para todo $t \in[-\delta, \delta], b(t) \leq 0$. Caso $b(t) \geq 0$ em $(-\delta, \delta)$, faça $T=-\delta$. Caso $b(t) \leq 0 \mathrm{em}(-\delta, \delta)$, faça $T=\delta$. Assim, $\hat{u}(\xi, t)$ será uma função de Schwartz em relação a $\xi$ sempre que $|t|<\delta$. Aplicando novamente a fórmula inversa de Fourier, temos uma solução local,

$$
u(x, t)=\frac{1}{2 \pi} \int_{-\infty}^{\infty} \int_{T}^{t} \mathrm{e}^{i \xi x+\xi(B(t)-B(s))} \hat{f}(\xi, s) \mathrm{d} s \mathrm{~d} \xi,
$$

definida em $\mathbb{R} \times(-\delta, \delta)$.

Se $\hat{f}(\xi, t)=0$ sempre que $\xi>0$ : podemos encontrar uma solução local de $L u=f$ em $\mathbb{R} \times(-\delta, \delta)$, de forma análoga — onde o $\delta$ é tal que $b$ não muda de sinal em $(-\delta, \delta)$.

Se $f$ é uma função suave em $\mathbb{R}^{2}$ que é de Schwartz em relação à coordenada $x$ : sejam

$$
\phi_{0}, \phi^{+}, \phi^{-}: \mathbb{R} \rightarrow[0,1]
$$

funções suaves, $\operatorname{com} \operatorname{supp} \phi_{0} \subset(-1,1), \operatorname{supp} \phi^{+} \subset(0, \infty)$ e $\operatorname{supp} \phi^{-} \subset$ $(-\infty, 0)$ tais que $1=\phi_{0}+\phi^{+}+\phi^{-}$.

Defina $f_{0}(x, t)=\mathcal{F}_{\xi}^{-1}\left(\hat{f}(\xi, t) \phi_{0}(\xi)\right)(x)$ e $f^{+}, f^{-}$analogamente. Então

$$
f=f_{0}+f^{+}+f^{-}
$$

$\widehat{f_{0}}(\xi, t)$ se anula quando $|\xi|>1, \widehat{f^{+}}(\xi, t)$ se anula quando $\xi<0$ e $\widehat{f^{-}}(\xi, t)$ se anula quando $\xi>0$. Assim, desde que $b$ não mude de sinal na origem, existem soluções $u^{-}, u^{+}$e $u_{0}$ tais que

$$
L u^{-}=f^{-}, \quad L u^{+}=f^{+} \quad \text { e } \quad L u_{0}=f_{0}
$$

em uma faixa $\mathbb{R} \times(-\delta, \delta)$, onde $\delta$ não depende de $f$. Fazendo $u=u_{0}+u^{+}+u^{-}$, temos que $L u=f$ em $\mathbb{R} \times(-\delta, \delta)$.

Caso geral: para $f \in \mathcal{C}^{\infty}\left(\mathbb{R}^{2}\right)$ qualquer, podemos pegar uma função $g$ que coincide com $f$ no retângulo $U=[0,1]^{2}$, mas que é de Schwartz em relação a $x$. Seja $u$ tal que $L u=g$ na faixa $\mathbb{R} \times(-\delta, \delta)$. Assim, $L u=f \mathrm{em}$ $U \cap \mathbb{R} \times(-\delta, \delta)$.

Concluímos que, para $L=\partial_{t}+i b(t) \partial_{x}$ ser localmente resolúvel na origem, é suficiente que $b$ não mude de sinal na origem.

\subsection{Uma primeira definição da condição $(\mathcal{P})$}

Definição 4.8. Seja $L$ um campo da forma

$$
\frac{\partial}{\partial t}+i b \frac{\partial}{\partial x}
$$


em $\mathbb{R}^{2}$, onde $b \in \mathcal{C}^{\infty}\left(\mathbb{R}^{2}\right)$ é uma função real. Dizemos que $L$ satisfaz a condição $(\mathcal{P})$ em $p=\left(x_{0}, t_{0}\right)$ se existe $\delta>0$ tal que, para todo $x \in\left(x_{0}-\right.$ $\left.\delta, x_{0}+\delta\right)$, a função $t \in\left(t_{0}-\delta, t_{0}+\delta\right) \mapsto b(x, t)$ não muda de sinal.

Observação 4.9. Vimos no "caso tubo" (exemplo 4.7) que a condição $(\mathcal{P})$, como definida acima, implica a resolubilidade local quando $b$ não depende de $x$. Veremos na seção 4.4 que isto também é verdade para qualquer $b$ suave, com solução fraca em $L^{2}$.

\subsection{Uma segunda definição da condição $(\mathcal{P})$}

\subsubsection{Parêntese: produto exterior de campos vetoriais}

Definição 4.10. Sejam $L_{1}, \ldots, L_{k} \in \mathfrak{X}(M)$ campos vetoriais na variedade $M$. Vamos definir o produto exterior $L_{1} \wedge \cdots \wedge L_{k}$ como sendo a aplicação multilinear e alternada

$$
L_{1} \wedge \cdots \wedge L_{k}:\left(\mathcal{C}^{\infty}(M)\right)^{k} \longrightarrow \mathcal{C}^{\infty}(M)
$$

definida por

$$
\begin{array}{r}
L_{1} \wedge \cdots \wedge L_{k}\left(f_{1}, \ldots, f_{k}\right)=\sum_{\sigma \in S^{k}} \operatorname{sgn}(\sigma) L_{1}\left(f_{\sigma(1)}\right) \cdots L_{k}\left(f_{\sigma(k)}\right) \\
=\operatorname{det}\left(\begin{array}{ccc}
L_{1}\left(f_{1}\right) & \ldots & L_{1}\left(f_{k}\right) \\
\vdots & \ddots & \vdots \\
L_{k}\left(f_{1}\right) & \ldots & L_{k}\left(f_{k}\right)
\end{array}\right) .
\end{array}
$$

Observação 4.11. Na notação " $L_{i}$ " usada aqui, $i$ é apenas o subíndice. Não iremos usar a notação $t \mapsto L_{t}$ para o fluxo do campo nesta seção.

Observação 4.12. Embora tenhamos feito a definição de produto exterior para campos vetoriais reais, a mesma também funcionaria para campos vetoriais complexos.

Observação 4.13 (Propriedades). Sejam $g \in \mathcal{C}^{\infty}(M), \sigma \in S^{k}, L_{1}, \ldots, L_{k} \in$ $\mathfrak{X}(M)$ e $L_{1}^{\prime} \in \mathfrak{X}(M)$. Então:

(i) $L_{\sigma(1)} \wedge \cdots \wedge L_{\sigma(k)}=\operatorname{sgn}(\sigma) L_{1} \wedge \cdots \wedge L_{k}$.

(ii) $\left(g L_{1}\right) \wedge L_{2} \wedge \cdots \wedge L_{k}=g\left(L_{1} \wedge L_{2} \wedge \cdots \wedge L_{k}\right)$.

(iii) $\left(L_{1}+L_{1}^{\prime}\right) \wedge L_{2} \wedge \cdots \wedge L_{k}=\left(L_{1} \wedge L_{2} \wedge \cdots \wedge L_{k}\right)+\left(L_{1}^{\prime} \wedge L_{2} \wedge \cdots \wedge L_{k}\right)$. 
Observação 4.14. Vamos supor que $\left(x_{1}, \ldots, x_{m}\right)$ é um sistema de coordenadas global para a variedade $M$. Aplicando a proposição 1.13 (expressão dos campos vetoriais em coordenadas locais) e as propriedades vistas acima, podemos calcular a fórmula de $L_{1} \wedge \cdots \wedge L_{k}$ em função das derivadas parciais $\partial_{x_{1}}, \ldots, \partial_{x_{m}}$ :

$$
\begin{aligned}
L_{1} \wedge \cdots \wedge L_{k}=\left(\sum_{i_{1}=1}^{m} L_{1}\left(x_{i_{1}}\right) \frac{\partial}{\partial x_{i_{1}}}\right) \wedge \cdots \wedge\left(\sum_{i_{k}=1}^{m} L_{k}\left(x_{i_{k}}\right) \frac{\partial}{\partial x_{i_{k}}}\right) \\
=\sum_{i_{1}, \ldots, i_{k}=1}^{m}\left(L_{1}\left(x_{i_{1}}\right) \frac{\partial}{\partial x_{i_{1}}}\right) \wedge \cdots \wedge\left(L_{k}\left(x_{i_{k}}\right) \frac{\partial}{\partial x_{i_{k}}}\right) \\
=\sum_{i_{1}, \ldots, i_{k}=1}^{m} L_{1}\left(x_{i_{1}}\right) \cdots L_{k}\left(x_{i_{k}}\right) \frac{\partial}{\partial x_{i_{1}}} \wedge \cdots \wedge \frac{\partial}{\partial x_{i_{k}}} .
\end{aligned}
$$

Como o produto $\partial_{x_{i_{1}}} \wedge \cdots \wedge \partial_{x_{i_{k}}}$ é nulo sempre que dois de $i_{1}, \ldots, i_{k}$ são iguais, a última soma pode ser reescrita como

$$
\begin{gathered}
\sum_{\substack{i_{1}, \ldots, i_{k}=1 \\
i_{1}<\cdots<i_{k}}}^{m} \sum_{\sigma \in S^{k}} L_{1}\left(x_{i_{\sigma(1)}}\right) \cdots L_{k}\left(x_{i_{\sigma(k)}}\right) \frac{\partial}{\partial x_{i_{\sigma(1)}}} \wedge \cdots \wedge \frac{\partial}{\partial x_{i_{\sigma(k)}}} \\
=\sum_{\substack{i_{1}, \ldots, i_{k}=1 \\
i_{1}<\cdots<i_{k}}}^{m} \sum_{\sigma \in S^{k}} \operatorname{sgn}(\sigma) L_{1}\left(x_{i_{\sigma(1)}}\right) \cdots L_{k}\left(x_{\left.i_{\sigma(k)}\right)}\right) \frac{\partial}{\partial x_{i_{1}}} \wedge \cdots \wedge \frac{\partial}{\partial x_{i_{k}}} \\
=\sum_{\substack{i_{1}, \ldots, i_{k}=1 \\
i_{1}<\cdots<i_{k}}}^{m} L_{1} \wedge \cdots \wedge L_{k}\left(x_{i_{1}}, \ldots, x_{i_{k}}\right) \frac{\partial}{\partial x_{i_{1}}} \wedge \cdots \wedge \frac{\partial}{\partial x_{i_{k}}} .
\end{gathered}
$$

Concluímos que

$$
L_{1} \wedge \cdots \wedge L_{k}=\sum_{\substack{i_{1}, \ldots, i_{k}=1 \\
i_{1}<\cdots<i_{k}}}^{m} \operatorname{det}\left(\begin{array}{ccc}
L_{1}\left(x_{i_{1}}\right) & \ldots & L_{1}\left(x_{i_{k}}\right) \\
\vdots & \ddots & \vdots \\
L_{k}\left(x_{i_{1}}\right) & \ldots & L_{k}\left(x_{i_{k}}\right)
\end{array}\right) \frac{\partial}{\partial x_{i_{1}}} \wedge \cdots \wedge \frac{\partial}{\partial x_{i_{k}}}
$$

\subsubsection{A definição em questão}

Definição 4.15. Seja $L=L_{1}+i L_{2}$ um campo vetorial complexo em um aberto $\Omega \subset \mathbb{R}^{2}$, com $L_{1}, L_{2}$ campos reais, tal que $L$ não se anula em nenhum ponto.

(i) Chamaremos as órbitas de $\left\{L_{1}, L_{2}\right\}$ de órbitas de $L$. 
(ii) Dizemos que $L$ satisfaz a condição $(\mathcal{P})$ em $\Omega$ se $L_{1} \wedge L_{2}$ não muda de sinal nas órbitas bidimensionais de $L$.

Observação 4.16. É necessário explicar o que queremos dizer quando pedimos que $L_{1} \wedge L_{2}$ não "mude de sinal" em uma órbita bidimensional de $L$. Seja $S$ uma órbita bidimensional de $L$. Como $S$ é uma subvariedade de dimensão maximal, $S$ é um aberto de $\Omega \subset \mathbb{R}^{2}$ e, portanto, é orientável. Sejam $(x, y)$ e $(z, w)$ duas cartas locais de $S$ que se interceptam e são da mesma orientação. Restrinja $L_{1}, L_{2}, x, y, z, w$ à intersecção de seus domínios.

$$
\frac{\partial}{\partial x} \wedge \frac{\partial}{\partial y}=\operatorname{det}\left(\begin{array}{ll}
\frac{\partial x}{\partial z} & \frac{\partial x}{\partial w} \\
\frac{\partial y}{\partial z} & \frac{\partial y}{\partial w}
\end{array}\right) \frac{\partial}{\partial z} \wedge \frac{\partial}{\partial w}=J \frac{\partial}{\partial z} \wedge \frac{\partial}{\partial w},
$$

onde $J$ é uma função estritamente positiva.

$$
L_{1} \wedge L_{2}=L_{1} \wedge L_{2}(x, y) \frac{\partial}{\partial x} \wedge \frac{\partial}{\partial y}=L_{1} \wedge L_{2}(x, y) J \frac{\partial}{\partial z} \wedge \frac{\partial}{\partial w}
$$

ou seja, $L_{1} \wedge L_{2}(z, w)=J L_{1} \wedge L_{2}(x, y)$, com $J>0$. Ambas terão o mesmo sinal.

Fixada uma orientação $\mathcal{O}$, definimos o sinal de $L_{1} \wedge L_{2}$ em cada ponto $p \in S$ como sendo o sinal de $L_{1} \wedge L_{2}\left(x^{\prime}, y^{\prime}\right)$, onde $\left(x^{\prime}, y^{\prime}\right)$ é uma carta local de orientação $\mathcal{O}$ contendo $p$.

Como $S$ é um aberto conexo de $\mathbb{R}^{2}$, só existem duas orientações possíveis - a positiva e a negativa. Trocar a orientação escolhida inverte o sinal de $L_{1} \wedge L_{2}$, o que não afeta a definição 4.15 .

Observação 4.17. Seja

$$
L=\frac{\partial}{\partial t}+i b \frac{\partial}{\partial x}
$$

definida em um aberto $\Omega \subset \mathbb{R}^{2}$, onde a função $b$ é real. Então as partes real e imaginária são $L_{1}=\partial_{t}$ e $L_{2}=b \partial_{x}$.

O produto exterior $L_{1} \wedge L_{2}=b \partial_{t} \wedge \partial_{x}$ tem o sinal de $-b$ (em relação à orientação de $(x, t))$.

As curvas integrais de $L_{1}$ são as retas verticais contidas em $\Omega$. As curvas integrais de $L_{2}$ são retas horizontais em $\Omega$ que não cruzam nenhum ponto em que $b=0$, ou então são constantes em um ponto em que $b=0$.

Se $b$ não muda de sinal nas órbitas de dimensão 2, tampouco mudará de sinal nas órbitas de dimensão 1 (que são retas verticais nas quais $b=0$ ). Nesse caso, o sinal de $b$ não mudará em nenhuma reta vertical contida em $\Omega$.

Por outro lado, seja $S$ uma órbita de dimensão 2, e sejam $p$ e $q$ dois pontos em $S$ tais que $b(p)>0$ e $b(q)<0$. Então $p$ e $q$ se ligam por um caminho feito de segmentos de curvas integrais de $L_{1}$ e $L_{2}$, e já sabemos que o sinal 
de $b$ não pode mudar nas curvas integrais de $L_{2}$. Concluímos que a mudança de sinal de $b$ ocorre em uma curva integral de $L_{1}$, que é uma reta vertical contida em $\Omega$.

Isto prova que, para este campo $L$, a condição $(\mathcal{P})$ da definição 4.15 é equivalente à condição $(\mathcal{P})$ da definição 4.8 valendo em todos os pontos de $\Omega$.

Observação 4.18. Seja $L$ um campo vetorial complexo não-singular definido no aberto $\Omega \subset \mathbb{R}^{2}$. Dado $p \in \Omega$, vimos (proposição 4.6) que existem coordenadas locais $(\xi, s)$ definidas em $U \subset \Omega$, com $p \in U$ nas quais

$$
\left.L\right|_{U}=\frac{\partial}{\partial s}+i b \frac{\partial}{\partial \xi}
$$

onde $b$ é uma função real. Se $p^{\prime} \in U$, a órbita de $\left.L\right|_{U}$ contendo $p^{\prime}$ está contida na órbita de $L$ contendo $p^{\prime}$. Portanto, se $L$ satisfaz a condição $(\mathcal{P})$ em $\Omega$, $\left.L\right|_{U}$ satisfaz a condição $(\mathcal{P})$ em $U$. Repetindo-se o raciocínio da observação anterior, isto implica que $b$ não muda de sinal nas curvas integrais de $\partial_{s}$.

\subsection{Resolubilidade local em $L^{2}$}

Teorema 4.19. Seja L um campo vetorial complexo em $\mathbb{R}^{2}$ da forma

$$
L=\frac{\partial}{\partial t}+i b \frac{\partial}{\partial x}
$$

com Id $\left.\right|_{\mathbb{R}^{2}}=(x, t)$, onde $b$ é uma função suave que só assume valores reais e tal que $L$ satisfaz a condição $(\mathcal{P})$ em uma vizinhança $U$ da origem. Então existem $a, T_{0}, C>0$ tais que, para todo $0<T \leq T_{0}$, dada $\phi \in \mathcal{C}_{c}^{\infty}((-a, a) \times$ $(-T, T))$,

$$
\|\phi\|_{L^{2}\left(\mathbb{R}^{2}\right)} \leq C T\left\|{ }^{t} L \phi\right\|_{L^{2}\left(\mathbb{R}^{2}\right)} .
$$

Demonstração. Vamos usar as letras $x$ e $t$ como variáveis, e não apenas como coordenadas locais. Podemos supor, sem perda de generalidade, que $b(x, t)$ tem suporte compacto e que a propriedade $(\mathcal{P})$ é valida em todo o plano.

${ }^{t} L$ é, por definição, o operador que satisfaz $\langle L \phi, \psi\rangle=\left\langle\phi,{ }^{t} L \psi\right\rangle$ para qualquer par de funções $\phi, \psi$ em $\mathcal{C}^{\infty}\left(\mathbb{R}^{2}\right)$, onde $\langle f, g\rangle:=\int f g$. Como

$$
\begin{aligned}
\langle L \phi, \psi\rangle & =\left\langle\phi_{t}, \psi\right\rangle+i\left\langle b \phi_{x}, \psi\right\rangle=-\left\langle\phi, \psi_{t}\right\rangle+i\left\langle\phi_{x}, b \psi\right\rangle \\
=-\left\langle\phi, \psi_{t}\right\rangle-i\left\langle\phi, b_{x} \psi\right\rangle-i\left\langle\phi b \psi_{x}\right\rangle=\left\langle\phi,-\psi_{t}-i b \psi_{x}-i b_{x} \psi\right\rangle & =\left\langle\phi,-L \phi-i b_{x} \phi\right\rangle
\end{aligned}
$$

então ${ }^{t} L=-L-i b_{x}$. 
Suponha que a estimativa do teorema vale para o campo $L$, ao invés de ${ }^{t} L$. Como $L=-i b_{x}-{ }^{t} L$,

$$
\begin{gathered}
\|\phi\|_{2} \leq C T\left\|i b_{x} \phi\right\|_{2}+C T\left\|{ }^{t} L \phi\right\|_{2} \leq C T \sup \left|b_{x}\right|\|\phi\|_{2}+C T\left\|{ }^{t} L \phi\right\|_{2} \\
\therefore\|\phi\|_{2}\left(1-C T \sup \left|b_{x}\right|\right) \leq C T\left\|{ }^{t} L \phi\right\|_{2} .
\end{gathered}
$$

Escolhendo $T_{0}^{\prime}>0$ suficientemente pequeno para que $C T_{0}^{\prime} \sup \left|b_{x}\right|<1$, poderemos dizer que, para qualquer $0<T<T_{0}^{\prime}$,

$$
\|\phi\|_{2} \leq \frac{C}{1-C T_{0}^{\prime}} T\left\|^{t} L \phi\right\|_{2} .
$$

Assim, basta demonstrar este teorema para $L$ ao invés de ${ }^{t} L$. Vamos fazer isto agora.

Sejam $\psi^{+}+\psi_{0}+\psi^{-} \equiv 1$ uma partição $\mathcal{C}^{\infty}$ da unidade em $\mathbb{R}$ com supp $\psi^{-} \subset$ $(-\infty, 0), \operatorname{supp} \psi_{0} \subset(-1,1)$ e $\operatorname{supp} \psi^{+} \subset(0, \infty)$. Para as funções $\phi(x, t)$ no plano, denotaremos por $\widehat{\phi}(\xi, t)$ a transformada de Fourier parcial em relação à variável $x$.

Seja $P^{+}$o operador dado por

$$
P^{+} f(x, t)=\frac{1}{2 \pi} \int_{-\infty}^{\infty} \mathrm{e}^{i x \xi} \psi^{+}(\xi) \hat{f}(\xi, t) \mathrm{d} \xi .
$$

Seja $B(x, t)=\int_{0}^{t} b(x, \tau) \mathrm{d} \tau$. Defina o operador

$$
\begin{aligned}
K^{+} f(x, t) & =\frac{1}{2 \pi} \int_{\mathbb{R}} \int_{T(x)}^{t} \mathrm{e}^{i x \xi+(B(x, t)-B(x, s)) \xi} \tilde{\psi}^{+}(\xi) \hat{f}(\xi, s) \mathrm{d} s \mathrm{~d} \xi \\
& =\int_{T(x)}^{t} \int_{\mathbb{R}} \mathrm{e}^{i x \xi+(B(x, t)-B(x, s))|\xi|} \widehat{\tilde{P}^{+}} f(\xi, s) \frac{\mathrm{d} \xi}{2 \pi} \mathrm{d} s .
\end{aligned}
$$

onde $T(x)$ é $T$ se $b(x, t) \geq 0$ para todo $t$ e $-T$ no caso contrário (lembrando que estamos supondo que a condição $(\mathcal{P})$ vale em todo o plano).

A função $0 \leq \tilde{\psi}^{+} \leq 1$ é escolhida em $\mathcal{C}^{\infty}(\mathbb{R})$ de forma que ela valha 1 no suporte de $\psi^{+}$mas seu suporte ainda esteja contido em $(0, \infty)$. Definimos o operador $\tilde{P}^{+}$de forma análoga a $P^{+}$. Assim, teremos $\tilde{P}^{+} P^{+} f(x, t)=$ $P^{+} f(x, t)$ (enquanto geralmente não é verdade que $P^{+} P^{+} f=P^{+} f$ ).

Para cada $y \geq 0$, vamos denotar por $\mathrm{e}^{-y\left|D_{x}\right|}$ o operador dado por

$$
\mathrm{e}^{-y\left|D_{x}\right|} f(x, t)=\frac{1}{2 \pi} \int_{-\infty}^{\infty} \mathrm{e}^{i x \xi} \mathrm{e}^{-y|\xi|} \hat{f}(\xi, t) \mathrm{d} \xi
$$

Se $y>0$, seja $P_{y}$ o núcleo de Poisson no semiplano,

$$
P_{y}(x)=\frac{1}{\pi} \frac{y}{y^{2}+x^{2}}
$$


cuja transformada de Fourier é e $\mathrm{e}^{-y|\xi|}$ (ver a subseção 3.2.3). Com isso, temos

$$
\mathrm{e}^{-y\left|D_{x}\right|} f(x, t)= \begin{cases}f(x, t), & \text { se } y=0 \\ f * P_{y}(x, t), & \text { se } y \neq 0\end{cases}
$$

Isto implica (proposição 3.2) que $\left|\mathrm{e}^{-y\left|D_{x}\right|} f(x, t)\right| \leq \mathrm{H} f(x, t)$, onde

$$
\mathrm{H} f(x, t)=\sup _{r>0} \frac{1}{2 r} \int_{x-r}^{x+r}|f(s, t)| \mathrm{d} s
$$

é a função maximal de Hardy-Littlewood de $f(x, t)$ em relação a $x$. Sabemos (ver Stein [8], capítulo I para uma demonstração) que existe $C>0$ tal que $\|\mathrm{H} \phi\|_{2} \leq C\|\phi\|_{2}$ para qualquer $\phi \in L^{2}(\mathbb{R})$.

Com isto, (4.20) pode ser reescrita como

$$
K^{+} f(x, t)=\int_{T(x)}^{t} \mathrm{e}^{(B(x, t)-B(x, s))\left|D_{x}\right|} \tilde{P}^{+} f(x, s) \mathrm{d} s .
$$

Vamos agora limitar a norma de $K^{+} f(\cdot, t)$ em $L^{2}(\mathbb{R})$ :

$$
\begin{gathered}
\left\|K^{+} f(\cdot, t)\right\|_{L^{2}(\mathbb{R})}^{2}=\int_{-\infty}^{\infty}\left|K^{+} f(x, t)\right|^{2} \mathrm{~d} x \\
=\int_{-\infty}^{\infty}\left|\int_{-T(x)}^{t} \mathrm{e}^{(B(x, t)-B(x, s))\left|D_{x}\right|} \tilde{P}^{+} f(x, s) \mathrm{d} s\right|^{2} \mathrm{~d} x \\
\leq \int_{-\infty}^{\infty}\left(\int_{-T(x)}^{t}\left|\mathrm{e}^{(B(x, t)-B(x, s))\left|D_{x}\right|} \tilde{P}^{+} f(x, s)\right| \mathrm{d} s\right)^{2} \mathrm{~d} x \\
\leq \int_{-\infty}^{\infty}\left(\int_{-T}^{T}\left|\mathrm{e}^{(B(x, t)-B(x, s))\left|D_{x}\right|} \tilde{P}^{+} f(x, s)\right| \mathrm{d} s\right)^{2} \mathrm{~d} x \\
\leq \int_{-\infty}^{\infty}\left(\int_{-T}^{T} \mathrm{H} \tilde{P}^{+} f(x, s) \mathrm{d} s\right)^{2} \mathrm{~d} x=\left\|\int_{-T}^{T} \mathrm{H} \tilde{P}^{+} f(\cdot, s) \mathrm{d} s\right\|_{2}^{2} \\
\qquad \int_{-T}^{T} \mathrm{H} \tilde{P}^{+} f(\cdot, s) \mathrm{d} s\left\|_{2} \leq \int_{-T}^{T}\right\| \mathrm{H} \tilde{P}^{+} f(\cdot, s) \|_{2} \mathrm{~d} s \quad(\mathrm{des} \text { de Minkowsky) } \\
\leq C \int_{-T}^{T}\left\|\tilde{P}^{+} f(\cdot, s)\right\|_{2} \mathrm{~d} s \\
\quad \leq C \int_{-T}^{T}\|f(\cdot, s)\|_{2} \mathrm{~d} s \\
\therefore\left\|K^{+} f(\cdot, t)\right\|_{2} \leq C \int_{-T}^{T}\|f(\cdot, s)\|_{2} \mathrm{~d} s .
\end{gathered}
$$


Usamos agora a desigualdade de Cauchy-Schwarz:

$$
\begin{aligned}
\left\|K^{+} f(\cdot, t)\right\|_{2} \leq C \mid \int_{-T}^{T}(1)\left(\|f(\cdot, s)\|_{2}\right) & \mathrm{d} s \mid \\
& \leq C \sqrt{2 T}\left\|\left(\|f(\cdot, s)\|_{2}\right)\right\|_{L^{2}(-T, T)[s]}
\end{aligned}
$$

Elevando ao quadrado:

$$
\begin{aligned}
\left\|K^{+} f(\cdot, t)\right\|_{2}^{2} \leq & 2 C^{2} T \int_{-T}^{T}\|f(\cdot, s)\|_{2}^{2} \mathrm{~d} s \\
& =2 C^{2} T \int_{-T}^{T}\left(\int_{\mathbb{R}} f(x, s) \mathrm{d} x\right) \mathrm{d} s=2 C^{2} T\|f\|_{L^{2}(\mathbb{R} \times(-T, T))}
\end{aligned}
$$

Integrando de $-T$ a $T$, obtemos a estimativa de $K^{+} f$ em $L^{2}(\mathbb{R} \times(-T, T))$.

$$
\begin{aligned}
\left\|K^{+} f\right\|_{L^{2}(\mathbb{R} \times(-T, T))}^{2} & \leq 4 C^{2} T^{2}\|f\|_{L^{2}(\mathbb{R} \times(-T, T))} \\
\therefore\left\|K^{+} f\right\|_{L^{2}(\mathbb{R} \times(-T, T))} & \leq 2 C T\|f\|_{L^{2}(\mathbb{R} \times(-T, T))} .
\end{aligned}
$$

Em seguida, vamos examinar o efeito de $K^{+}$em $L \phi, \phi \in \mathcal{C}_{c}^{\infty}(\mathbb{R} \times(-T, T))$. Calculemos $K^{+}\left(\phi_{t}^{+}(x, t)\right)$, onde $\phi^{+}(x, t)=P^{+} \phi(x, t)$ e $\phi_{t}^{+}(x, t)$ é a notação para $P^{+}\left(\partial_{t} \phi(x, t)\right)$ (note que $\partial_{t}$ comuta com a transformada parcial ^ e com os operadores $\left.P^{+}, \tilde{P}^{+}\right)$

Por (4.20),

$$
\begin{aligned}
K^{+} \phi_{t}^{+}(x, t)=\frac{1}{2 \pi} \int_{\mathbb{R}} \int_{T(x)}^{t} & \mathrm{e}^{i x \xi+(B(x, t)-B(x, s)) \xi} \tilde{\psi}^{+}(\xi) \widehat{\phi_{t}^{+}}(\xi, s) \mathrm{d} s \mathrm{~d} \xi \\
= & \frac{1}{2 \pi} \int_{\mathbb{R}} \int_{T(x)}^{t} \mathrm{e}^{i x \xi+(B(x, t)-B(x, s)) \xi} \frac{\partial}{\partial s} \widehat{\phi^{+}}(\xi, s) \mathrm{d} s \mathrm{~d} \xi
\end{aligned}
$$

onde estamos usando que $\tilde{\psi}^{+}(\xi) \psi^{+}(\xi)=\psi^{+}(\xi)$.

A integral interior é:

$$
\begin{aligned}
\int_{T(x)}^{t} \mathrm{e}^{i x \xi+(B(x, t)-B(x, s)) \xi} \frac{\partial}{\partial s} \widehat{\phi^{+}}(\xi, s) \mathrm{d} s=\left.\mathrm{e}^{i x \xi+(B(x, t)-B(x, s)) \xi} \widehat{\phi^{+}}(\xi, s)\right|_{T(x)} ^{t} \\
-\int_{T(x)}^{t}-b(x, s) \xi \mathrm{e}^{i x \xi+(B(x, t)-B(x, s)) \xi} \widehat{\phi^{+}}(\xi, s) \mathrm{d} s \\
=\mathrm{e}^{i x \xi} \widehat{\phi^{+}}(\xi, t)-i \int_{T(x)}^{t} b(x, s) \mathrm{e}^{i \xi+(B(x, t)-B(x, s)) \xi} \widehat{\phi_{x}^{+}}(\xi, s) \mathrm{d} s .
\end{aligned}
$$


Portanto,

$$
\begin{aligned}
& K^{+} \phi_{t}^{+}(x, t)= \\
& \frac{1}{2 \pi} \int_{\mathbb{R}} \mathrm{e}^{i x \xi} \widehat{\phi^{+}}(\xi, t) \mathrm{d} \xi-\frac{i}{2 \pi} \int_{\mathbb{R}} \int_{T(x)}^{t} b(x, s) \mathrm{e}^{i \xi+(B(x, t)-B(x, s)) \xi} \widehat{\phi_{x}^{+}}(\xi, s) \mathrm{d} s \mathrm{~d} \xi \\
& =\phi^{+}(x, t)-i \int_{T(x)}^{t} \int_{\mathbb{R}} b(x, s) \mathrm{e}^{i \xi+(B(x, t)-B(x, s)) \xi} \widehat{\phi_{x}^{+}}(\xi, s) \frac{\mathrm{d} \xi}{2 \pi} \mathrm{d} s \\
& =\phi^{+}(x, t)-i \int_{T(x)}^{t} b(x, s) \mathrm{e}^{(B(x, t)-B(x, s))\left|D_{x}\right|}\left(\phi_{x}^{+}\right)(x, s) \mathrm{d} s .
\end{aligned}
$$

Podemos escrever

$$
\begin{array}{r}
b(x, s) \mathrm{e}^{(B(x, t)-B(x, s))\left|D_{x}\right|}\left(\phi_{x}^{+}\right)(x, s)=\mathrm{e}^{(B(x, t)-B(x, s))\left|D_{x}\right|}\left(b \phi_{x}^{+}\right)(x, s) \\
+\left[b, \mathrm{e}^{\left(B(x, t)-B(x, s)\left|D_{x}\right|\right.}\right] \phi_{x}^{+}(x, s),
\end{array}
$$

onde o colchete entre dois operadores denota o comutador $[X, Y] f=X Y f-$ $Y X f$ e $b$ é o operador de multiplicação $(b f(x, t)=b(x, t) f(x, t))$.

Assim,

$$
\begin{aligned}
& K^{+} \phi_{t}^{+}(x, t)= \\
& \phi^{+}(x, t)-i K^{+}\left(b \phi_{x}^{+}\right)(x, t) \underbrace{-i \int_{T(x)}^{t}\left[b, \mathrm{e}^{(B(x, t)-B(x, s))\left|D_{x}\right|}\right] \phi_{x}^{+}(x, s) \mathrm{d} s}_{R^{+} \phi^{+}(x, t)} .
\end{aligned}
$$

Portanto, lembrando que $L \phi^{+}=\phi_{t}^{+}+i b \phi_{x}^{+}$,

$$
K^{+} L \phi^{+}(x, t)=\phi^{+}(x, t)+R^{+} \phi^{+}(x, t)
$$

Para estimar a norma do resto $R^{+} \phi^{+}(x, t)$, vamos precisar do seguinte resultado:

Afirmação: Dados $f \in \mathcal{S}(\mathbb{R})$ e $h \in \mathcal{C}_{c}^{\infty}(\mathbb{R})$, existe constante $C^{\prime}>0$, independente de $f$ e de $h$, tal que

$$
\forall \epsilon>0, \quad\left|\left[h, \mathrm{e}^{-\epsilon\left|D_{x}\right|}\right] f^{\prime}(x)\right| \leq C^{\prime}\left(\sup \left|h^{\prime}\right|\right) \mathrm{H} f(x) .
$$


De fato,

$$
\begin{gathered}
{\left[h, \mathrm{e}^{-\epsilon\left|D_{x}\right|}\right] f^{\prime}=h(x) P_{\epsilon} * f^{\prime}(x)-P_{\epsilon} *\left(h f^{\prime}\right)} \\
=\int_{\mathbb{R}} h(x) f^{\prime}(x-y) P_{\epsilon}(y) \mathrm{d} y-\int_{\mathbb{R}} h(x-y) f^{\prime}(x-y) P_{\epsilon}(y) \mathrm{d} y \\
=\int_{\mathbb{R}}(h(x)-h(x-y)) f^{\prime}(x-y) P_{\epsilon}(y) \mathrm{d} y \\
=\int_{\mathbb{R}}(h(x)-h(y)) f^{\prime}(y) P_{\epsilon}(x-y) \mathrm{d} y \\
=\underbrace{\left.f(y) P_{\epsilon}(x-y)(h(x)-h(y))\right|_{-\infty} ^{\infty}}_{=0} \quad \text { integração por partes) } \\
-\int_{\mathbb{R}}\left(-\left(P_{\epsilon}\right)^{\prime}(x-y)(h(x)-h(y))+P_{\epsilon}(x-y)\left(-h^{\prime}(y)\right) f(y) \mathrm{d} y\right. \\
=P_{\epsilon} * f h^{\prime}(x)+\left(P_{\epsilon}\right)^{\prime} *((h(x)-h) f)(x) .
\end{gathered}
$$

O primeiro termo é limitado (proposição 3.2) por $\mathrm{H}\left(f h^{\prime}\right)(x)$, que é menor ou igual a $\left(\sup \left|h^{\prime}\right|\right) \mathrm{H} f(x)$. Quanto ao segundo termo, note que

$$
\left(P_{\epsilon}\right)^{\prime}(x)=\frac{\mathrm{d}}{\mathrm{d} x} \frac{1}{\epsilon} P(x / \epsilon)=\frac{1}{\epsilon^{2}} P^{\prime}(x / \epsilon) .
$$

Fazendo $Q(x)=x P^{\prime}(x)$, teremos

$$
Q_{\epsilon}(x)=\frac{1}{\epsilon^{2}} x P^{\prime}(x / \epsilon)=x\left(P_{\epsilon}\right)^{\prime}(x)
$$

e, assim, o segundo termo é igual a

$$
\int_{\mathbb{R}}(h(x)-h(y)) f(y) \frac{Q_{\epsilon}(x-y)}{x-y} \mathrm{~d} y=\left(Q_{\epsilon} * g_{x}\right)(x),
$$

onde $g_{x}$ é a função definida por

$$
g_{x}(y)=\frac{h(x)-h(y)}{x-y} f(y)
$$

Pelo teorema do valor médio, $\left|g_{x}\right| \leq\left(\sup \left|h^{\prime}\right|\right)|f|$. Como $Q$ tem majorante radial integrável, $\left(Q_{\epsilon} * g_{x}\right)(x)$ é limitado por $I\left(\sup \left|h^{\prime}\right|\right) \mathrm{H} f(x)$, onde $I$ é a integral do majorante radial de $Q$. Tomando-se $C^{\prime}=1+I$, a afirmação fica provada. 
Com isto, podemos estimar a norma de $R^{+}$procedendo analogamente ao que foi feito para estimar $K^{+}$. Fazendo $C^{\prime \prime}=C^{\prime} \sup _{\mathbb{R}^{2}}\left|h_{x}\right|$,

$$
\begin{array}{r}
\left\|R^{+} \phi^{+}(\cdot, t)\right\|_{2} \leq C^{\prime \prime} \int_{-T}^{T}\left\|\mathrm{H}\left(\phi^{+}(\cdot, s)\right)\right\|_{2} \mathrm{~d} s \leq C C^{\prime \prime} \int_{-T}^{T}\left\|\phi^{+}(\cdot, s)\right\|_{2} \mathrm{~d} s \\
\leq C C^{\prime \prime} \sqrt{2 T}\left(\int_{\mathbb{R}}\left\|\phi^{+}(\cdot, s)\right\|_{2}^{2} \mathrm{~d} s\right)^{\frac{1}{2}}=C_{3} \sqrt{2 T}\left\|\phi^{+}\right\|_{L^{2}(\mathbb{R} \times(-T, T))},
\end{array}
$$

onde $C_{3}=C C^{\prime \prime}$.

$$
\begin{aligned}
\therefore\left\|R^{+} \phi^{+}\right\|_{L^{2}(\mathbb{R} \times(-T, T))} & =\left(\int_{-T}^{T}\left\|R^{+} \phi^{+}(\cdot, s)\right\|_{2}^{2} \mathrm{~d} s\right)^{\frac{1}{2}} \\
& \leq 2 C_{3} T\left\|\phi^{+}\right\|_{L^{2}(\mathbb{R} \times(-T, T)}
\end{aligned}
$$

Juntando (4.22), (4.23) e (4.24), temos

$$
\begin{aligned}
\left\|\phi^{+}\right\|_{L^{2}(\mathbb{R} \times(-T, T))}= & \left\|K^{+} L \phi^{+}-R^{+} \phi^{+}\right\|_{L^{2}(\mathbb{R} \times(-T, T)} \\
& \leq 2 C T\left\|L \phi^{+}\right\|_{L^{2}(\mathbb{R} \times(-T, T))}+2 C_{3} T\left\|\phi^{+}\right\|_{L^{2}(\mathbb{R} \times(-T, T))} .
\end{aligned}
$$

Sabemos que $\left\|\phi^{+}\right\|_{L^{2}(\mathbb{R} \times(-T, T))} \leq\|\phi\|_{L^{2}(\mathbb{R} \times(-T, T))}$, o que pode ser visto usando-se o teorema de Plancherel e da desigualdade Cauchy-Scwharz. Para estimar $\left\|L \phi^{+}\right\|_{L^{2}(\mathbb{R} \times(-T, T))}$ em função da norma de $L \phi$, vamos escrever

$$
L \phi^{+}=L P^{+} \phi=\left[L, P^{+}\right] \phi+P^{+} L \phi .
$$

Como a derivada $\partial_{t}$ comuta com $P^{+},\left[L, P^{+}\right]=\left[i b \partial_{x}, P^{+}\right]$, que é limitado em $L^{2}$ (ver Stein [9], página 309). Assim, poderemos concluir que existe $C_{4}>0$ independente de $\phi$, tal que

$$
\left\|\phi^{+}\right\|_{L^{2}(\mathbb{R} \times(-T, T))} \leq C_{4} T\|L \phi\|_{L^{2}(\mathbb{R} \times(-T, T))}+C_{4} T\|\phi\|_{L^{2}(\mathbb{R} \times(-T, T))} .
$$

Procedendo analogamente, existe $C_{5}>0$ independente de $\phi$ tal que

$$
\left\|\phi^{-}\right\|_{L^{2}(\mathbb{R} \times(-T, T))} \leq C_{5} T\|L \phi\|_{L^{2}(\mathbb{R} \times(-T, T))}+C_{5} T\|\phi\|_{L^{2}(\mathbb{R} \times(-T, T))} .
$$

Para estimar $\phi_{0}$, definimos $P_{0}$ e $\tilde{P}_{0}$ análogos a $P^{+}$e $\tilde{P}^{+}$(mas usando $\psi_{0}$ ao invés de $\psi^{+}$) e

$$
\begin{aligned}
K_{0} f(x, t) & =\int_{-T}^{t} \int_{\mathbb{R}} \mathrm{e}^{i x \xi+(B(x, t)-B(x, s)) \xi} \widehat{\tilde{P}_{0} f}(\xi, s) \frac{\mathrm{d} \xi}{2 \pi} \mathrm{d} s \\
& =\int_{-T}^{t} \int_{-1}^{1} \mathrm{e}^{i x \xi+(B(x, t)-B(x, s)) \xi} \widehat{\tilde{P}_{0} f}(\xi, s) \frac{\mathrm{d} \xi}{2 \pi} \mathrm{d} s .
\end{aligned}
$$


Seja $\theta \in \mathcal{C}_{c}^{\infty}(\mathbb{R})$ uma função que vale 1 no intervalo $(-2 \eta, 2 \eta)$, onde $\eta>\sup _{\mathbb{R}^{2}}|B|$.

Com isso, $\theta((B(x, t)-B(x, s)) \xi)=1$ sempre que $\xi \in(-1,1)$. Isto nos permite escrever a fórmula de $K_{0}$ como:

$$
\begin{aligned}
K_{0} f(x, t) & =\int_{-T}^{t} \int_{-1}^{1} \mathrm{e}^{i x \xi} \theta((B(x, t)-B(x, s)) \xi) \mathrm{e}^{(B(x, t)-B(x, s)) \xi} \widehat{\tilde{P}_{0} f}(x, s) \frac{\mathrm{d} \xi}{2 \pi} \mathrm{d} s \\
& =\int_{-T}^{t} \int_{\mathbb{R}} \mathrm{e}^{i x \xi} \theta((B(x, t)-B(x, s)) \xi) \mathrm{e}^{(B(x, t)-B(x, s)) \xi} \widehat{\tilde{P}_{0} f}(x, s) \frac{\mathrm{d} \xi}{2 \pi} \mathrm{d} s \\
& =\int_{-T}^{t}\left(\theta\left((B(x, t)-B(x, s)) D_{x}\right) \mathrm{e}^{(B(x, t)-B(x, s)) D_{x}}\right) \tilde{P}_{0} f(x, s) \mathrm{d} s,
\end{aligned}
$$

onde, para cada $y \in \mathbb{R}$, o operador $\theta\left(y D_{x}\right) \mathrm{e}^{y D_{x}}$ é definido pela fórmula

$$
\theta\left(y D_{x}\right) \mathrm{e}^{y D_{x}} f(x, t)=\frac{1}{2 \pi} \int_{\mathbb{R}} \mathrm{e}^{i x \xi} \theta(y \xi) \mathrm{e}^{y \xi} \hat{f}(\xi, t) \mathrm{d} \xi
$$

Seja $G$ a transformada de Fourier inversa de $\theta(\xi) \mathrm{e}^{\xi}$. Dado $y \in \mathbb{R}$ diferente de zero, defina

$$
G_{y}(x)=\frac{1}{|y|} G(x / y)=\mathcal{F}_{\xi}^{-1}\left(\theta(y \xi) \mathrm{e}^{y \xi}\right)(x)
$$

Isto nos dá que

$$
\theta\left(y D_{x}\right) \mathrm{e}^{y D_{x}} f(x, t)= \begin{cases}f * G_{y}(x, t), & \text { se } y \neq 0 \\ f(x, t), & \text { se } y=0\end{cases}
$$

Podemos limitar $\theta\left(y D_{x}\right) \mathrm{e}^{y D_{x}}$ pelo maximal $\mathrm{H} f(x, t)$ multiplicado por uma constante (pois $G$ tem majorante radial limitado). Assim, $G$ é análogo ao núcleo de Poisson no semiplano usado na estimativa de $K^{+}$.

Isto nos permite mostrar, de forma análoga à com que fizemos a estimativa de $K^{+}$, que existe constante $C_{6}>0$ tal que

$$
\left\|\phi_{0}\right\|_{L^{2}(\mathbb{R} \times(-T, T))} \leq C_{6} T\|L \phi\|_{L^{2}(\mathbb{R} \times(-T, T))}+C_{6} T\|\phi\|_{L^{2}(\mathbb{R} \times(-T, T))} .
$$

Assim, como $\phi=\phi^{+}+\phi_{0}+\phi_{-}$, existe constante $C_{7}>0$ tal que

$$
\begin{gathered}
\|\phi\|_{L^{2}(\mathbb{R} \times(-T, T))} \leq C_{7} T\|L \phi\|_{L^{2}(\mathbb{R} \times(-T, T))}+C_{7} T\|\phi\|_{L^{2}(\mathbb{R} \times(-T, T))} . \\
\therefore\|\phi\|_{L^{2}(\mathbb{R} \times(-T, T))} \leq \frac{C_{7} T}{1-C_{7} T}\|L \phi\|_{L^{2}(\mathbb{R} \times(-T, T))} .
\end{gathered}
$$

Tomando $T_{0}<\frac{1}{C}{ }_{7}$ e renomeando $C=C_{7} /\left(1-C_{7} T_{0}\right)$, o teorema está provado. 
Corolário 4.25. Se o campo $L$ da definição 4.8 satisfaz a condição $(\mathcal{P})$ em uma vizinhança da origem, então existem $T_{0}>0$ e $C>0$ tais que, para todo $0<T<T_{0}$, dada $f \in L^{2}\left(\mathbb{R}^{2}\right)$, existe $u \in L^{2}\left(\mathbb{R}^{2}\right)$ com norma

$$
\|u\|_{2} \leq C T\|f\|_{2}
$$

tais que

$$
L u=f \quad e m \mathbb{R} \times(-T, T) \text { (no sentido de distribuição). }
$$

Demonstração. Seja $\Omega_{T}=\mathbb{R} \times(-T, T)$. Considere o funcional linear

$$
\begin{aligned}
\lambda:{ }^{t} L\left(C_{c}^{\infty}\left(\Omega_{T}\right)\right) & \longrightarrow \mathbb{C} \\
{ }^{t} L \phi & \longmapsto \int_{\mathbb{R}^{2}} f(x, t) \phi(x, t) \mathrm{d} x \mathrm{~d} t,
\end{aligned}
$$

que é bem definido, pois o teorema 4.19 implica que ${ }^{t} L$ é injetiva. Pela desigualdade Cauchy-Schwarz e pelo teorema 4.19,

$$
\left|\lambda\left({ }^{t} L \phi\right)\right| \leq\|f\|_{2}\|\phi\|_{2} \leq C T\|f\|_{2}\left\|{ }^{t} L \phi\right\|_{2},
$$

ou seja, $\lambda$ é um funcional linear limitado no subespaço vetorial ${ }^{t} L\left(\mathcal{C}_{c}^{\infty}\left(\Omega_{T}\right)\right)$ de $L^{2}\left(\mathbb{R}^{2}\right)$, com norma $\leq C T\|f\|_{2}$. Pelo teorema de Hahn-Banach, podemos estender $\lambda$ para um funcional linear limitado (e de mesma norma) $\lambda^{*} \mathrm{em}$ $L^{2}\left(\mathbb{R}^{2}\right)$. Pelo teorema da representação de Riesz, existe $u \in L^{2}\left(\mathbb{R}^{2}\right)$, com $\|u\|_{2} \leq C T\|f\|_{2}$ tal que

$$
\int_{\mathbb{R}^{2}} u \phi=\lambda^{*}(\phi), \quad \forall \phi \in L^{2}\left(\mathbb{R}^{2}\right)
$$

Em particular, se $\phi \in \mathcal{C}_{c}^{\infty}\left(\Omega_{T}\right)$,

$$
\langle L u, \phi\rangle=\left\langle u,{ }^{t} L \phi\right\rangle=\lambda\left({ }^{t} L \phi\right)=\langle f, \phi\rangle,
$$

o que significa que $L u=f$ em $\Omega_{T}$ no sentido das distribuições. 


\section{Referências Bibliográficas}

[1] Shiferaw Berhanu, Paulo D. Cordaro, and Jorge Hounie, An Introduction to Involutive Structures, Cambridge University Press, Cambridge, 2008.

[2] Lars Hörmander, The Analysis of Linear Partial Differential Operators. I: Distribution Theory and Fourier Analysis, Springer-Verlag, Berlin, 1983.

[3] Elon Lages Lima, Análise no Espaço $\mathbb{R}^{n}$, Instituto Nacional de Matemática Pura e Aplicada, 2007.

[4] Claude Lobry, Contrôlabilité des systèmes non linéaires, SIAM J. Control 8 (1970), 573-605.

[5] _ Erratum: Contrôlabilité des systèmes non linéaires, SIAM J. Control and Optimization 14 (1976), 387.

[6] L. Nirenberg and F. Treves, Solvability of a First Order Linear Partial Differential Equation, Communications on Pure and Applied Mathematics XVI (1963), 331-351.

[7] Jorge Sotomayor Tello, Lições de Equações Diferenciais Ordinárias, Instituto de Matemática Pura e Aplicada - CNPq, 1979.

[8] Elias M. Stein, Singular Integrals and Differentiability Properties of Functions, Princeton University Press, 1970.

[9] _ Harmonic Analysis: Real-Variable Methods, Orthogonality, and Oscillatory Integrals, Princeton University Press, Princeton, New Jersey, 1993.

[10] Héctor J. Sussmann, Orbits of Families of Vector Fields and Integrability of Distributions, Transactions of the American Mathematical Society 180 (June 1973), 171-188. 